Accepted for publication in The Astrophysical Journal

\title{
A Survey of Chromospheric Activity in the Solar-Type Stars in the Open Cluster M67 ${ }^{1}$
}

\author{
Mark S. Giampapa ${ }^{2}$ \\ giampapa@noao.edu \\ Jeffrey C. Hall ${ }^{3}$ \\ jch@lowell.edu \\ Richard R. Radick ${ }^{4}$ \\ radick@nso.edu \\ and \\ Sallie L. Baliunas ${ }^{5}$ \\ baliunas@cfa.harvard.edu
}

\begin{abstract}
${ }^{1}$ The results presented herein are based on data obtained at the WIYN telescope and at the McMathPierce Solar Telescope, respectively. The WIYN Observatory is a joint facility of the University of WisconsinMadison, Indiana University, Yale University, and the National Optical Astronomy Observatory. The McMath-Pierce Solar Telescope Facility is operated by the National Solar Observatory for the National Science Foundation

${ }^{2}$ National Solar Observatory/NOAO, 950 N. Cherry Ave., POB 26732, Tucson, AZ 85726-6732. The NSO and the NOAO are each operated by the Association of Universities for Research in Astronomy, Inc. (AURA) under cooperative agreement with the National Science Foundation

${ }^{3}$ Lowell Observatory, 1400 W. Mars Hill Rd., Flagstaff, AZ 86001

${ }^{4}$ Air Force Research Laboratory, NSO/Sacramento Peak, Box 62, Sunspot, NM 88349

${ }^{5}$ Harvard-Smithsonian Center for Astrophysics, 60 Garden St., MS 15, Cambridge, MA 02138
\end{abstract}


We present the results of a spectroscopic survey of the Ca II H \& K core strengths in a sample of 60 solar-type stars that are members of the solar-age and solar-metallicity open cluster M67. We adopt the HK index, defined as the summed $\mathrm{H}+\mathrm{K}$ core strengths in $1 \AA$ bandpasses centered on the $\mathrm{H}$ and $\mathrm{K}$ lines, respectively, as a measure of the chromospheric activity that is present. We compare the distribution of mean HK index values for the M67 solar-type stars with the variation of this index as measured for the Sun during the contemporary solar cycle. We find that the stellar distribution in our HK index is broader than that for the solar cycle. Approximately 17\% of the M67 sun-like stars exhibit average HK indices that are less than solar minimum. About 7\%-12\% are characterized by relatively high activity in excess of solar maximum values while $72 \%-80 \%$ of the solar analogs exhibit $\mathrm{Ca}$ II $\mathrm{H}+\mathrm{K}$ strengths within the range of the modern solar cycle. The ranges given reflect uncertainties in the most representative value of the maximum in the HK index to adopt for the solar cycle variations observed during the period A.D. 1976-2004. Thus, 20\%-30\% of our homogeneous sample of sun-like stars have mean chromospheric $\mathrm{H}+\mathrm{K}$ strengths that are outside the range of the contemporary solar cycle. Any cycle-like variability that is present in the M67 solar-type stars appears to be characterized by periods greater than $\sim 6$ years. Finally, we estimate a mean chromospheric age for M67 in the range of 3.8-4.3 Gyr.

Subject headings: stars: solar-type; chromospheres: Ca II H and K emission; open cluster: individual — M67 (NGC 2682); Sun: solar cycle; stellar ages: chromospheric activity

\section{Introduction}

A key objective of investigations in the field of solar-stellar astrophysics is to provide insight on the nature and possible range of solar magnetic field-related atmospheric activity through observations of stellar analogs of the Sun. In this way, we immediately obtain information on the long-term variability of the Sun that otherwise would not be possible (or practically feasible) with, for example, the modern solar data base of high-precision, chromospheric Ca II H and K observations of about three decades (e.g., White \& Livingston 1978; Livingston 1994). This is especially important given that the amplitude of solar and stellar variations in brightness are usually highly correlated with cycle variations in chromospheric emission (Hudson 1988; Radick 1991; Fröhlich 1994; de Toma et al. 2004). Thus, the range of cycle variability in a sample of solar-type stars can be indicative of the 
magnitude of total brightness changes (Zhang et al. 1994) as well as the nature of variability in the spectral irradiance or the particle fluence in the Sun. These forms of solar variability, in turn, may affect global climate change (e.g., Soon et al. 1996; Soon et al. 2000a,b; Foukal 2003; Stott et al. 2003; Haigh 2003, 2005; Soon \& Yaskell 2004 and references therein; Lean et al. 2005).

In addition to the impact on our understanding of the role of the past and future variability of the Sun in global climate, the characteristics of cycle-related variability are a manifestation of the nature of the dynamo mechanism that is operative in the Sun and sunlike stars (Saar \& Baliunas 1992; Saar 2002). Furthermore, the calibration of the empirical correlation between stellar age and chromospheric activity (Walter \& Barry 1991; Soderblom et al. 1991; Simon 1992; Donahue 1998) will be affected by the intrinsic dispersion introduced by the cycle-related variation in chromospheric emission. As an illustration of the magnitude of this effect, if we combine the $25 \%$ range in the Ca II index from minimum to maximum in the solar cycle, as measured by Livingston \& Wallace (2003) from 1975 to 2003, with the exponential decay law proposed by Walter \& Barry (1991) for Ca II then the apparent uncertainty in the age of the Sun arising from its cycle variability would be $\sim 21 \%$, or an age-spread of roughly 4 Gyr - 6 Gyr (see also Baliunas et al. 1998, their Fig. 4).

Recent studies of the comparative properties of the Sun and sun-like stars have emphasized field stars in the solar neighborhood. This approach yields samples that are accessible to detailed and frequent observation with small-to-moderate aperture telescopes, and includes the extensive archive of Ca II H and $\mathrm{K}$ observations from the Mt. Wilson program (Wilson 1978; Vaughan et al. 1978; Baliunas et al. 1995). Baliunas \& Jastrow (1990) utilized data from the Mt. Wilson program to examine the nature and distribution of activity in 74 stars that were selected to be solar-type according to a set of criteria that included age, mean level of chromospheric activity, and mass as inferred from photometric $B-V$ color. They estimated the potential range of solar magnetic activity over time scales of centuries, and the approximate magnitude of the associated changes that would be implied in the solar irradiance, particularly in the regime of exceptionally low-activity that is presumed to have occurred during the Maunder Minimum (White 1978). Among the distinctive features of the tentative results discussed by Baliunas \& Jastrow (1990) were a significant width of the distribution of mean chromospheric activity measured at random phases of centuries-long variability and a possible bimodal distribution of mean chromospheric activity. They inferred from their stellar sample, using a particular method of sampling the time series of observations, that exceptionally quiescent levels of activity occurred at a frequency of about one-third of the time. This estimate, in turn, was primarily associated with four stars in their sample that exhibited little variability (i.e., instrumentally flat time series in the Mt. Wilson S-index of relative Ca II H and K strength) over periods of approximately two decades. 
Baliunas \& Jastrow (1990) suggested that these objects were in the stellar counterpart of the solar Maunder-minimum state. In a subsequent study of a subsample of 10 solar-type stars, Zhang et al. (1994) cautioned that the small number of nearby solar-type stars, and the heterogeneity that is inherent in such field star samples, needed to be addressed.

Hall \& Lockwood (2004) reexamined the conclusions by Baliunas \& Jastrow (1990) using a refined sample of solar counterparts in the field as monitored since 1994 with their SolarStellar Spectrograph (SSS) at Lowell Observatory in Flagstaff, Arizona (Hall \& Lockwood 1995). These investigators also find that one-third of the solar counterparts in their sample do not exhibit cyclic behavior, in agreement with the results of Baliunas \& Jastrow (1990). However, in contrast to the earlier study, the distribution of $\mathrm{H}$ and $\mathrm{K}$ activity in the Hall \& Lockwood (2004) sample is unimodal rather than bimodal. Moreover, these investigators note that the level of chromospheric $\mathrm{H}$ and $\mathrm{K}$ emission in the non-cyclic, flat stars spans a range from levels below that of solar minimum to greater than that seen at solar maximum. Hence, as Hall \& Lockwood conclude, non-cyclic behavior is not necessarily associated with relatively reduced chromospheric activity.

These kinds of studies illustrate the crucial discrepancies in conclusions that can arise from investigations of heterogeneous samples of field stars. For example, in the case of an ostensibly solar-type star that exhibits a low-level of activity, it is particularly critical to know its evolutionary status before concluding that it is a Maunder-minimum candidate. In fact, Wright (2004) claims, on the basis of an analysis of Hipparcos data and a transformation of the Mt. Wilson S-index to a normalized chromospheric emission flux $\left(R_{H K}^{\prime}\right)$, that nearly all the stars thus far identified as Maunder minimum candidates are actually evolved or subgiant stars with intrinsically low (and constant) chromospheric activity rather than solar-type stars that are in a temporary state of quiescence. A further examination of this result based on high resolution $\mathrm{H} \& \mathrm{~K}$ line spectra combined with an independent flux calibration technique (e.g., following the methods of Linsky et al. 1979) is merited.

In this investigation, we extend these pioneering studies to a sample of solar-type cluster members that are homogeneous in age and chemical composition. The open cluster M67 is an especially appropriate target of observation since it is approximately the same age (about 5 Gyr \pm 1 Gyr; Demarque, Guenther \& Green 1992) and of the same metallicity as the Sun (Barry \& Cromwell 1974). Thus, the solar-type members represent analogs of the Sun at random phases in their activity cycles. Therefore, a survey of the "Suns of M67" should reveal the possible range of solar chromospheric activity, thus immediately yielding information on the potential long-term variability of the Sun. We discuss in $\S 2$ the observations and reduction. In $\S 3$, we describe the methodology adopted for the calibration of the stellar spectra to relative intensity and discuss our estimates of the random and systematic errors. 
We present the results in $\S 4$ followed by discussion in $\S 5$. We summarize our findings and indicate the future directions of this program in $\S 6$.

\section{Observations}

We utilized the 3.5-m WIYN telescope in conjunction with the Hydra multi-fiber positioner to perform multi-object spectroscopy over a one-degree field. The Hydra instrument is described by Barden \& Armandroff (1995). We used a bench-mounted spectrograph with the T2KC CCD, Simmons camera, the blue fiber cables (3 arcsec diameter) and the 1200 $g \mathrm{~mm}^{-1}$ grating in second order. $\mathrm{A} \mathrm{CuSO}_{4}$ blocking filter was employed to suppress red leak. This configuration yields a spectral resolution of $0.80 \AA \pm 0.03 \AA$, as measured by the FWHMs of CuAr comparison lines extending from $3875 \AA$ to $3995 \AA$.

The stars are selected from the Girard et al. (1989) proper motion study combined with the CCD photometry given by Montgomery, Marschall \& Janes (1993). For those objects from the Girard et al. study where photometry from Montgomery et al. is not available, we adopt the photometry given by Sanders (1989). We note that Girard et al. determine two values of the membership probability: One is based solely on proper motion while the other value takes into account the position of a star relative to the center of the cluster. This latter membership probability assumes a particular model for the spatial distribution of cluster stars. In general, we include stars with membership probabilities that are $\gtrsim 90 \%$ in one or the other of the probabilities given by Girard et al. (1989). In a few cases, we include objects with membership probabilities that are slightly less than $90 \%$ in both estimates. In these instances, the position of the object in the color-magnitude diagram provides further support for the assumption that the star is a bona-fide cluster member.

Our photometric criteria include stars in the range of apparent brightness of approximately $14 \leq V \lesssim 15$ and intrinsic color of $+0.58 \leq(B-V)_{0} \leq+0.76$, where we adopt a value of $E(B-V)=+0.05$ for the color excess in M67 (Montgomery et al. 1993). The resulting spectral range of the solar-type sample is $\sim \mathrm{F} 9$ - G9, following the $(B-V)$ colorspectral-type calibration given by Johnson (1966). In this way, we can ensure that we are observing stars that are close to the Sun in their photospheric properties while still retaining a relatively large sample size. We show in Fig. 1 a color-magnitude diagram of the cluster with the survey objects indicated in order to illustrate the context of our investigation in the long history of observational studies of the M67 open cluster.

The reduction follows standard procedures as outlined by Massey (1997) for the re-

duction of WIYN Hydra data utilizing iraf routines. In particular, the CCD frames are 
bias-subtracted and divided by a normalized flat field frame obtained by combining a series of dome flats. A dispersion correction is applied with the aid of CuAr comparison lamp spectra acquired before and after each series of integrations for a given field configuration of the Hydra positioner. A correction for scattered light was applied within the iraf dohydra routine. The scattered light in the object frames is generally at the 1-2 ADU level, or a few percent of the level of the dark $\mathrm{H}$ and K cores. Sky subtraction was performed with a mean sky spectrum obtained from the individual sky fibers. A radial velocity correction for the changing component of the Earth's rotation (heliocentric correction) was computed and applied using the iraf routines rvcor and dopcor, respectively.

The results discussed herein are based on spectra obtained during the observing seasons from 1996-2001. The long-term nature of the program enables us to search for variability that, for example, may arise from activity cycles analogous to the $\sim 11$-year solar cycle. In view of the difficulty of obtaining high precision measurements of the deep cores of the $\mathrm{Ca}$ II $\mathrm{H}$ and $\mathrm{K}$ resonance lines in faint stars at moderate-to-high spectral resolutions with moderate-aperture telescopes, we utilize the combined spectra obtained during this period for the purposes of the survey. While this has the effect of averaging intrinsic variations in the Ca II core strength due to chromospheric activity, it nevertheless yields high-precision measurements of the average Ca II strength.

\section{Calibration}

The calibration of the Hydra spectra to residual intensity follows the empirical relations given by Hall \& Lockwood (1995) as functions of $(B-V)$ color. These relations give the residual intensities at pseudocontinuum points at $3912 \AA$ and $4000 \AA$, respectively, each as a function of $(B-V)$ color. Since these calibrations were established using spectra at echelle resolutions, we found it necessary to apply a small correction to the calibrations at the pseudocontinuum points for our somewhat lower-resolution Hydra spectra. In particular, we degraded the calibrated solar spectrum given in the NSO Solar Flux Atlas to the resolution of the Hydra spectra. We then measured the relative intensities at the pseudocontinuum points at $3912 \AA$ and $4000 \AA$, respectively, in the degraded solar spectrum and compared the values with those computed from the relations cited above for the mean $B-V$ color of the Sun of +0.65 (VandenBerg \& Bridges 1984). We adopted the (small) differences as constant corrections applied to the calibrations of our stellar spectra. We display in Fig. 2 a comparison of the NSO solar atlas spectrum in the Ca II H\&K line region with our calibration of the spectrum of a solar-type star observed in M67. The correspondence of the solar and stellar spectra confirm the self-consistent nature of our calibration procedure. 
We adopt the HK index as a measure of the chromospheric activity in the stars in our sample. The HK index is the sum of the relative intensities in $1 \AA$ bandpasses centered at the $\mathrm{H}$ and $\mathrm{K}$ lines in the calibrated Hydra spectra. This definition is analogous to that used by White \& Livingston (1981) in their long-term program of measurement of the full disk $\mathrm{Ca}$ II H and $\mathrm{K}$ line profiles in the integrated solar spectrum.

Our processing of the stellar data includes the use of a high quality lunar spectrum, obtained with the Hydra bench spectrograph in our configuration, as a template. The stellar spectra are shifted in wavelength until the lunar and each stellar spectrum are aligned in the Ca II H \& $\mathrm{K}$ region. We define the central wavelengths for the $\mathrm{H}$ and $\mathrm{K}$ lines by the location of the minima of the $\mathrm{H} \& \mathrm{~K}$ features in the lunar template. In this way, the locations in wavelength of the bandpasses for the computation of the HK index can be established in an objective manner and applied uniformly to all the stellar spectra. Likewise, the locations of the psuedocontinuum points are identified as the maximum values in the observed lunar spectrum to within about one-half a resolution element of $3912 \AA$ amd $4000 \AA$, respectively. The wavelengths of these locations are used to find the corresponding psuedocontinuum points in the stellar spectra for the purposes of normalization and calibration. The sum of the relative intensities in the $\mathrm{H}$ and $\mathrm{K}$ line cores are then computed for the calibrated spectra with the aid of a spline fit to the pixel values within the $1 \AA$ bandpasses, including the interpolated values at the locations of the bandpass boundaries at $\pm 0.5 \AA$ from the fixed central wavelength values.

In a similar manner, the random errors due to photon noise are estimated from the uncalibrated spectra (in ADUs) and taking into account the gain of the CCD. That is, the noise estimate is based on the sum of the estimated photon counts inferred from a combination of the CCD gain and a smooth spline fit to the pixel values within the respective $1 \AA$ bandpasses for the $\mathrm{H}$ and $\mathrm{K}$ lines (including the interpolated bandpass boundaries). In addition, a systematic overestimate of the $\mathrm{H} \& \mathrm{~K}$ fluxes is present in our results due to "smearing" of any core emission as a result of the use of only moderate spectral resolution. In order to estimate the magnitude of this effect, we degraded a high resolution solar spectrum to the $0.8 \AA$ resolution of the Hydra bench spectrograph. We found that the measured HK index increased by $4.7 \%$ relative to the value we measured for the undegraded spectrum. We therefore take this factor into account when comparing the solar data to our M67 data in the following discussion. In particular, we adjust the measured solar HK index values upward by $4.7 \%$ when comparing the solar data to our M67 data so that both data-sets are on the same scale. 


\section{Results}

We show in Table 1 the results for our survey of chromospheric Ca II H+K core strengths in our sample of solar-type stars in M67. The mean HK index and error in the mean (inferred from the quadratic sum of the errors in the individual spectra) are listed for each program star by Sanders number. We also give the de-reddened $B-V$ color, the apparent visual magnitude (from Montgomery et al. 1993), and the membership probabilities (as described above). The final column indicates the binary status of the object according to information kindly provided by R. Mathieu in advance of publication. No entry in this column means that no information is available. We note, parenthetically, that we exclude S1112 from the following analysis and discussion. This object, which exhibits strong $\mathrm{H}$ and $\mathrm{K}$ line emission in our spectra, is a detected X-ray source and a suspected RS CVn binary system (see note to Table 1). In addition, we utilized unassigned fiber cables in the Hydra instrument to obtain spectra of M67 dwarf stars that are just outside the intrinsic $B-V$ color range we adopt as our criterion for solar-type. We provide the results for these stars in Table 1 for their potential utilization in other investigations. However, we do not include these objects in the analysis and discussion given herein.

The results for the remaining 60 stars in Table 1 that meet our photometric criteria given in $\S 2$ for solar-type are encapsulated in the histogram in Fig. 3, which shows the distribution of the values of the HK index. In order to place the stellar observations in context, we also display the distribution of the HK index for the solar cycle as measured from 1976 to 2004 (Livingston 2005, private communication) but adjusted upward by 4.7\% in order to take into account the substantial difference in spectral resolution between the two data-sets, as discussed in $§ 3$. Inspection of Fig. 3 reveals that the distribution of HK index for the solar-type stars in M67 is broader than the range of the contemporary solar cycle. The stellar distribution also appears unimodal and slightly skewed with a tail toward higher activity stars. We note that the solar cycle distribution is slightly skewed toward higher activity levels, as is the distribution of Mt. Wilson S-index values for the sample of field solar-type stars investigated by Hall \& Lockwood (2004; see their Fig. 2). This could arise if the decay from maximum in the individual stellar cycles is longer than the rise time from minimum. The shape of the $\sim 11$-year solar cycle exhibits this same characteristic.

A comparison of the stellar with the solar distribution shows that about $17 \%$ of the solar-type stars in the M67 sample exhibit values of the HK index less than solar minimum; $\sim 7 \%$ are in excess of solar maximum while approximately $77 \%$ exhibit values of the $\mathrm{HK}$ index within the range of the solar cycle. Thus, approximately $24 \%$ of the M67 sun-like stars are characterized by a level of chromospheric activity that is outside the range of the contemporary solar cycle while significant overlap with the range of the solar cycle is present 
in $80 \%$ of the stellar sample. If we exclude the slight extension of the solar data to the unusually high HK index of approximately $230 \mathrm{~m} \AA$ and instead adopt a more representative maximum value of $\mathrm{HK} \approx 225 \mathrm{~m} \AA$ (from inspection of Fig. 3), then the fraction of stars that exceeds the solar activity maximum becomes approximately $12 \%$. The fraction that is within the range of the solar cycle decreases to about $72 \%$.

We examine in Fig. 4 the color dependence of the HK index as a function of intrinsic $B-V$ color. We only note that the scatter in HK index appears to increase toward the lower mass stars in the sample. This kind of increasing dispersion in a magnetic activity diagnostic toward lower mass is a feature of late-type dwarf stars in clusters, at least as seen in $\mathrm{H} \alpha$ (e.g, Stauffer et al. 1991). In order to gain further insight on the contributions to the total M67 distribution in Fig. 3 by color bins, we display a sequence of histograms in Fig. 5 delineated according to subsets in $B-V$ intrinsic color. The histograms in Fig. 5 exhibit considerable overlap with the solar cycle distribution. However, departures from the cycle distribution toward both higher activity levels exceeding solar maximum and levels below solar minimum appear more prevalent toward later spectral types.

It has long been recognized that binary membership can enhance and prolong magnetic field-related activity. We show in Fig. 6 the distribution of the HK index in the M67 sample with the contribution by known binaries separately indicated. The HK index distribution for the binaries exhibits significant overlap with the distribution of the M67 solar-type stars that are presumably single (or for which no radial velocity data are yet available). The binary star distribution in HK strength appears to be well segregated between relatively low and high values. However, we hesitate to attribute any physical significance to this apparent gap given the small sample size. Finally, we give in Fig. 7 a plot of the HK index versus period for the binaries in our sample with known orbital periods, kindly provided in advance of publication by Mathieu (2005, private communication). In contrast to the active RS CVn binary systems in M67 (Belloni, Verbunt \& Mathieu 1998; van den Berg et al. 2004), no

correlation between chromospheric Ca II core strength and orbital period is apparent in the binary stars in our solar-type sample.

\section{Discussion}

The broader distribution in Ca II H+K core strengths in the M67 solar-type stars, compared to that of the Sun during the contemporary epoch, suggests that the potential excursion in the amplitude of the solar cycle is greater than what we have seen so far. The stars with values of the HK index noticeably smaller than that of solar minimum may be in a prolonged state of relative quiescence analogous to the Maunder Minimum of the Sun 
during 1645-1715 C.E. when visible manifestations of solar activity, e.g., sunspot number, substantially decreased for a period much longer than the sunspot cycle. That persistent period of reduced solar activity occurred during the extreme phases (ca. mid-16th through 17th century) of a climatic anomaly characterized by a general cooling and referred to as the Little Ice Age. That anomaly, like the Medieval Warm Period which preceded it, is climatically, spatially and temporally complex, with evidence of regional variations in conditions indicating a sensitivity to a variety of forcings, including that due to a variable Sun (Rind 2002). The plausible assumption of a multi-decadal period of decreased total solar irradiance accompanying the observationally established low levels of solar magnetic activity has been postulated as a contributing factor to the sharply deteriorated conditions of the 17th-century (see, e.g., Eddy 1976; Foukal \& Lean 1990; Hoyt \& Schatten 1997; Soon \& Yaskell 2004). However, quantitative estimates of the presumed decrease in the solar irradiance during this time are uncertain; recent work (Wang, Lean \& Sheeley 2005; Lean et al. 2005) concludes that the secular increase in the total irradiance from the Maunder Minimum to the current cycle minima is less than the initial estimates that appeared in the literature beginning over a decade ago.

The $\sim 17 \%$ fraction of M67 sun-like stars in our sample that is at this relatively quiescent level would represent the frequency at which the Sun enters a Maunder Minimum. This frequency is lower than the estimated frequency that the Sun exhibits especially low levels of magnetic field-related activity. In particular, Damon (1977) inferred from the proxy terrestrial record of solar magnetic activity based on the ${ }^{14} \mathrm{C}$ radioisotope that the Sun has spent about one-third of the time in the past several millennia in magnetic activity minima. Our results for the fraction of M67 solar-type stars that exhibit especially quiescent levels of activity is also lower than the $\sim$ one-third frequency seen in samples consisting of field solar-type stars (Baliunas \& Jastrow 1990; Hall \& Lockwood 2004). However, it is higher than the fraction Wright (2004) finds when subgiants are removed from the Mt. Wilson field star sample.

Our M67 sample of solar-type stars, which is presumably homogeneous in age and metallicity, does not exhibit the bimodal distribution that appeared in the heterogeneous sample of field stars studied by Baliunas \& Jastrow (1990). From a purely qualitative perspective, our results imply that exceptional quiescence, such as that during a Maunder Minimum-like episode, is simply a low-amplitude extension of the solar dynamo rather than a separate dynamo mode. In this regard, Ribes \& Nismes-Ribes (1993) concluded from their analysis of historical observations that the solar cycle persisted through the Maunder Minimum, though at exceptionally low amplitudes in terms of sunspot number. Likewise, the occurrence of activity levels that exceed contemporary solar maximum values could be a manifestation of the excursion to enhanced levels of activity in a single mode of dynamo 
operation. Our results $(\S 4)$ would suggest that exceptionally high levels of activity can occur at a frequency of $\sim 7 \%-12 \%$ of the time.

We present in Fig. 8 another depiction of the comparison between the solar cycle and the M67 solar-type star distributions, respectively, in the form of cumulative probability functions of the HK index. The curve for the M67 sample reflects the broader distribution in HK index compared to the solar cycle. Inspection of Fig. 8 readily reveals that the median values for the M67 and the modern solar cycle distributions, respectively, are nearly identical. Finally, a Spearman rank correlation test applied to the two distributions in Fig. 8 yields a moderately high correlation at $\rho=0.881$. However, if we apply this test after omitting the "supersolar" stars with HK > $300 \mathrm{~m} \AA$ then we find an extremely high correlation at $\rho=$ 0.978. This result, while not conclusive, is consistent with an activity-cycle origin, analogous to the solar cycle, for the observed HK index distribution for the solar-type stars in M67.

The HK index distribution in M67 according to color, as illustrated in Figs. 4-5, merits further consideration. The distribution in Fig. 5(a) appears skewed with a tail toward higher activity stars with $\mathrm{HK} \gtrsim 200 \mathrm{~m} \AA$. A comparison with Fig. 4 reveals that the peak in the distribution consists mainly of bluer stars with intrinsic $B-V$ colors $\lesssim 0.60$. These objects may indeed be relatively more chromospherically active in this color range. However, it is important to recognize that the non-chromospheric, radiative equilibrium contribution to the core flux in the $\mathrm{Ca}$ II H \& $\mathrm{K}$ lines will be relatively higher in hotter stars and decline toward cooler objects (Linsky et al. 1979; Noyes et al. 1984). A reliable calibration of the radiative equilibrium flux for the $1 \AA$ bandpasses that define the HK index has not yet been done.

The distribution in Fig. 5(b) is especially interesting because this subsample is the most photometrically similar to the Sun. In particular, the range of colors that has been quoted in the literature for the Sun is $0.63 \leq B-V \leq 0.67$ (VandenBerg \& Bridges 1984, see their Table 2). Hence, the $21 \mathrm{M} 67$ sun-like stars we observed in this color range are photometric counterparts of the Sun and thus can be considered true solar analogs, following the definition of a solar analog as given by Cayrel de Strobel \& Bentolilla (1989; also see Cayrel de Strobel 1996). In the case of these M67 solar analogs, 2 stars or $10 \%$ are in a state of enhanced activity with HK indices in excess of solar maximum values while 4 stars (about 19\%) exhibit values of HK that are below that of the contemporary solar minimum HK index; $71 \%$, or 15 stars, have an average HK index that is within the range of the modern solar cycle. Thus, $29 \%$ of the solar analogs are characterized by levels of chromospheric activity that are outside the range of the modern solar cycle. In brief summary, a significant fraction of this homogeneous sample of solar analogs exhibits activity that is, in amplitude, consistent with the solar cycle as seen in the chromospheric Ca II resonance lines. The fraction that is 
outside of the cycle range can be indicative of the frequency of excursions in the solar cycle to either enhanced levels of activity or unusually quiescent episodes (e.g., Maunder minima).

Those stars that are slightly cooler and less massive than the Sun in the color range of $0.68 \leq(B-V)_{0} \leq 0.72$ also show substantial overlap with the solar cycle with 6/11 stars in the cycle range; 3-4 stars are more active than solar maximum and 1-2 objects have values of the HK index less than the contemporary solar minimum. Similarly, the coolest stars observed in our sample with intrinsic $B-V$ colors in the range of $0.73-0.76$ include 2 stars more quiescent than solar minimum, no objects more active than solar maximum and 4 stars in the range of the solar cycle. In brief summary, the solar-type stars in this approximately solar-age and solar-metallicity cluster exhibit levels of chromospheric activity, as represented by our HK index, that are substantially within the range of the solar cycle. Excursions outside of the solar cycle range of activity occur at a frequency of about $29 \%$ for the entire solar-type sample, and at frequencies broadly in the range of $\sim 22 \%-45 \%$ for the subsets of the sample given in Fig. 5.

\subsection{Implications for Brightness Changes}

Given the correlation between variations in chromospheric emission and changes in brightness or irradiance, as in the case of the Sun, it is of interest to examine the implications of the range of chromospheric activity we see in M67 for the possible range of brightness amplitude variations that may occur. In their long-term study of the photometric variability of field solar-type stars, Lockwood, Skiff \& Radick (1997; their Fig. 16) present the mean amplitude of variation in photometric brightness as a function of $\log R_{H K}^{\prime}$, i.e., the net chromospheric radiative flux in the $\mathrm{H}+\mathrm{K}$ lines, normalized by the stellar bolometric flux $\left(=\sigma T_{e f f}^{4}\right)$.

In order to estimate the potential range in amplitude of brightness changes in our sample of M67 sun-like stars, we must first estimate the net chromospheric radiative loss rates in the $\mathrm{H} \& \mathrm{~K}$ lines from the HK index and the stellar effective temperature. We utilize the calibrations given by Hall \& Lockwood (1996) as a function of $B-V$ color in order to estimate the effective temperature and to obtain the total surface flux in the $\mathrm{H} \& \mathrm{~K}$ cores from the HK index. An empirical correction for the radiative equilibrium (photospheric) contribution to the $\mathrm{H}$ and $\mathrm{K}$ core flux as a function of $B-V$ color is given by Noyes et al. (1984). This approach yields an estimate of $R_{H K}^{\prime}$.

Using our estimates of this parameter from above, we find a range in $\log R_{H K}^{\prime}$ of $\sim$ -4.35 to -5.1. From the results of Lockwood et al. (1997) this range in log $R_{H K}^{\prime}$ corresponds 
to brightness variations extending from sun-like $(\sim 0.1 \%)$ to nearly $3 \%$, or considerably in excess of that of the contemporary Sun. Whether long-term brightness variability of these amplitudes actually occurs in the M67 solar-type stars will require verification through high-precision photometric monitoring.

\subsection{Variability}

The acquisition of the data for this program over several observing seasons enables us to conduct a preliminary investigation of the nature of long-term variability in the HK index in the solar-type stars in M67. We display in Fig. 9(a)-(n) the variation of the HK index by season in those program objects for which we have measurements in at least two observing seasons. The panels are ordered by decreasing $B-V$ color and span the 1996-2002 seasons. We also include in Fig. 9, for comparative purposes, the annual mean values of the HK index for the Sun during this same period.

Inspection of Fig. 9 reveals the presence of variability in the HK index that exceeds the formal errors in practically all the program objects. The time series are not sufficiently long to enable us to conclusively identify the presence of magnetic activity cycles, analogous to the solar cycle, and to measure actual cycle periods, though some cases are suggestive of a cycle-like variation. It does appear from the results displayed in Fig. 9 that if activity cycles are present then their periods are greater than $\sim 6$ years.

We include in Fig. 10 further examples of comparable portions (in time) of the solar cycle variation based on mean annual HK index values for comparison with the panels in Fig. 9. In general, the variability seen in the M67 solar-type stars appears to be characterized by higher amplitudes. We summarize the seasonal variability in HK index in Table 2 for our program objects. The rms variation in HK index among the M67 solar-type stars ranges from $4.60 \mathrm{~m} \AA$ to $92.1 \mathrm{~m} \AA$ with a mean rms of $19.9 \mathrm{~m} \AA$. We also include in Table 2 the $\mathrm{rms}$ variations for the Sun for the examples given in Fig. 10 as well as for the period from 19762004. In the case of the latter, the rms deviation of the annual mean HK index for the Sun is $7.90 \mathrm{~m} \AA$, which is within the range seen for the M67 solar-type stars though significantly lower than the mean rms for the sample. Thus, we find that, while there are seasonal variations comparable to that seen in the Sun in some M67 solar-type stars, variability in the HK index substantially in excess to that of the Sun dominates our results. We do not see a clear correlation between root mean square deviation and the HK index values in the entire data-set (Fig. 11). However, inspection of Fig. 11 suggests a possible correlation of increasing rms deviation with HK index for values of $\mathrm{rms} \gtrsim 20 \mathrm{~m} \AA$. A more precise study of variability will require a long-term program, ideally with spectra obtained at higher spectral 
resolution in order to accurately assess the nature of the variability in the $\mathrm{H}$ and $\mathrm{K}$ line cores.

We construct in Fig. 12 the histogram of all the HK index values measured seasonally for the solar-type stars. Not surprisingly, the distribution is broader than that in Fig. 3, which is based on the HK index for each star averaged over several seasons. We find in Fig. 12 that $20 \%$ of the HK index measurements are less than the modern solar minimum value while Ca II core strengths in excess of solar maximum occur at a frequency of $11 \%$. Thus, about $31 \%$ of the HK index measurements are outside the range of the solar cycle in our sample of M67 solar-type stars when examined on a seasonal basis.

\subsection{Chromospheric Activity and Stellar Age}

The calibration of the empirical correlation between chromospheric activity and stellar age (Skumanich 1972) can yield an additional tool for the determination of the ages of field stars. It is therefore important to investigate quantitatively the magnitude of the impact of intrinsic variations in chromospheric activity, such as magnetic cycle variability, on the calibration of this relation.

Relations between the net radiative chromospheric flux parameter in the Ca II H \& $\mathrm{K}$ lines, $R_{H K}^{\prime}$, and age have been given by Soderblom et al. (1991) and subsequently refined and extended to younger ages $\lesssim 1$ Gyr by Donahue (1993; also see Donahue 1998). We adopt this age-activity calibration to examine the apparent age spread implied by the dispersion in chromospheric activity in both M67 and the solar cycle. In the case of the solar cycle, we utilize the HK index values based on the actual measurements from the high resolution solar spectra in order to give a more realistic estimate of the solar chromospheric age.

Inserting our estimates of $R_{H K}^{\prime}$ from $\S 5.1$ into the age-activity calibration given by Donahue (1998) yields the results in Fig. 13, shown both for the solar-type stars in M67 and the contemporary solar cycle. The broad extent of the apparent age range due to the cycle variation in Ca II in the Sun is $\sim 2.5$ Gyr to about 6 Gyr. The mean chromospheric age of the Sun is approximately 4.26 Gyr. We note, parenthetically, that Baliunas et al. (1998) similarly estimated the apparent (chromospheric) age, or error in estimating the age of the Sun over the last four hundred years if chromospheric activity were sampled at a random phase of long-term variability, namely, 3 to 8 Gyr at the extremes with presumably a narrower range in apparent age for most of the period. The age range among the M67 solar-type stars implied by the range of observed chromospheric activity is even broader, extending from less than 1 Gyr to about 7.5 Gyr. The mean chromospheric age inferred from our sample of solar-type stars is approximately 3.75 Gyr while the median age for the 
M67 distribution is about 3.33 Gyr. Taking into account the possibility of a systematic bias in our stellar HK index values (§3) yields a mean chromospheric age of 4.32 Gyr and a median of 3.83 Gyr. In summary, the mean chromospheric age for the solar-type members of M67 considered herein is in the range of roughly 3.8 - 4.3 Gyr while that for the Sun is approximately 4.3 Gyr. Clearly, age determinations based on chromospheric emission can vary considerably, depending for each star on the phase of its magnetic activity cycle at the time of observation and, in the case of cluster members that are presumably homogeneous in age, on the dispersion in rotational velocities that may be present.

\subsection{The Range of Chromospheric Activity in the M67 Solar-Type Stars}

A surprising result of our investigation is the occurrence of solar-type stars that exhibit levels of chromospheric activity in excess of that seen at the maximum of the contemporary solar-activity cycle. An extreme example is illustrated in Fig. 14 where Ca II H and K line core emission is clearly seen in this spectrum of S1452. This object is not known to be a binary at a precision of $\pm 2 \mathrm{~km} \mathrm{~s}^{-1}$ in the possible variation of its radial velocity (R. D. Mathieu, private communication).

An interpretation of our results is that the origin of the dispersion in chromospheric activity among the solar-type stars in M67 is due to the range of amplitudes in their cycle properties. But we also know, in general, that magnetic field-related chromospheric activity increases with increasing equatorial rotational velocity in late-type stars. Thus, those M67 solar-type stars with relatively enhanced levels of activity, compared to the Sun, may be rotating more rapidly, thereby giving rise to stronger dynamo action. If the chromospheric Ca II H and K strength is proportional to the rotational velocity (following Skumanich 1972) then this would suggest for the high-activity M67 stars in Fig. 3-with roughly twice the HK index as the mean Sun-that their rotational velocities are in the $3-4 \mathrm{~km} \mathrm{~s}^{-1}$ range, or slightly more.

Obviously, this issue can only be addressed through measurements of (a) rotation period, via ultra-high precision photometric observations of spot modulation, (b) through spectrophotometric observations of the rotational modulation of the $\mathrm{H}$ and $\mathrm{K}$ lines, or (c) by spectroscopic measurements of the projected rotation velocity. If the results ultimately reveal a significant spread in rotation rates then this would argue against activity cycles as the principal origin of the distribution of activity in Fig. 3. Moreover, it would raise the important question of why the angular momentum histories of the Sun and the M67 solar counterparts differ. If, however, the M67 sun-like stars exhibit rotational velocities that are more solar-like $\left(\sim 2-3 \mathrm{~km} \mathrm{~s}^{-1}\right)$ then this would suggest that activity cycles-similar to the 
modern solar cycle but characterized by significant differences in amplitude-are the origin of the dispersion in chromospheric activity we see in Fig. 3. However, this would then raise the question of why the contemporary Sun is in a relatively subdued state of activity compared to other sun-like stars of solar age and solar metallicity.

\section{Summary and Future Directions}

We summarize in the following our principal findings and discuss our future directions of research based on these results.

We find that the HK index distribution for the solar-type stars in M67 is broader than that of the contemporary solar cycle though with significant overlap. More specifically, $72 \%$ - $87 \%$ of the 60 solar-type stars in our sample exhibit mean HK index measures, averaged over several seasons of observation, that are within the range of the modern solar cycle. The 21 solar analogs we observed in our M67 sample, i.e., stars that are photometric analogs of the Sun, exhibit a similar correspondence to the solar cycle distribution in HK index, with $71 \%$ of these objects characterized by an average HK index within the range of the contemporary solar cycle. The results for the seasonal distribution of HK index are similar in terms of the frequency of values that are within and outside the range of the modern solar cyle. We interpret the fraction of the M67 sample that is outside the cycle range as indicative of the frequency and nature of excursions in the solar cycle to either enhanced levels of activity or to exceptionally quiescent episodes reminiscent of a Maunder Minimum.

The HK index distribution in the M67 solar-type stars is not bimodal, in contrast to the earlier study by Baliunas \& Jastrow (1990). We suggest that our result is a manifestation of a single-mode of dynamo operation with occasional extensions in amplitude to either enhanced levels of activity or unusually quiescent episodes.

The inferred range in the possible amplitudes of long-term brightness changes extends from sun-like at $\sim 0.1 \%$ to as high as $3 \%$, or similar to the amplitude of photometric variability in Hyades-age stars (Radick et al. 1995).

Seasonal variability is observed in the program objects with a mean rms deviation in the HK index that is more than a factor of 2 higher than what is observed in the contemporary solar cycle. In general, the amplitude of variation in chromospheric activity is higher in the M67 solar-type stars than in the Sun. We have not yet identified conclusively the occurrence of cycle variability analogous to that of the solar cycle though some program objects exhibit long-term trends suggestive of cycle-like variability. In any event, if activity cycles are present then our data indicate that the cycle periods are greater than $\sim 6$ years. 
The chromospheric age range in the M67 sample extends from less than 1 Gyr to about 7.5 Gyr. This result is indicative of the magnitude of the impact of intrinsic chromospheric variations on age determinations based on the observed levels of activity in a stellar sample that is ostensibly homogeneous in age. We estimate a mean chromospheric age for M67 in the range of approximately $3.8-4.3$ Gyr.

Our intriguing results pose crucial questions. In particular, is the HK index distribution of chromospheric activity in the sun-like stars in M67 really the result of the long-term modulation of activity by cycles analogous to the solar cycle, or are the relative amplitudes of the cycles actually similar with the differences due only to differences in the mean level of activity, perhaps resulting from a dispersion in rotational velocities? Even more fundamentally, is the range and variability of chromospheric activity as seen in the cores of the $\mathrm{H}$ and $\mathrm{K}$ lines arising from sunlike cycles at all? These critical issues can only be addressed through regular observations of the "suns of M67" over a period of several years. Ideally, such a program should be conducted at echelle resolutions in order to more precisely measure the line strength and line profile variations in the cores between the $\mathrm{K}_{1}\left(\mathrm{H}_{1}\right)$ minima. In addition, high-precision differential photometry obtained in parallel with this kind of program would yield a direct determination of the nature of the joint variation of brightness and magnetic field-related activity.

The presence of very active solar-type stars in this solar-age cluster, that are also apparently single, is perplexing. We are in the process of attempting to obtain projected rotational velocity measurements in order to determine if it is rapid rotation that is the origin of the relatively enhanced activity in these objects. If this is verified to be the case then it would invite further investigation, both theoretical and observational, of the angular momentum history of M67 in contrast to that of the Sun and other solar-age G dwarfs. However, if the velocities are determined to be solar-like (i.e., $\sim 2 \mathrm{~km} \mathrm{~s}^{-1}$ ) then we can conclude that excursions to enhanced activity levels that are significantly in excess of contemporary solar maximum values occur in sun-like stars and perhaps in the Sun itself.

We are grateful to the NOAO Galactic TAC for its support of this long-term program. We are especially grateful to Dr. Diane Harmer for her assistance with the acquisition of the data for this investigation during the course of the WIYN Queue program and thereafter. We also acknowledge with appreciation the contributions by Mr. Daryl Willmarth, Dr. Paul Smith and Dr. Abi Saha who each obtained data for our investigation during the WIYN Queue program. We are particularly grateful to Dr. Bill Livingston for sharing with us his measurements of the solar $\mathrm{H}$ and $\mathrm{K}$ index from his multi-decadal program of integrated sunlight observations at the McMath-Pierce Solar telescope. We acknowledge interesting discussions with Dr. Claus Fröhlich and Dr. Peter Foukal during the course 
of this work. The authors thank Dr. Bob Mathieu for sharing in advance of publication his findings concerning binarity among solar-type stars in M67. We thank the referee, Dr. Doug Duncan, for his thoughtful review of the original manuscript. Finally, SLB gratefully acknowledges support by grants from the Richard C. Lounsbery Foundation and JPL grant P6201-7-05.

\section{REFERENCES}

Baliunas, S. L. \& Jastrow, R. 1990, Nature, 348, 520

Baliunas, S. L. et al. 1995, ApJ, 438, 269

Baliunas, S. L., Donahue, R. A., Soon, W \& Henry, G. W. 1998, ASP Conf. Series, vol. 154, p. 1534, p. 1534, p. 1534 , p. 153

Barden, S. C. \& Armandroff, T. 1995, SPIE, 2476, 56

Barry, D. C. \& Cromwell, R. H. 1974, ApJ, 187, 107

van den Berg, M., Tagliaferri, G., Belloni, T. \& Verbunt, F. 2004, A\&A, 418, 509

Belloni, T., Verbunt, F. \& Mathieu, R.D. 1998, A\&A, 339, 431

Cayrel de Stroebel, G. 1996, A\&A Rev., 7, 243

Cayrel de Stroebel, G. \& Bentolilla, C. 1989, A\&A, 211, 324

Damon, P. E. 1977, in The Solar Output and its Variation, ed. O. R. White (Boulder: Colorado Associated University Press), 429

Demarque, P., Guenther, D. B., \& Green, E. M. 1992, AJ, 103, 151

Donahue, R. A. 1993, PhD thesis, New Mexico State University, Las Cruces

Donahue, R. A. 1998, in the Proceedings of The 10th Cambridge Workshop on Cool Stars, Stellar Systems and the Sun, ASP Conf. Ser., vol. 154, eds. R. A. Donahue \& J. A. Bookbinder, (San Francisco: ASP), p. 1235

de Toma, G. et al. 2004, ApJ, 609, 1140

Eddy, J.A. 1976, Science, 192, 1189

Foukal, P. 2003, EOS, 84, 205

Foukal, P. \& Lean, J. 1990, Science, 247, 556

Fröhlich, C. 1994, in The Sun as a Variable Star: Solar and Stellar Irradiance Variations, Proceedings from IAU Colloquium 143, eds. J.M. Pap, C. Fröhlich, H.S. Hudson, and S. Solanki, (Cambridge: Cambridge University Press), p.28 
Girard, T. M., Grundy, W. M., López, C. E. \& van Altena, W. F. 1989, AJ, 98, 227

Haigh, J. D. 2003, Phil. Trans. Royal Soc., 361, 95

Haigh, J. D. 2005, in The Sun, Solar Analogs and the Climate, eds. I. Rüedi, M. Güdel \& W. Schmutz (Berlin Heidelberg: Spring Verlag), 1-104

Hall, J. C. \& Lockwood, G. W. 1995, ApJ, 438, 404

Hall, J. C. \& Lockwood, G. W. 2004, ApJ, 614, 942

Hoyt, D. V. \& Schatten, K. H. 1997, The Role of the Sun in Climate Change (New York: Oxford University Press), $279 \mathrm{pp}$

Hudson, H. S. 1988, ARA\&A, 26, 473

Lean, J., Rottman, G., Harder, J. \& Kopp, G. 2005, Sol. Phys., 230, 27

Linsky, J. L. et al. 1979, ApJS, 41, 47

Livingston, W. C. 1994, in The Solar Engine and its Influence on the Terrestrial Atmosphere and Climate, ed. E. Ribes, p. 145

Livingston, W. \& Wallace, L. 2003, Sol. Phys., 212, 227

Lockwood, G. W., Skiff, B. A. \& Radick, R. R. 1997, ApJ, 484, 789

Massey, Ph. 1997, A Quick Guide to Reducing WIYN Hydra Data, NOAO publication

Montgomery, K. A., Marschall, L. A., \& Janes, K. A. 1993, AJ, 106, 181

Noyes, R. W. et al. 1984, ApJ, 279, 763

Radick, R. R. 1991, in The Sun in Time, eds. C. P. Sonett, M. S. Giampapa \& M. S. Matthews (Tucson: University of Arizona Press), p. 787

Radick, R. R. 1995, ApJ, 452, 332

Ribes, J. C. \& Nesme-Ribes, E. 1993, A\&A, 276, 549

Rind, D. 2002, Science, 296. 673

Saar, S. H. 2002, in Stellar Coronae in the Chandra and XMM-Newton Era, ASP Conf. Ser., vol. 277, eds. F. Favata \& J. L. Drake (San Francisco: ASP), p. 311

Saar, S. H. \& Baliunas, S. L. 1992, in The Solar Cycle, ASP Conf. Ser., vol. 27, ed. K. L. Harvey (San Francisco: ASP), p. 197

Sanders, W. L. 1989, Rev. Mexicana Astron. Astrofis., 17, 31

Simon, T. 1992, in Proceedings of the 7th Cambridge Workshop on Cool Stars, Stellar Systems and the Sun, ASP Conf. Ser., vol. 26, eds. M. S. Giampapa \& J. A. Bookbinder (San Francisco: ASP), p. 3 
Skumanich, A. 1972, ApJ, 171, 565

Soderblom, D. R., Duncan, D. K. \& Johnson, D.R.H. 1991, ApJ, 375, 722

Soon, W. H., Posmentier, E. S. \& Baliunas, S. L. 1996, ApJ, 472, 891

Soon, W. H., Baliunas, S. L., Posmentier, E. S. \& Okeke, P. 2000a, New A, 4, 563

Soon, W. H., Posmentier, E. S. \& Baliunas, S. L. 2000b, Ann. Geophysicae, 18, 583

Soon, W. H. \& Yaskell, S. H. 2004, The Maunder Minimum and the Variable Sun-Earth Connection (Singapore: World Scientific), 269 pp

Stauffer, J. R. et al. 1991, ApJ, 374, 142

VandenBerg, D. A. \& Bridges, T. J. 1984, ApJ, 278, 679

Vaughan, A. H., Preston, G. W. \& Wilson, O. C. 1978, PASP, 90, 267

Walter, F.M. \& Barry, D.C. 1991, in the Sun in Time, eds. C. P. Sonett, M. S. Giampapa \& M. S. Matthews (Tucson: University of Arizona Press), p. 633

Wang, Y.-M., Lean, J. L. \& Sheeley, N. R., Jr. 2005, ApJ, 625, 522

White, O. 1978, in The Solar Output and its Variation, ed. O. R. White (Boulder: Colorado Associated University Press), p. 44

White, O. R. \& Livingston, W. C. 1978, ApJ, 226, 679

White, O. R. \& Livingston, W. C. 1981, ApJ, 249, 798

Wilson, O.C. 1978, ApJ, 226, 379

Wright, J. T. 2004, AJ, 128, 1273

Zhang, Q., Soon, W. H., Baliunas, S. L. \& Lockwood, G. W. 1994, ApJ, 427, L111 
Table 1. Survey Results

\begin{tabular}{|c|c|c|c|c|c|c|c|}
\hline $\begin{array}{c}\text { Sanders } \\
\text { No. }\end{array}$ & $(B-V)_{o}$ & $\begin{array}{c}\mathrm{HK} \\
(\mathrm{m} \AA)\end{array}$ & $\begin{array}{l}\text { Error } \\
(\mathrm{m} \AA)\end{array}$ & V & $\mathrm{P}_{\mu}$ & $\mathrm{P}_{\mu, r}$ & binarity $^{\mathrm{a}}$ \\
\hline 603 & 0.56 & 208 & 9.99 & 14.05 & 97 & 96 & - \\
\hline 621 & 0.60 & 200 & 8.3 & 14.44 & 87 & 86 & - \\
\hline 724 & 0.63 & 200 & 10.8 & 14.54 & 93 & 85 & - \\
\hline 746 & 0.66 & 209 & 8.58 & 14.38 & 94 & 96 & - \\
\hline 747 & 0.65 & 354 & 10.9 & 14.052 & 90 & 95 & - \\
\hline 748 & 0.78 & 264 & 12.3 & 14.632 & 89 & 94 & $\mathrm{y}$ \\
\hline 753 & 0.58 & 208 & 7.49 & 14.693 & 93 & 96 & $* *$ \\
\hline 770 & 0.63 & 195 & 7.43 & 14.636 & 88 & 96 & $* *$ \\
\hline 777 & 0.63 & 208 & 8.7 & 14.52 & 93 & 96 & $* *$ \\
\hline 779 & 0.68 & 190 & 8.34 & 14.43 & 85 & 94 & $* *$ \\
\hline 785 & 0.65 & 221 & 8.24 & 14.823 & 88 & 95 & $* *$ \\
\hline 789 & 0.61 & 204 & 5.99 & 14.054 & 85 & 95 & - \\
\hline 801 & 0.67 & 182 & 6.71 & 15.094 & 87 & 95 & $* *$ \\
\hline 802 & 0.67 & 193 & 8.08 & 14.792 & 86 & 93 & $* *$ \\
\hline 829 & 0.54 & 172 & 7.49 & 14.37 & 91 & 87 & - \\
\hline 937 & 0.59 & 194 & 8.52 & 14.12 & 95 & 88 & - \\
\hline 942 & 0.58 & 206 & 11.5 & 14.475 & 90 & 87 & - \\
\hline 943 & 0.71 & 166 & 18 & 14.96 & 85 & 82 & - \\
\hline 945 & 0.62 & 205 & 7.65 & 14.528 & 93 & 92 & $* *$ \\
\hline 951 & 0.67 & 190 & 7.09 & 14.704 & 87 & 87 & $\mathrm{y}$ \\
\hline 958 & 0.62 & 206 & 8.52 & 14.45 & 96 & 97 & - \\
\hline 963 & 0.66 & 168 & 11.3 & 14.513 & 91 & 96 & $\mathrm{P}=90.28$ \\
\hline 965 & 0.71 & 222 & 11.6 & 14.695 & 80 & 90 & $* *$ \\
\hline 969 & 0.62 & 203 & 9.07 & 14.177 & 87 & 95 & $* *$ \\
\hline 981 & 0.66 & 177 & 14.9 & 14.16 & 96 & 99 & $\mathrm{P}=56.0$ \\
\hline 982 & 0.56 & 231 & 6.99 & 14.122 & 94 & 99 & $\mathrm{P}=373$ \\
\hline 991 & 0.63 & 179 & 8.67 & 14.564 & 91 & 97 & $* *$ \\
\hline 1004 & 0.71 & 214 & 10.5 & 14.928 & 89 & 98 & 2 \\
\hline 1012 & 0.69 & 228 & 10.3 & 14.516 & 88 & 98 & $\mathrm{P}=641$ \\
\hline 1014 & 0.66 & 248 & 13.8 & 14.183 & 86 & 98 & $\mathrm{P}=16.2$ \\
\hline
\end{tabular}


Table 1-Continued

\begin{tabular}{|c|c|c|c|c|c|c|c|}
\hline $\begin{array}{c}\text { Sanders } \\
\text { No. }\end{array}$ & $(B-V)_{o}$ & $\begin{array}{c}\mathrm{HK} \\
(\mathrm{m} \AA)\end{array}$ & $\begin{array}{l}\text { Error } \\
(\mathrm{m} \AA)\end{array}$ & V & $\mathrm{P}_{\mu}$ & $\mathrm{P}_{\mu, r}$ & binarity $^{a}$ \\
\hline 1033 & 0.56 & 209 & 8.69 & 14.164 & 97 & 99 & $* *$ \\
\hline 1041 & 0.68 & 193 & 9.51 & 14.718 & 83 & 97 & 2 \\
\hline 1048 & 0.64 & 188 & 8.95 & 14.411 & 92 & 99 & - \\
\hline $1050^{\mathrm{d}}$ & 0.61 & 390 & 19.7 & 14.292 & 84 & 97 & $\mathrm{y}$ \\
\hline 1057 & 0.63 & 210 & 7.03 & 14.303 & 81 & 96 & $* *$ \\
\hline 1064 & 0.61 & 178 & 11.2 & 14.04 & 94 & 99 & $\mathrm{P}=575$ \\
\hline 1065 & 0.75 & 222 & 8.99 & 14.645 & 77 & 93 & $\mathrm{P}=150$ \\
\hline 1068 & 0.70 & 211 & 7.84 & 15.028 & 83 & 95 & 2 \\
\hline 1078 & 0.61 & 190 & 7.13 & 14.174 & 97 & 99 & $* *$ \\
\hline 1087 & 0.59 & 198 & 8.13 & 14.16 & 84 & 94 & - \\
\hline 1089 & 0.62 & 173 & 7.31 & 14.199 & 91 & 96 & - \\
\hline 1093 & 0.59 & 207 & 8.94 & 14.134 & 83 & 91 & - \\
\hline 1095 & 0.61 & 183 & 6.74 & 14.59 & 88 & 90 & $* *$ \\
\hline 1096 & 0.62 & 192 & 7.23 & 14.49 & 94 & 95 & - \\
\hline 1106 & 0.67 & 168 & 9.72 & 14.78 & 91 & 90 & - \\
\hline 1107 & 0.55 & 241 & 7.5 & 14.13 & 96 & 95 & - \\
\hline $1112^{\mathrm{b}}$ & 0.69 & 729 & 18.5 & 15.05 & 92 & 86 & - \\
\hline $1203^{\mathrm{c}}$ & 0.67 & 218 & 10.4 & 14.413 & 97 & 97 & - \\
\hline 1208 & 0.79 & 229 & 18.9 & 14.6 & 92 & 95 & $\mathrm{P}=19.9$ \\
\hline 1212 & 0.73 & 218 & 13.1 & 15.32 & 83 & 86 & 1 \\
\hline 1213 & 0.55 & 189 & 8.64 & 14.114 & 98 & 99 & - \\
\hline 1218 & 0.63 & 194 & 9.7 & 14.59 & 97 & 99 & $* *$ \\
\hline 1246 & 0.64 & 187 & 9.65 & 14.623 & 82 & 95 & $* *$ \\
\hline 1247 & 0.57 & 222 & 5.59 & 14.044 & 95 & 99 & $\mathrm{P}=69.8$ \\
\hline 1248 & 0.57 & 202 & 8.82 & 14.22 & 96 & 99 & $* *$ \\
\hline 1249 & 0.73 & 203 & 9.31 & 14.314 & 92 & 97 & - \\
\hline 1251 & 0.70 & 231 & 10.4 & 14.79 & 81 & 90 & $* *$ \\
\hline 1252 & 0.59 & 201 & 8.14 & 14.067 & 97 & 99 & ** \\
\hline 1255 & 0.62 & 211 & 6.75 & 14.486 & 94 & 99 & $* *$ \\
\hline 1258 & 0.61 & 183 & 8.38 & 14.54 & 92 & 97 & $* *$ \\
\hline
\end{tabular}


Table 1 - Continued

\begin{tabular}{ccccccll}
\hline \hline $\begin{array}{c}\text { Sanders } \\
\text { No. }\end{array}$ & $(B-V)_{o}$ & $\begin{array}{c}\mathrm{HK} \\
(\mathrm{m} \AA)\end{array}$ & $\begin{array}{c}\text { Error } \\
(\mathrm{m} \AA)\end{array}$ & $\mathrm{V}$ & $\mathrm{P}_{\mu}$ & $\mathrm{P}_{\mu, r}$ & binarity $^{\mathrm{a}}$ \\
\hline 1260 & 0.58 & 202 & 8.2 & 14.191 & 97 & 99 & $* *$ \\
1269 & 0.71 & 183 & 8.18 & 14.934 & 81 & 93 & $* *$ \\
1278 & 0.73 & 181 & 11.9 & 14.401 & 91 & 98 & SB2, long \\
1289 & 0.71 & 176 & 6.61 & 14.901 & 94 & 99 & $* *$ \\
1307 & 0.76 & 172 & 13.2 & 15.167 & 80 & 91 & 1 \\
1318 & 0.51 & 217 & 7.05 & 14 & 90 & 92 & - \\
1330 & 0.55 & 238 & 8.57 & 14.04 & 82 & 77 & - \\
1341 & 0.62 & 233 & 8.65 & 14.68 & 94 & 80 & - \\
1406 & 0.55 & 201 & 7.75 & 14.02 & 96 & 87 & - \\
1420 & 0.56 & 205 & 8.29 & 14.15 & 95 & 92 & - \\
1426 & 0.57 & 197 & 8.6 & 14.25 & 91 & 82 & - \\
1446 & 0.57 & 207 & 7.42 & 14.036 & 74 & 72 & - \\
1449 & 0.61 & 198 & 11.7 & 14.381 & 96 & 97 & - \\
1452 & 0.62 & 414 & 11.1 & 14.578 & 86 & 88 & $* *$ \\
1462 & 0.63 & 193 & 6.98 & 14.37 & 97 & 98 & - \\
1473 & 0.73 & 158 & 11.5 & 15.055 & 83 & 86 & 2 \\
1477 & 0.67 & 181 & 9.12 & 14.593 & 91 & 93 & $* *$ \\
\hline
\end{tabular}

${ }^{a}$ R. D. Mathieu, private communication. A dash indicates that no radial velocity information is available; quoted periods $\mathrm{P}$ are in units of days; "SB2, long" denotes a long-period, double-line spectroscopic binary; 1 = one observation at the cluster mean radial velocity; 2 $=\mathrm{rms}<2 \mathrm{~km} \mathrm{~s}^{-1}$, with observations in two years; $\mathrm{y}=$ binary, no orbital elements; ${ }^{* *}=$ $\mathrm{rms}<2 \mathrm{~km} \mathrm{~s}^{-1}$

${ }^{\mathrm{b}}$ ROSAT X-ray source and possible RS CVn; Belloni et al. (1998). Object not included in analysis presented herein.

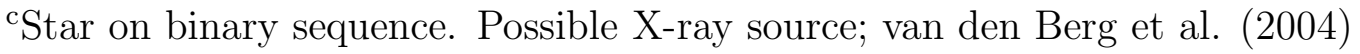

${ }^{\mathrm{d} C h a n d r a}$ X-ray source; van den Berg et al. (2004) 
Table 2. Chromospheric Variability of M67 Solar-Type Stars

\begin{tabular}{ccc}
\hline \hline $\begin{array}{c}\text { Sanders } \\
\text { No. }\end{array}$ & $(B-V)_{o}$ & $\begin{array}{c}\text { RMS a } \\
(\mathrm{mA})\end{array}$ \\
\hline 621 & 0.60 & 12.7 \\
724 & 0.63 & 22.4 \\
746 & 0.66 & 9.55 \\
747 & 0.65 & 22.0 \\
770 & 0.63 & 13.0 \\
777 & 0.63 & 16.0 \\
779 & 0.68 & 8.44 \\
785 & 0.65 & 14.0 \\
789 & 0.61 & 4.6 \\
801 & 0.67 & 15.0 \\
802 & 0.67 & 7.66 \\
937 & 0.59 & 8.16 \\
942 & 0.58 & 16.4 \\
945 & 0.62 & 21.6 \\
958 & 0.62 & 14.3 \\
963 & 0.66 & 15.0 \\
965 & 0.71 & 13.3 \\
969 & 0.62 & 19.4 \\
981 & 0.66 & 5.00 \\
991 & 0.63 & 14.7 \\
1004 & 0.71 & 30.3 \\
1014 & 0.66 & 40.0 \\
1041 & 0.68 & 31.3 \\
1048 & 0.64 & 16.3 \\
1057 & 0.62 & 11.7 \\
1064 & 0.61 & 23.0 \\
1068 & 0.70 & 57.0 \\
1078 & 0.61 & 7.62 \\
1087 & 0.59 & 14.6 \\
1089 & 0.62 & 26.9 \\
& & \\
\hline
\end{tabular}


Table 2-Continued

\begin{tabular}{lcc}
\hline \hline \multicolumn{1}{c}{ Sanders } & $(B-V)_{o}$ & $\begin{array}{c}\text { RMS } \\
\text { No. }\end{array}$ \\
\hline 1093 & & $(\mathrm{~m})$ \\
1095 & 0.59 & 15.8 \\
1096 & 0.61 & 16.6 \\
1106 & 0.62 & 8.66 \\
1112 & 0.67 & 22.6 \\
1203 & 0.69 & 92.1 \\
1212 & 0.67 & 36.3 \\
1218 & 0.73 & 32.0 \\
1246 & 0.63 & 18.5 \\
1249 & 0.64 & 20.3 \\
1251 & 0.73 & 10.0 \\
1252 & 0.70 & 37.5 \\
1255 & 0.59 & 17.7 \\
1258 & 0.62 & 6.16 \\
1260 & 0.61 & 30.8 \\
1269 & 0.58 & 13.4 \\
1278 & 0.71 & 17.2 \\
1289 & 0.73 & 12.0 \\
1307 & 0.71 & 8.50 \\
1341 & 0.76 & 23.4 \\
1449 & 0.62 & 11.7 \\
1452 & 0.61 & 14.1 \\
1462 & 0.62 & 26.4 \\
1477 & 0.63 & 33.8 \\
Sun $(1976-04)$ & 0.65 & 7.90 \\
Sun $(1976-82)$ & 0.65 & 9.02 \\
Sun $(1982-88)$ & 0.65 & 6.07 \\
& 0.65 & 7.49 \\
$1988-94)$ & 0.65 & 5.93 \\
\hline
\end{tabular}


Table 2-Continued

\begin{tabular}{ccc}
\hline \hline $\begin{array}{c}\text { Sanders } \\
\text { No. }\end{array}$ & $(B-V)_{o}$ & $\begin{array}{c}\text { RMS a } \\
(\mathrm{m} \AA)\end{array}$ \\
\hline Sun (1998-04) & 0.65 & 4.64 \\
\hline
\end{tabular}

${ }^{a}$ Root mean square deviation of the seasonal values of the HK index 
Fig. 1. - Color-magnitude diagram of M67 with the program objects indicated by filled circles. Stars in our survey that are known binaries are denoted by $\otimes$. Open circles are proper motion members with membership probabilities $\geq 90 \%$

Fig. 2.- A comparison of the solar spectrum in the $\mathrm{Ca}$ II $\mathrm{H}$ and $\mathrm{K}$ line region with the spectrum of an M67 solar-type star (solid line) that has been calibrated according to the procedure described in $\S 3$. The solar spectrum (dash line), which is from the NSO Solar Atlas, has been degraded to the resolution of the stellar spectrum

Fig. 3. - The distribution of the HK index for the M67 solar-type stars (solid). Also shown is the HK index distribution for the contemporary solar cycle, based on measurements by W. C. Livingston from 1976 to 2004 using the NSO McMath-Pierce Solar Telescope on Kitt Peak (dash). The solar data have been adjusted in this comparison to take into account the effect of the differences in spectral resolution between the solar and stellar data (see $\S 3$ for a discussion). The solar histogram has been normalized for plotting purposes

Fig. 4. - The HK index as a function of intrinsic $B-V$ color for the solar-type stars in M67. Note the increase in scatter toward cooler stars

Fig. 5.- A sequence of histograms showing the distribution of HK index in bins of $B-V$ color for the 60 solar-type stars observed in M67. The solar cycle distribution (dash) is provided for comparative purposes. See $\S 4$ for a discussion

Fig. 6. - The HK index distribution of known binaries (solid) and stars that are not known to be binary systems (dash) in our M67 sample of solar-type stars. See $\S 4$ for a discussion

Fig. 7.- The HK index versus orbital period for the binaries in our sample. The periods were kindly provided in advance of publication by R. D. Mathieu

Fig. 8. - The cumulative probability functions in HK index for the solar-type stars in M67 (solid) and the modern solar cycle (dash), respectively. See $\S 5$ for a discussion

Fig. 9.- The seasonal variation of chromospheric activity in solar-type stars in M67. The Sun seen as a star is included for comparison. See $§ 5.2$ for a discussion

Fig. 10.- The appearance of the solar cycle variation in annual mean HK index. The solar HK index values are based on measurements provided by W. C. Livingston in advance of publication, and have been adjusted for the purposes of comparison with the M67 stellar data as discussed in $\S 3$. The dotted vertical grid lines are drawn at 6-year intervals to facilitate comparison of segments of the solar cycle of length similar to that of the record of seasonal variations of the M67 stars (Fig. 9) 
Fig. 11. - The root mean square deviation of the seasonal values of the HK index vs. mean HK index for the M67 solar-type stars in Table 2. See $\S 5.7$ for a discussion

Fig. 12. - The distribution of the seasonal values of the HK index (solid) along with the distribution of values seen in the modern solar cycle

Fig. 13. - The apparent age distribution as inferred from the current calibration of chromospheric activity and age for the M67 solar-type stars (solid). The corresponding distribution for the solar cycle is shown for comparison (dash). See $\S 5.3$ for a discussion

Fig. 14. - An example of an especially active solar-type star in M67. The emission reversals in the $\mathrm{Ca}$ II $\mathrm{H}$ and $\mathrm{K}$ cores are clearly evident in this object 


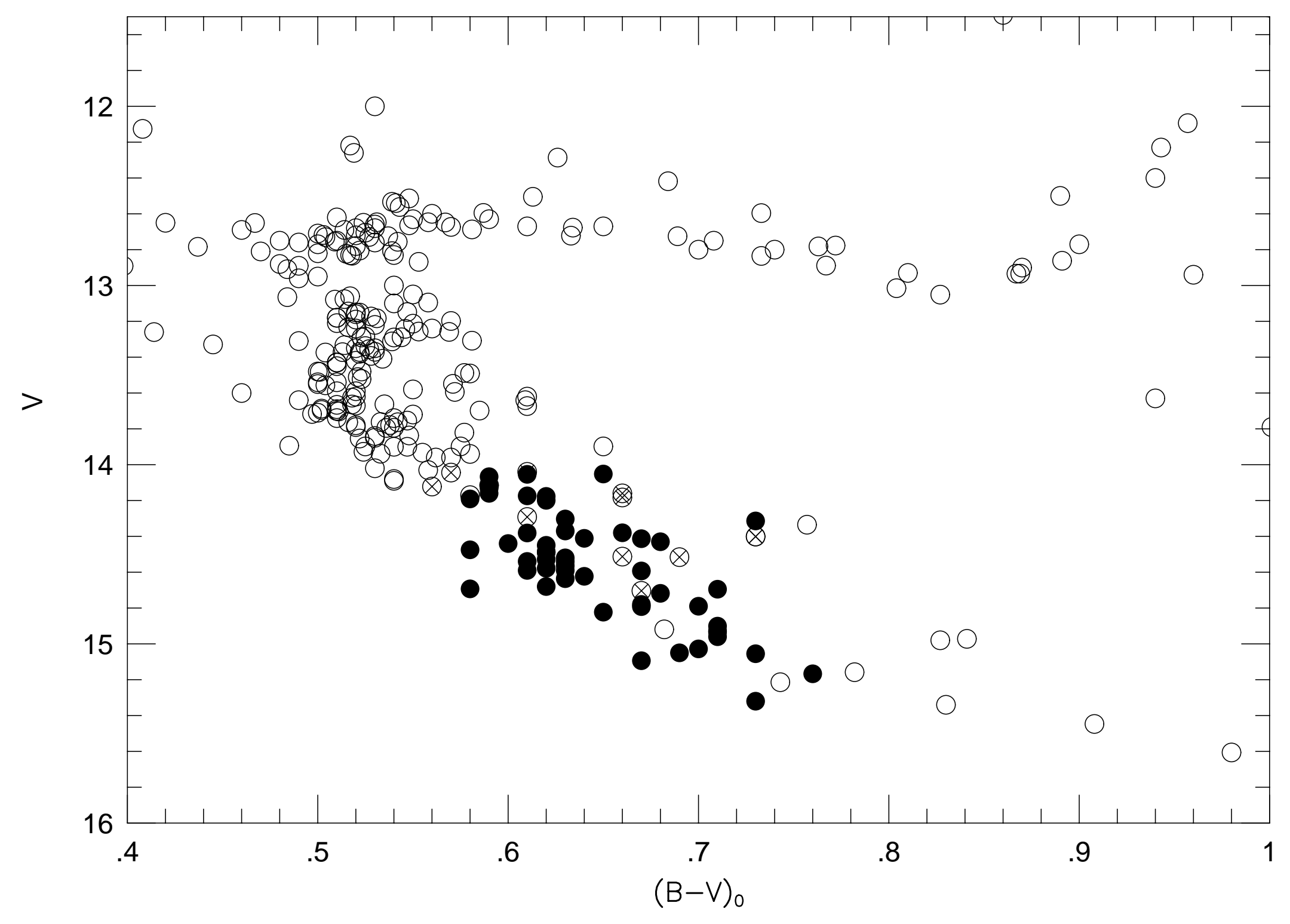




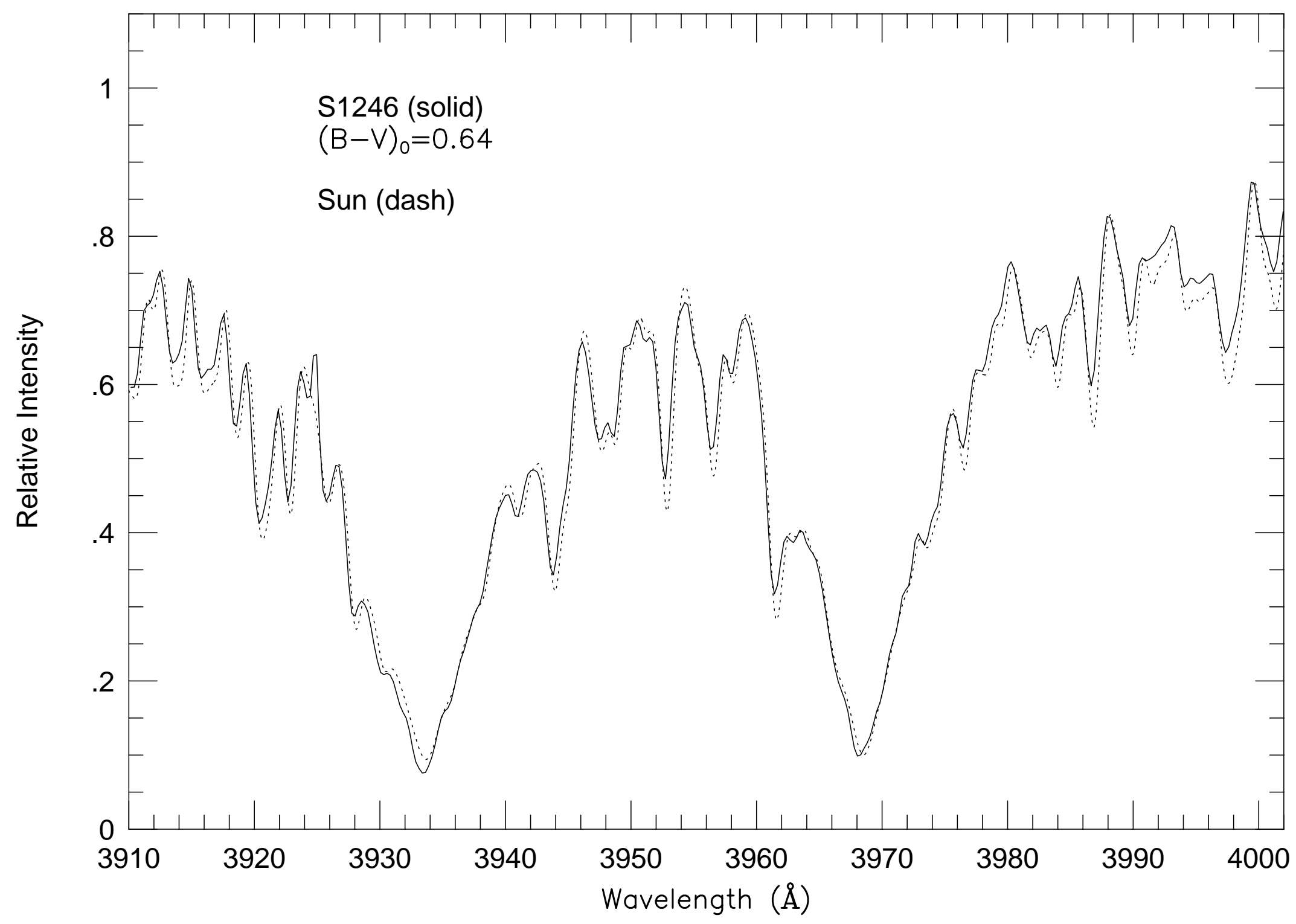




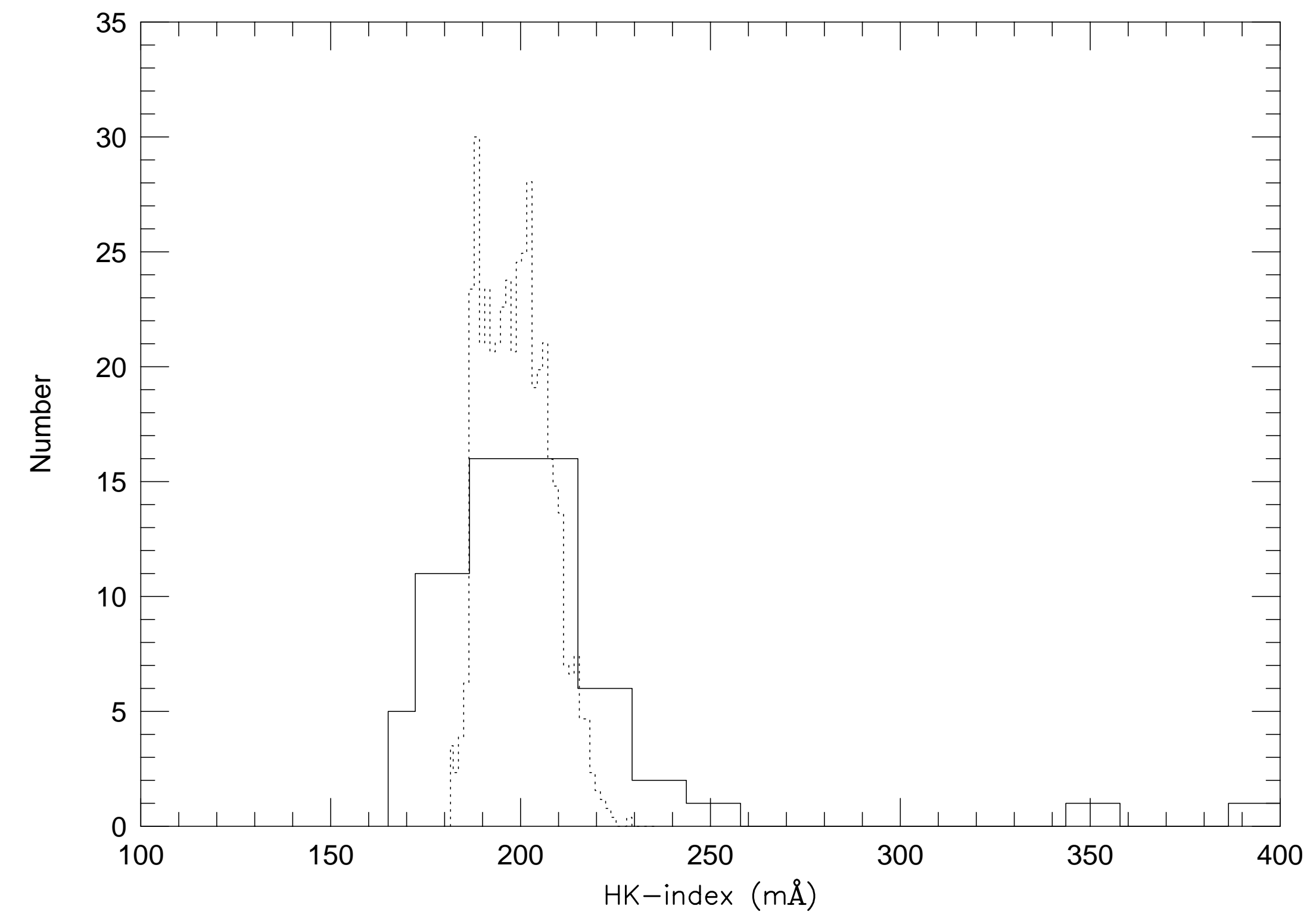




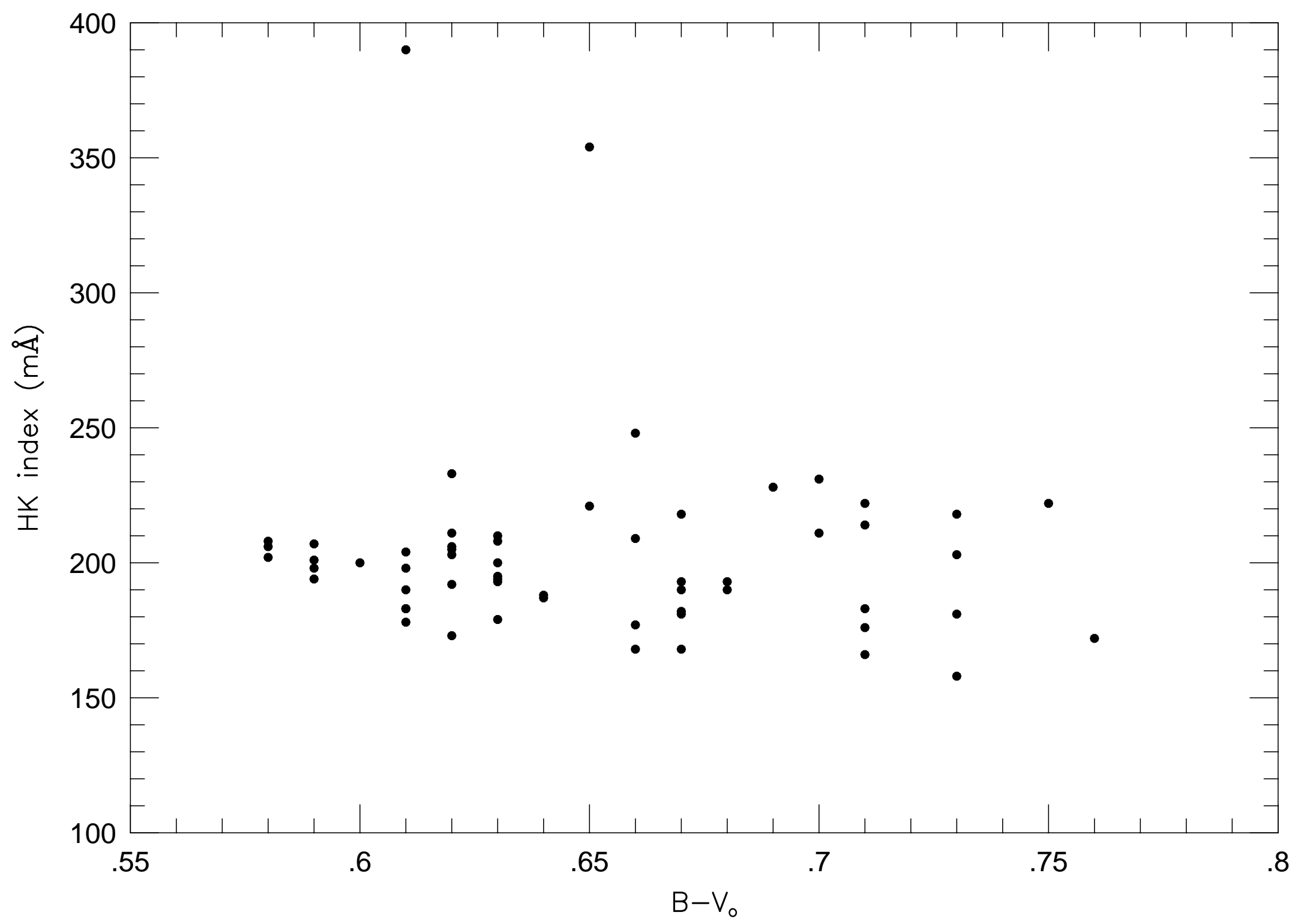



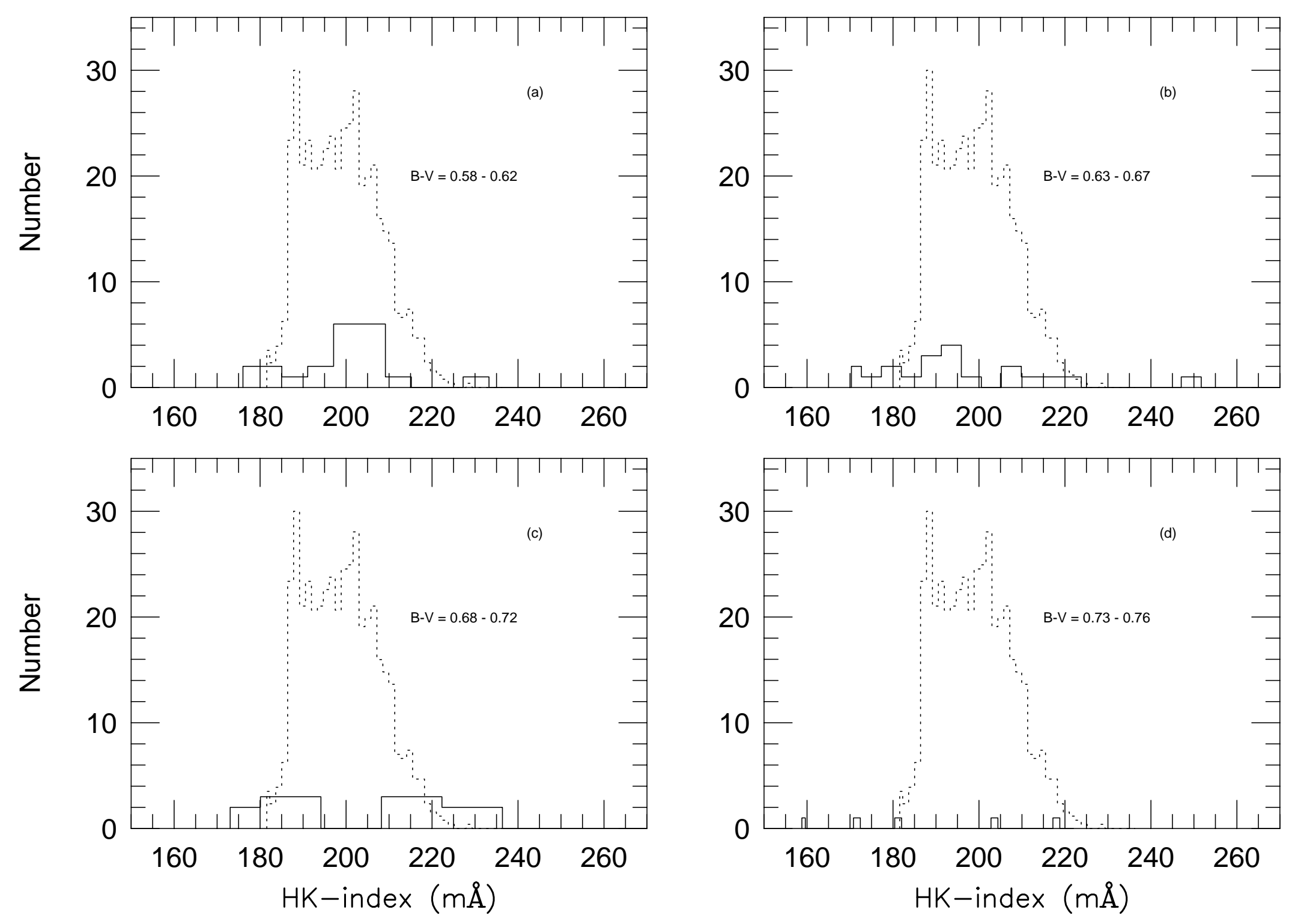


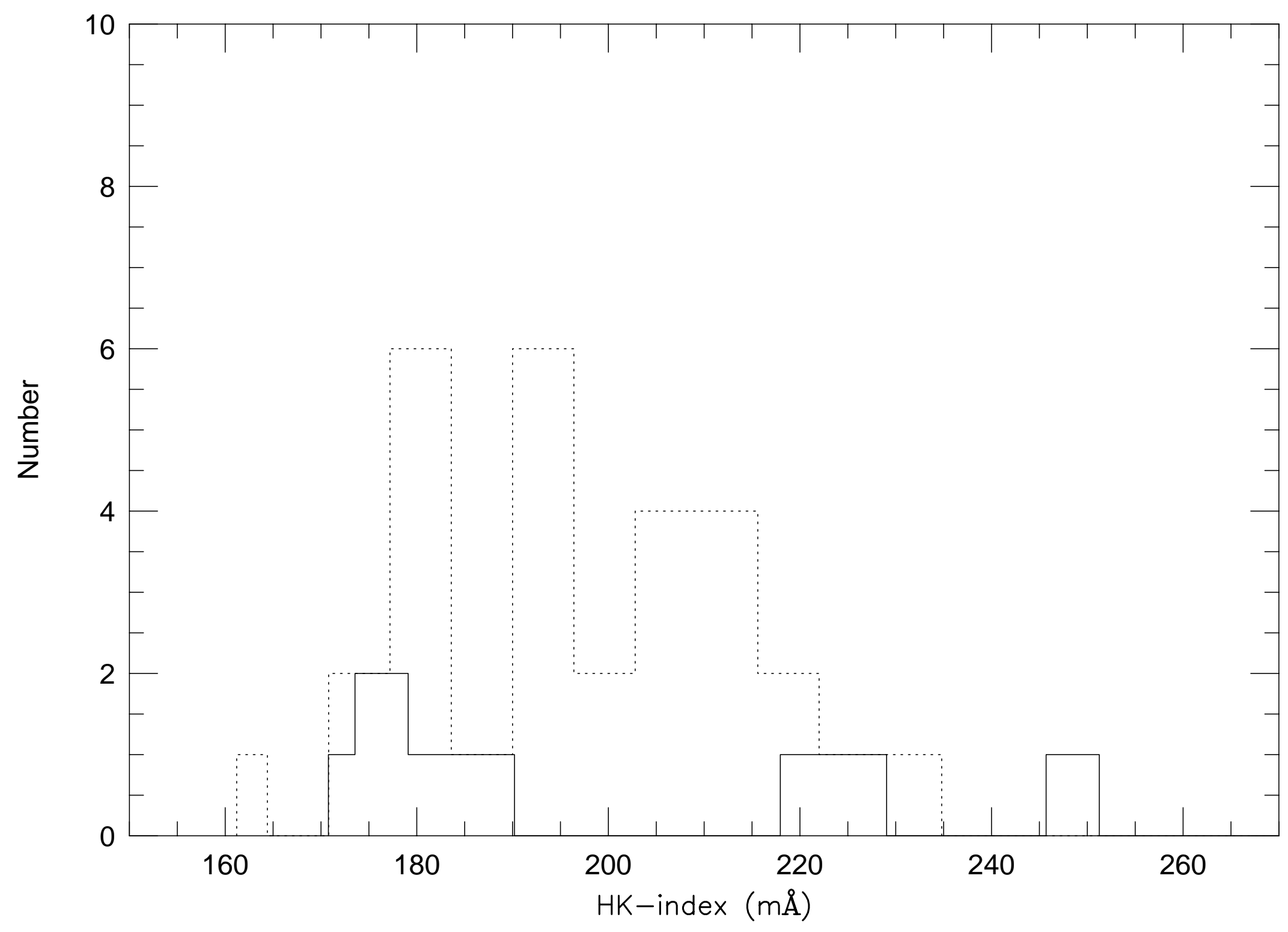




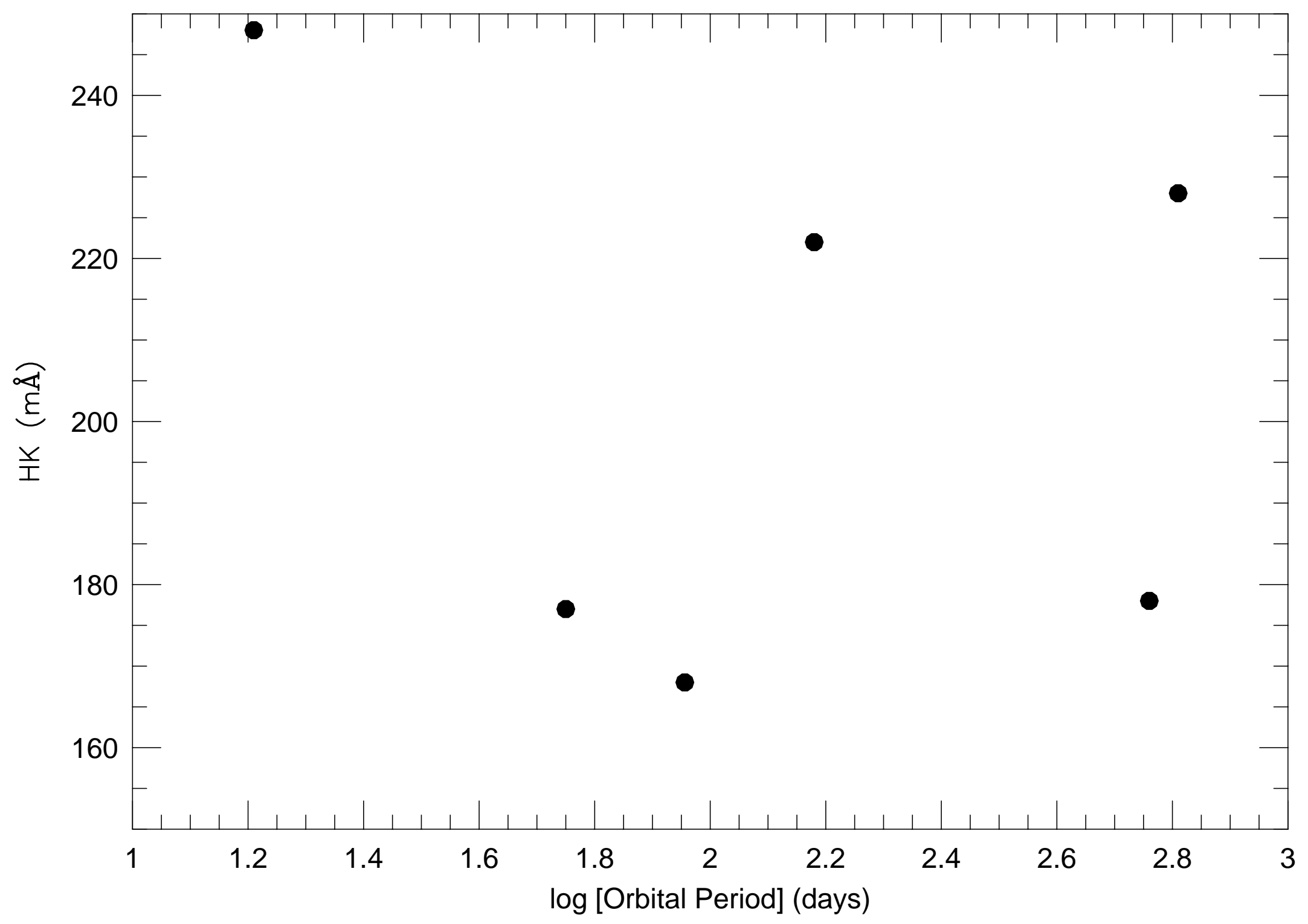




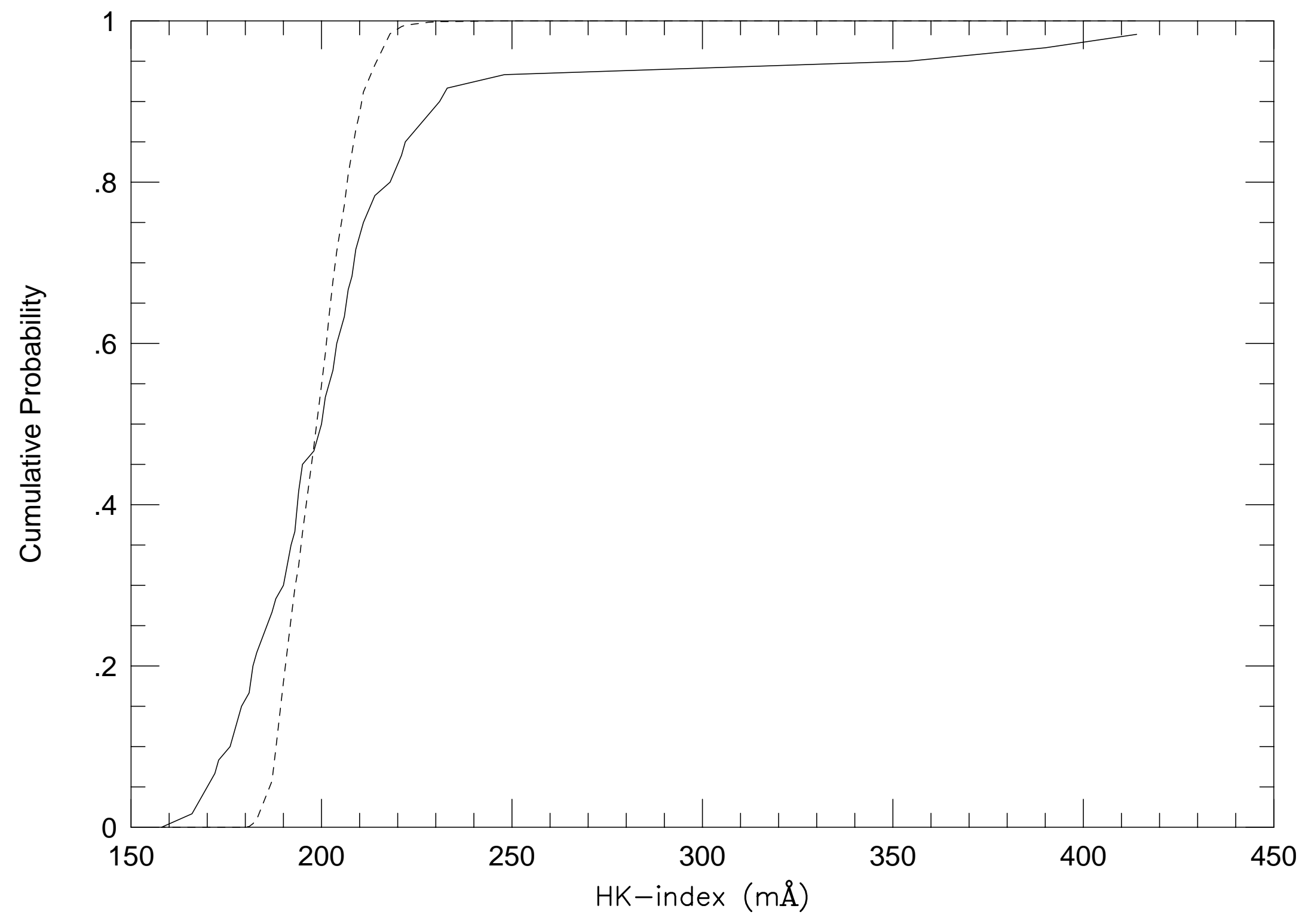


S942 (0.58)
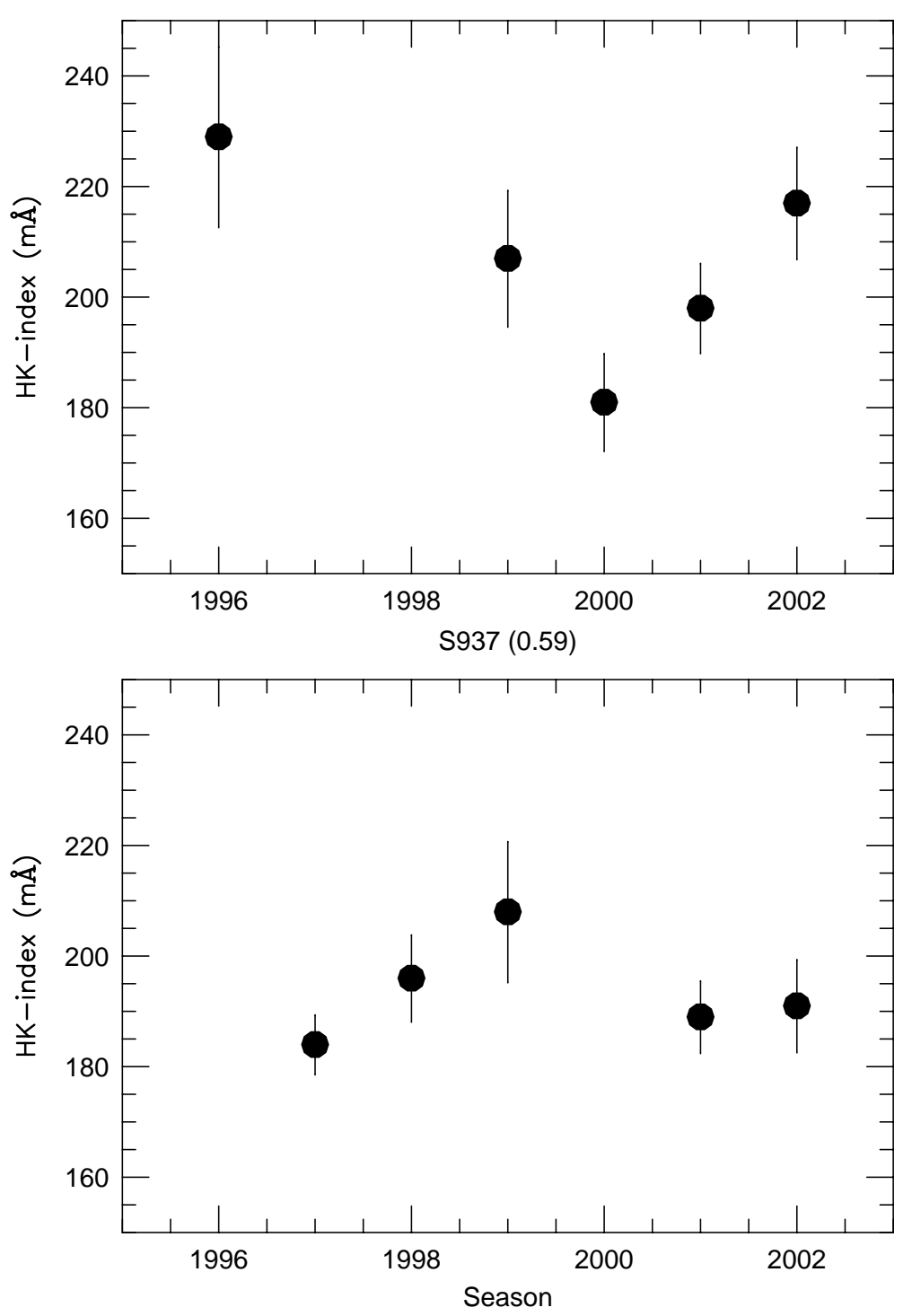

S1260 (0.58)
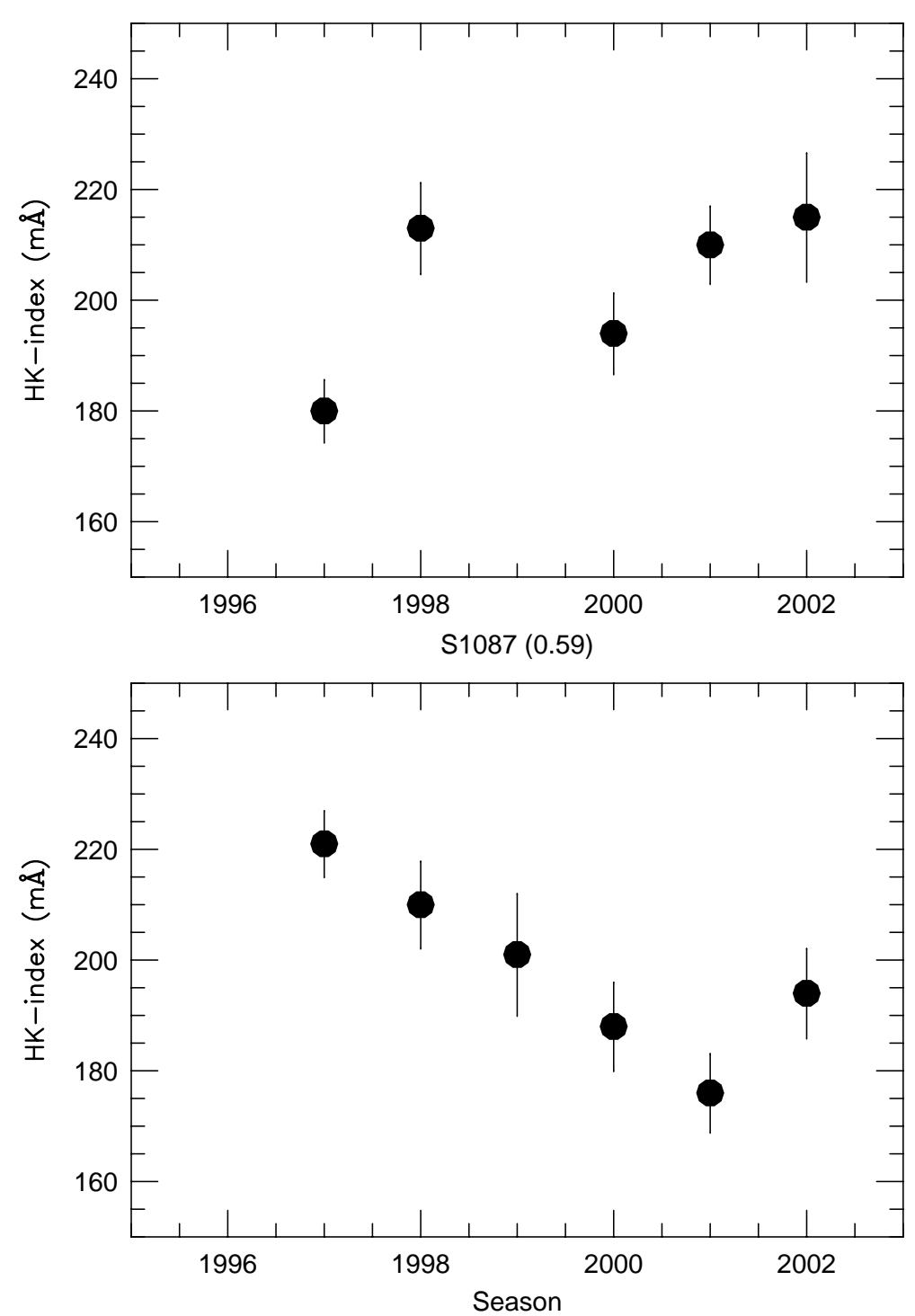
S1093 (0.59)
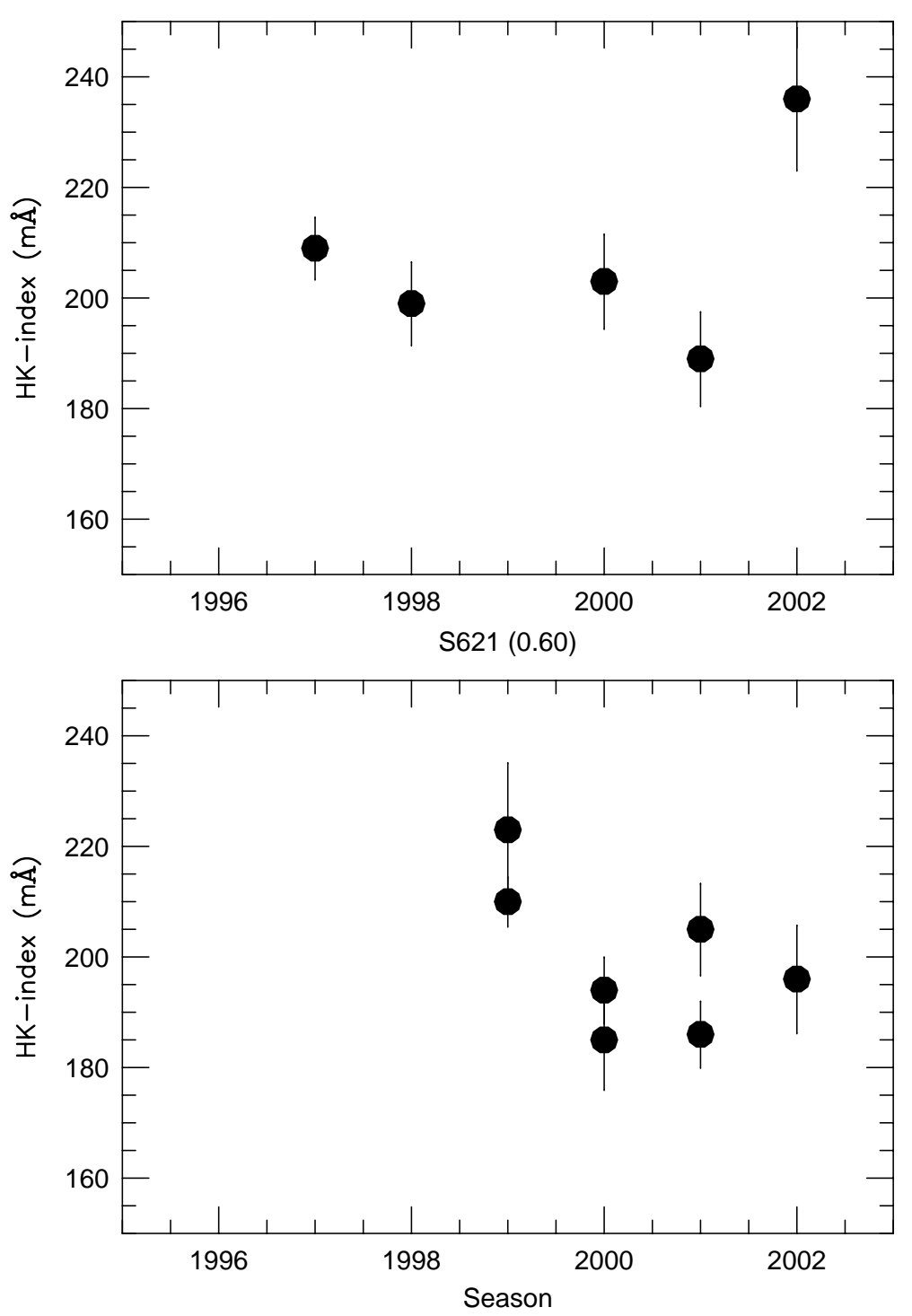

S1252 (0.59)
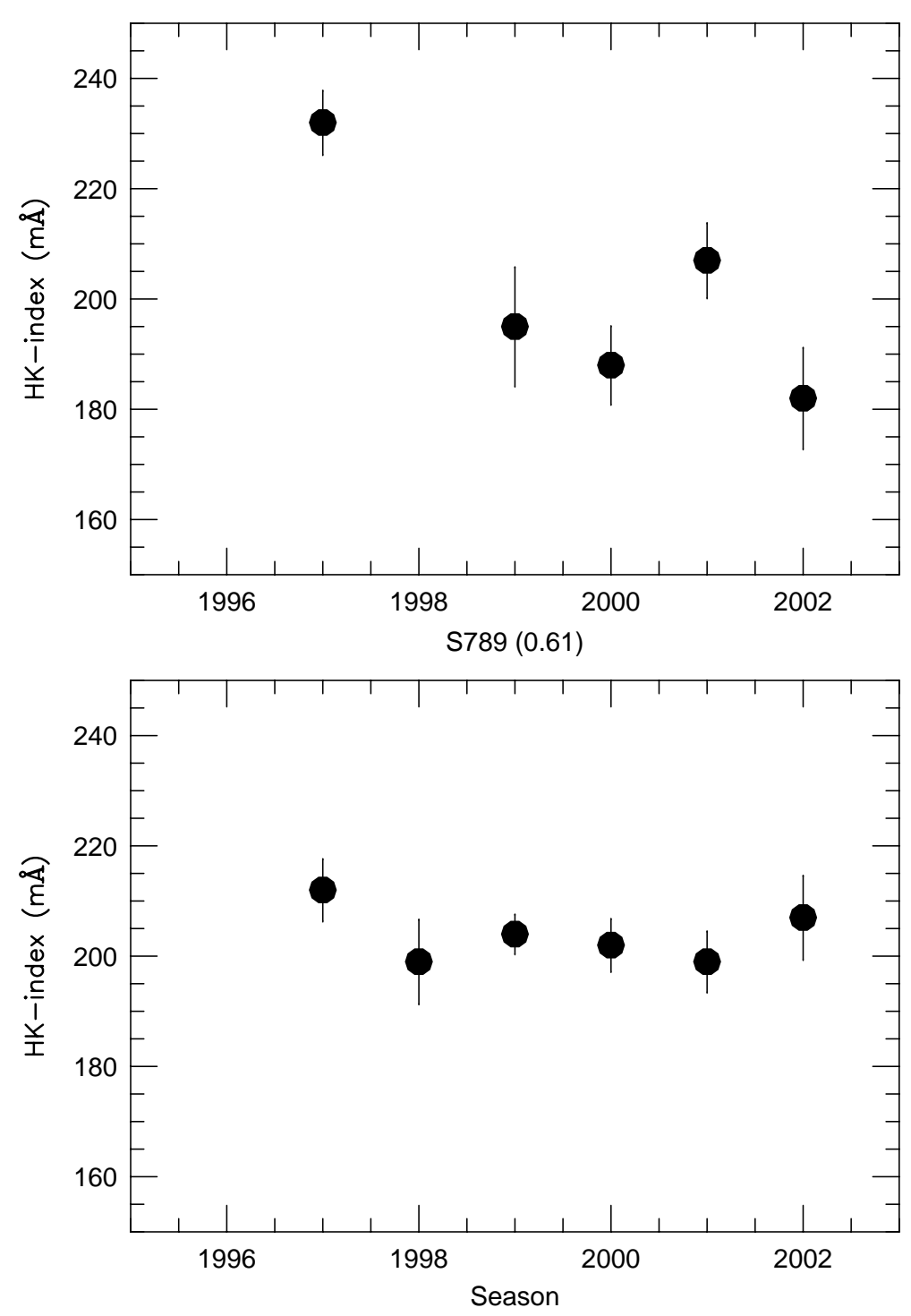
S1064 (0.61)

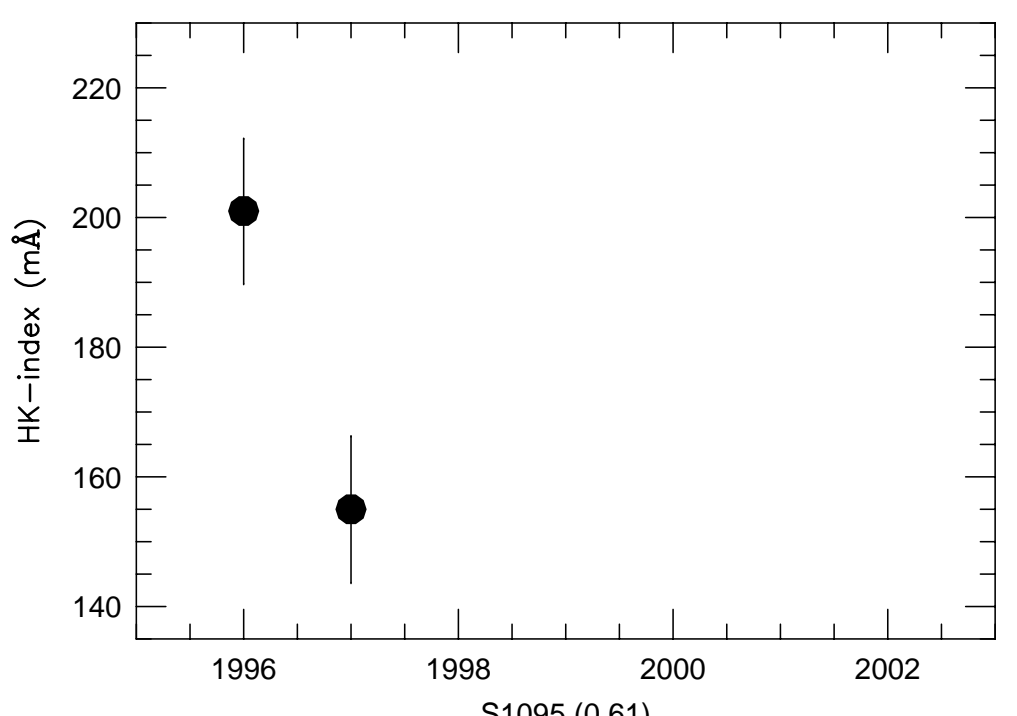

S1095 (0.61)

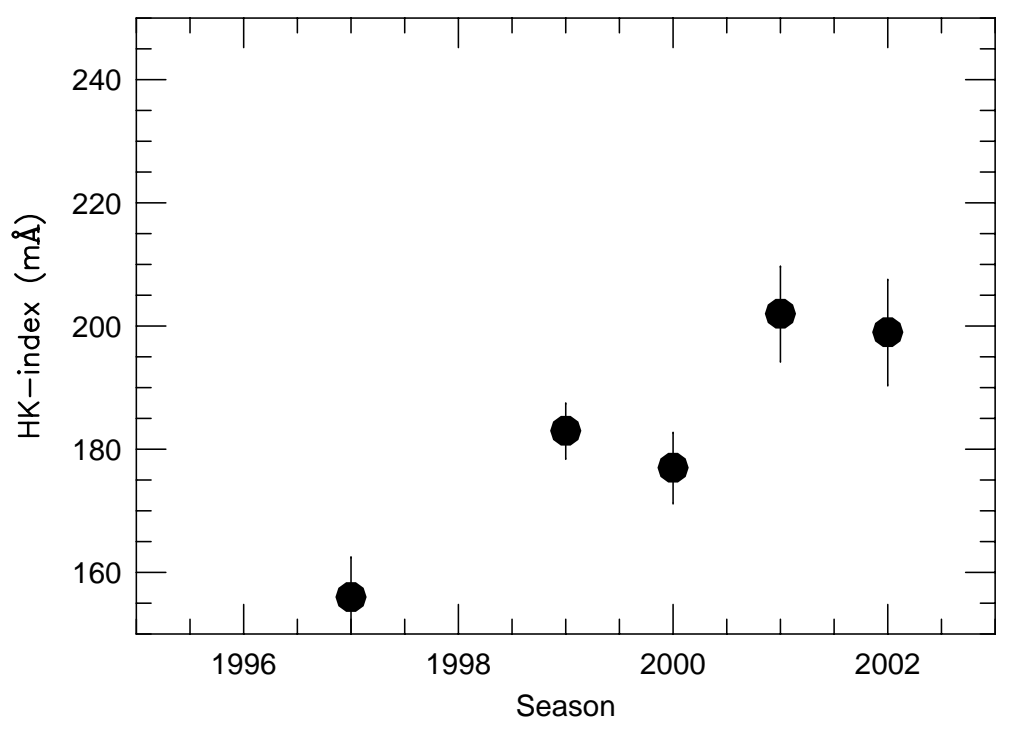

S1078 (0.61)
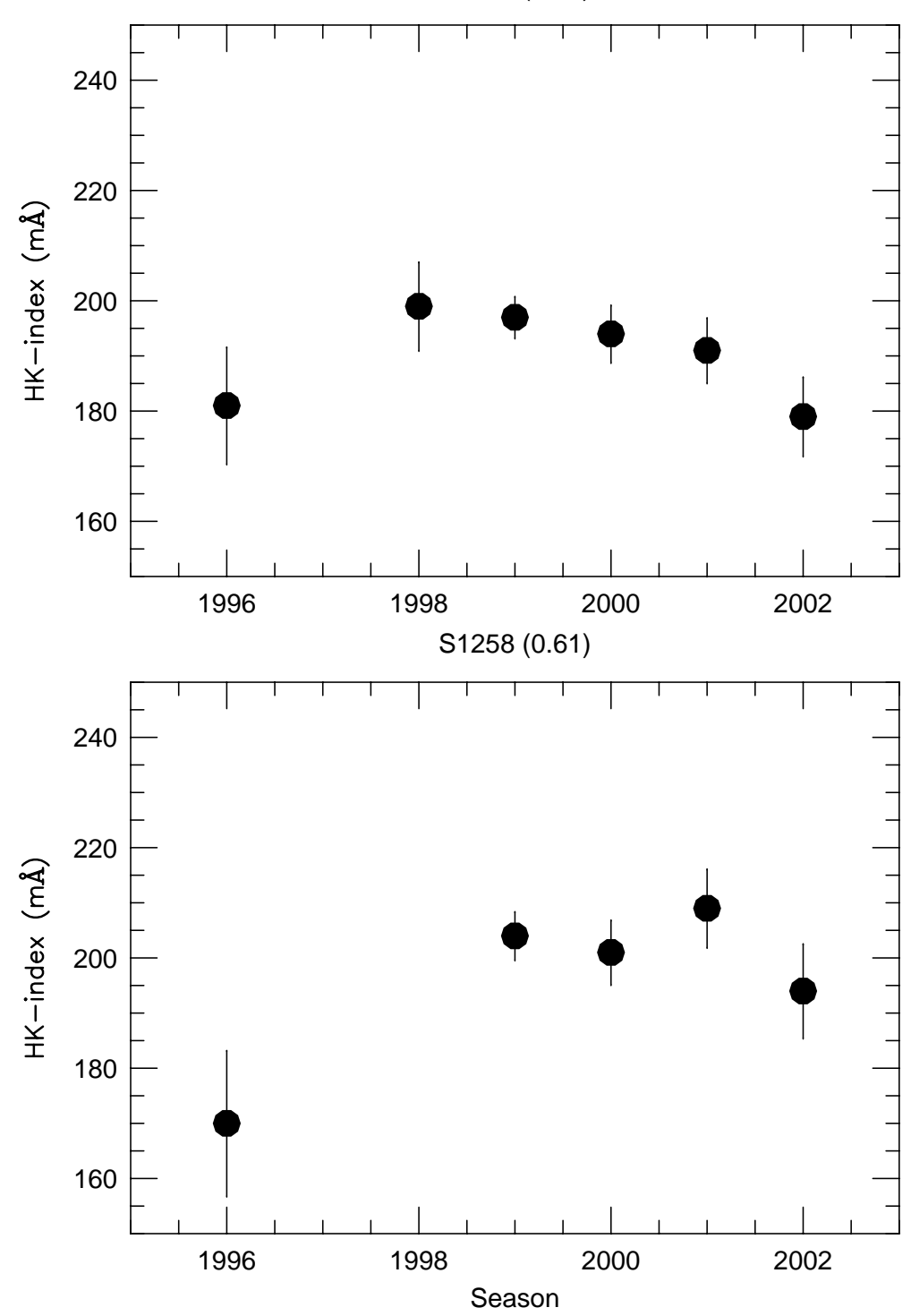
S1449 (0.61)

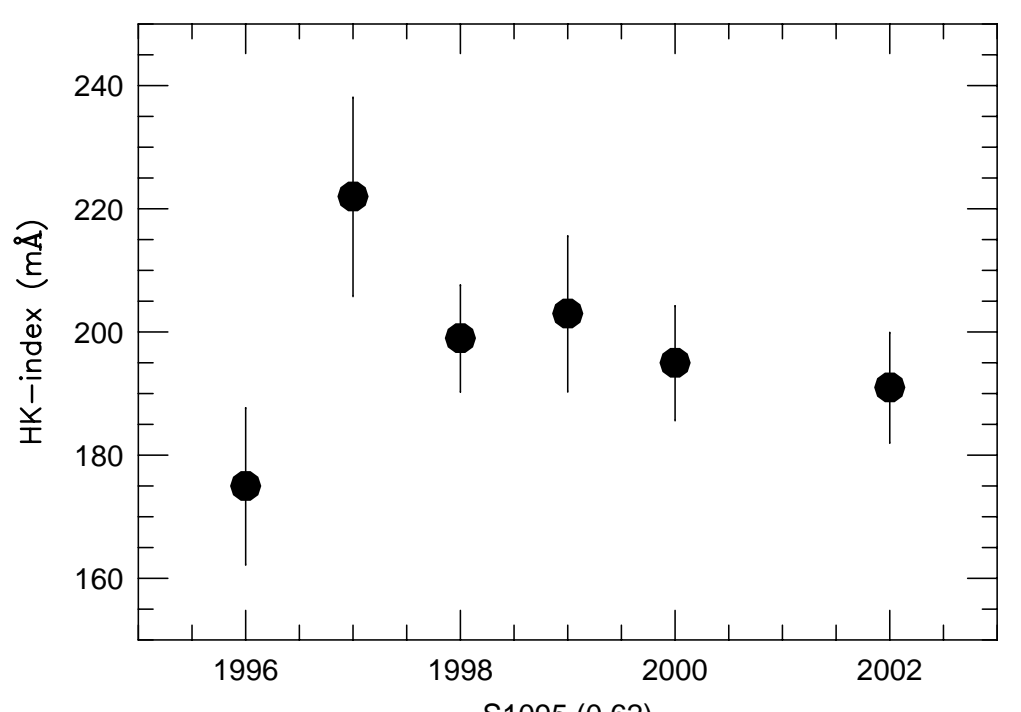

S1095 (0.62)

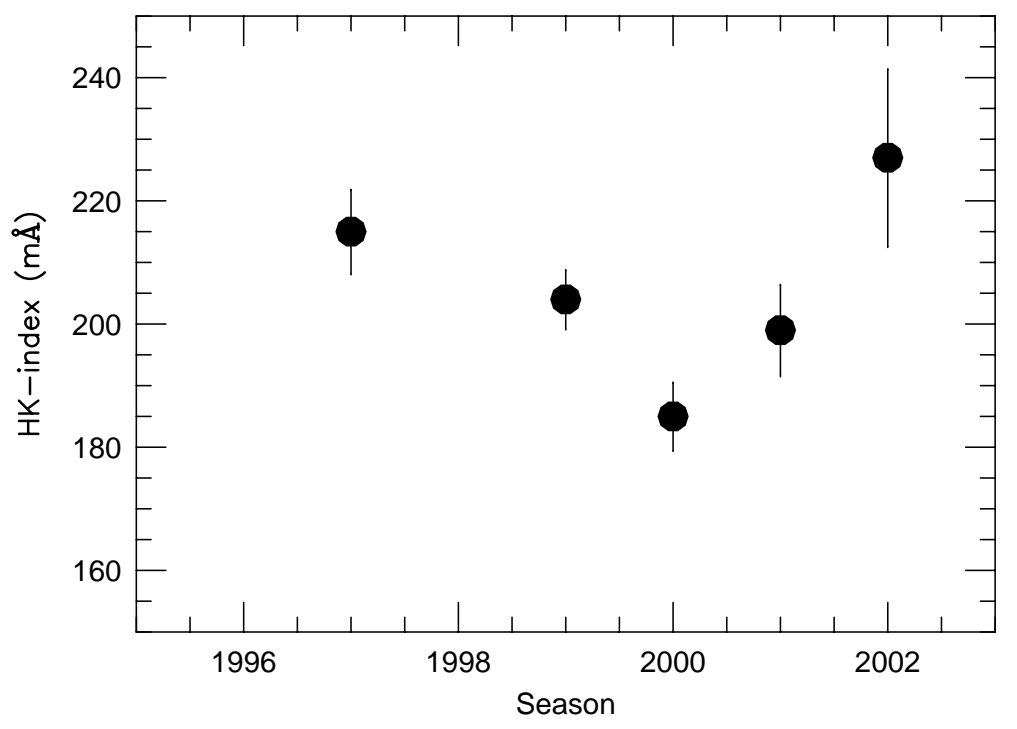

S945 (0.62)
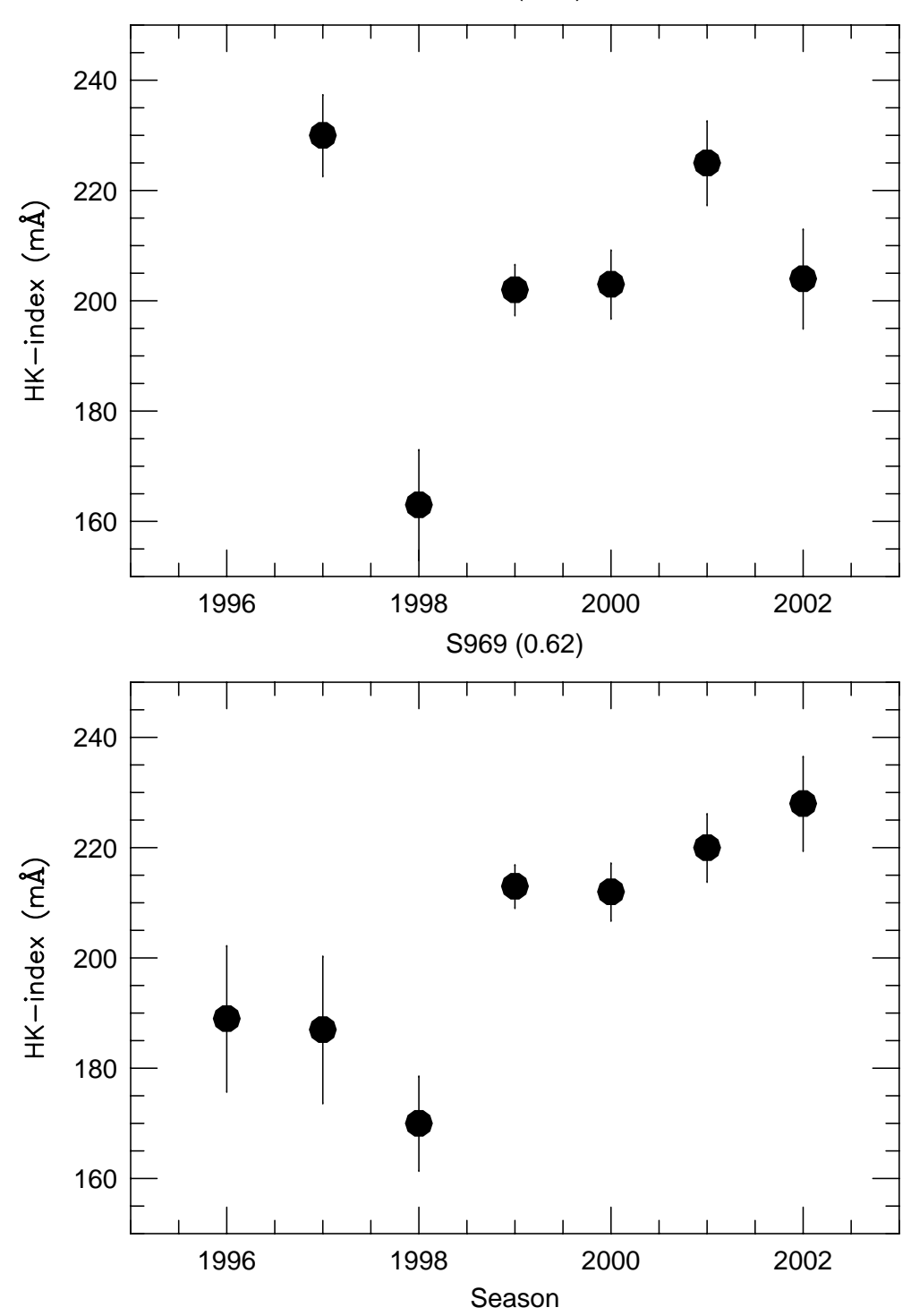
S1057 (0.62)
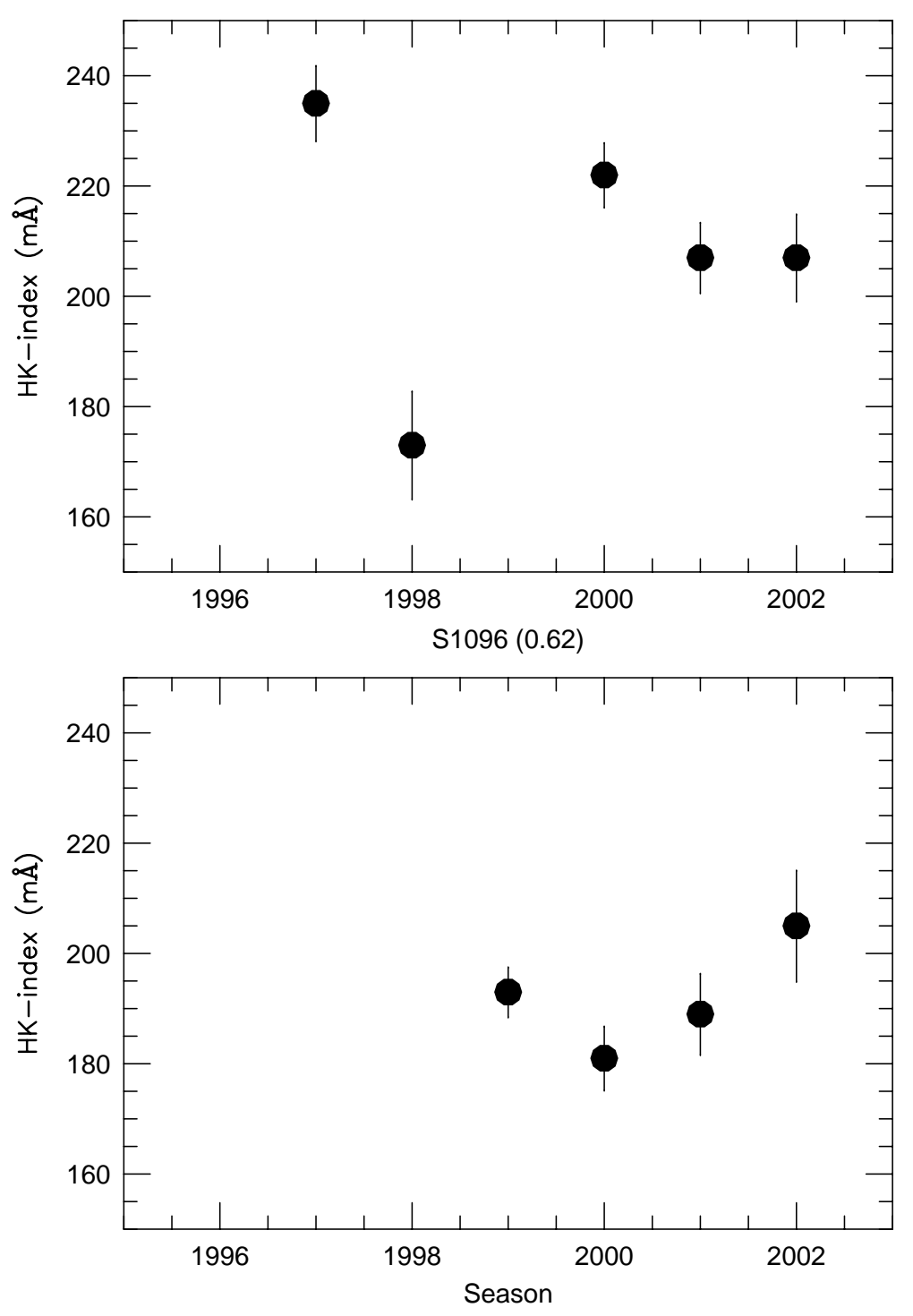

S1089 (0.62)
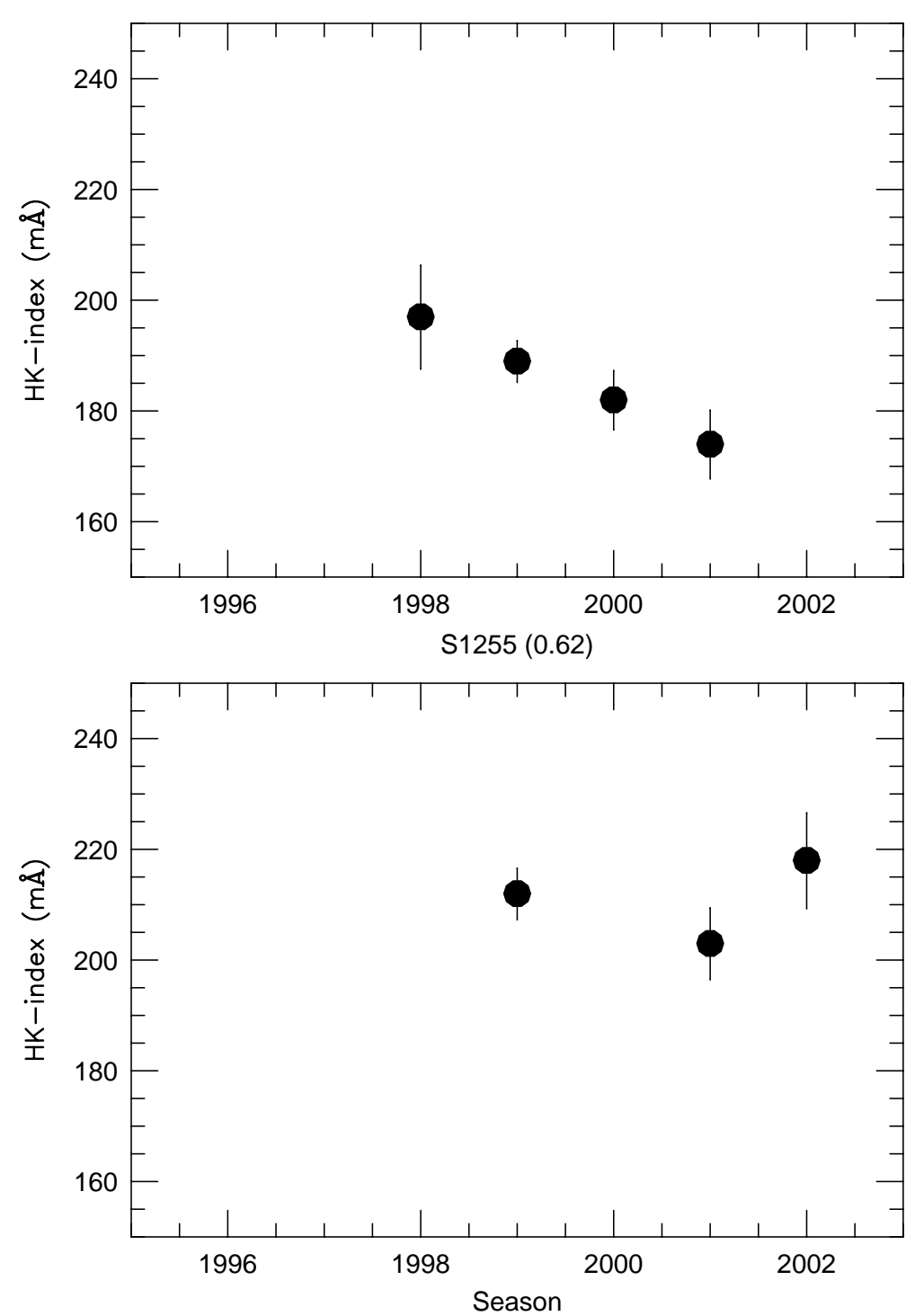
S1341 (0.62)
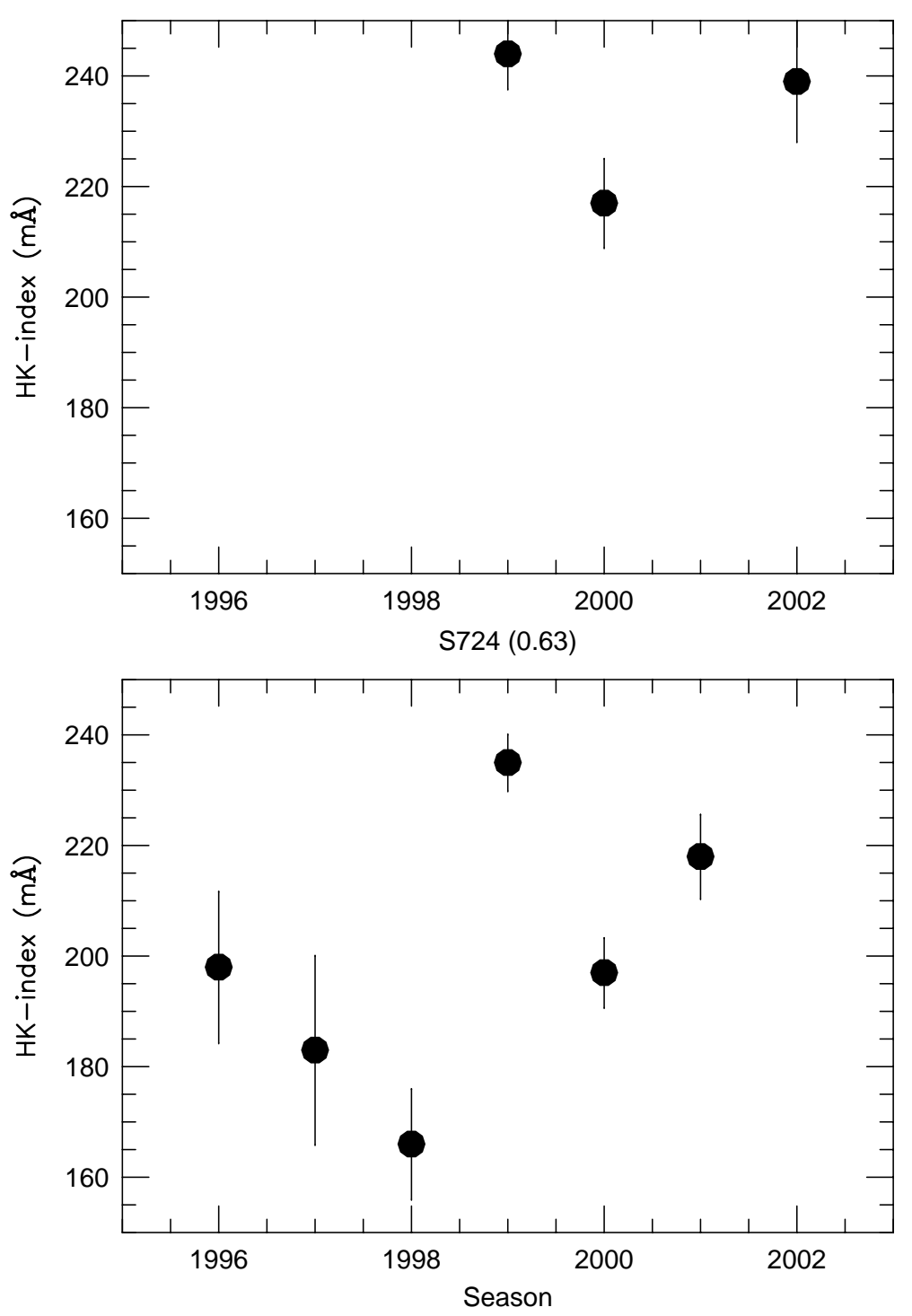

S1452 (0.62)
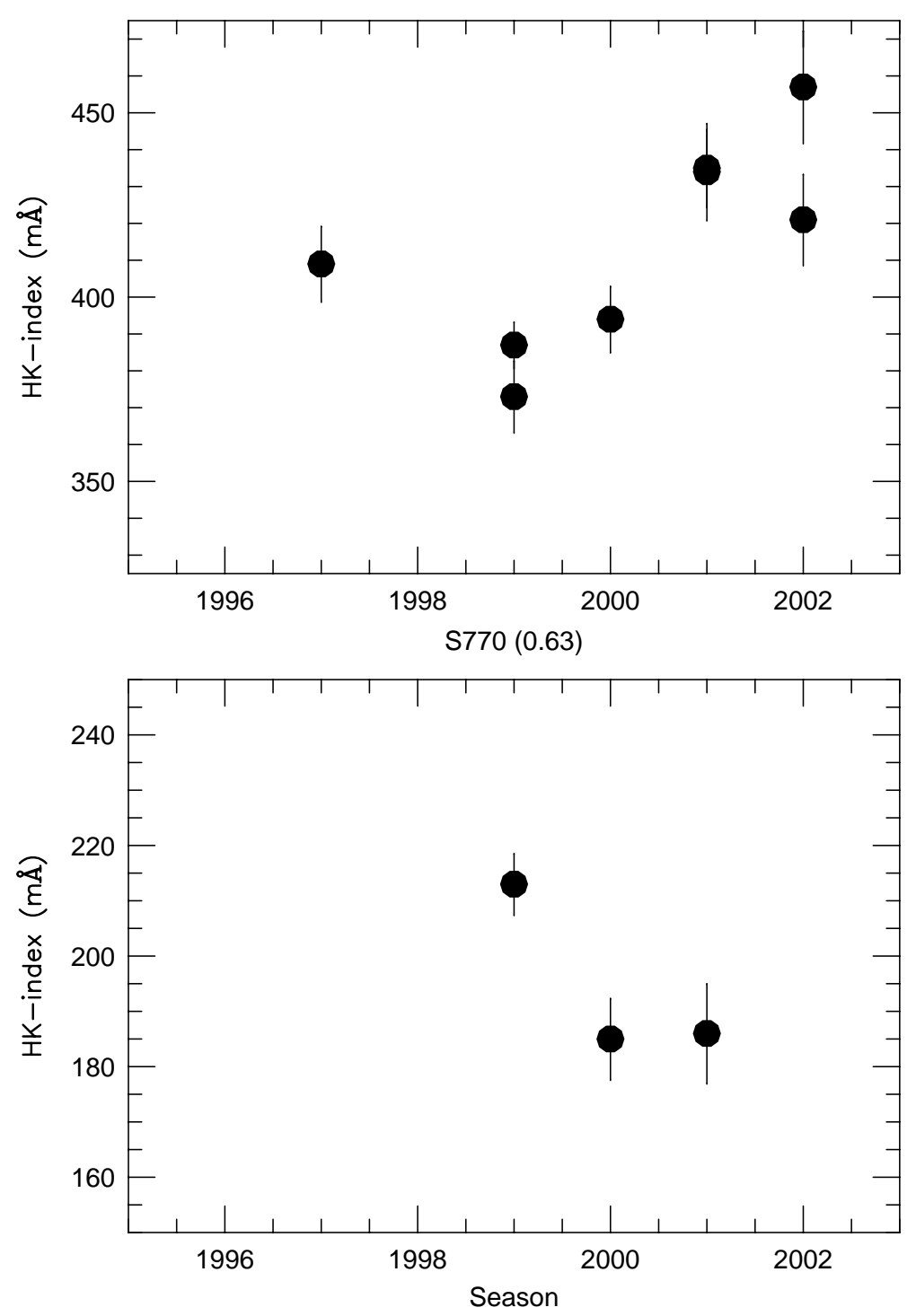
S777 (0.63)
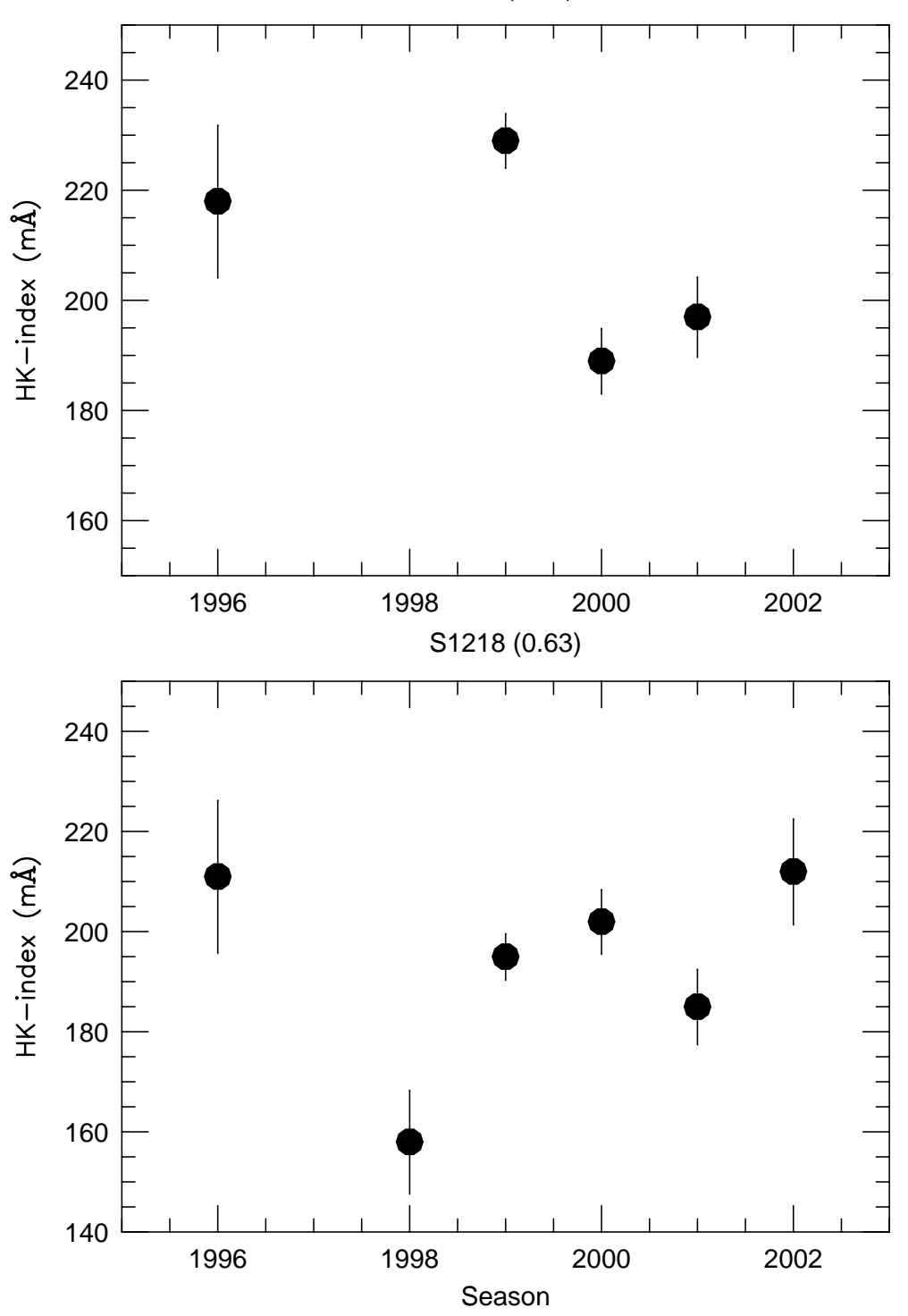

S991 (0.63)
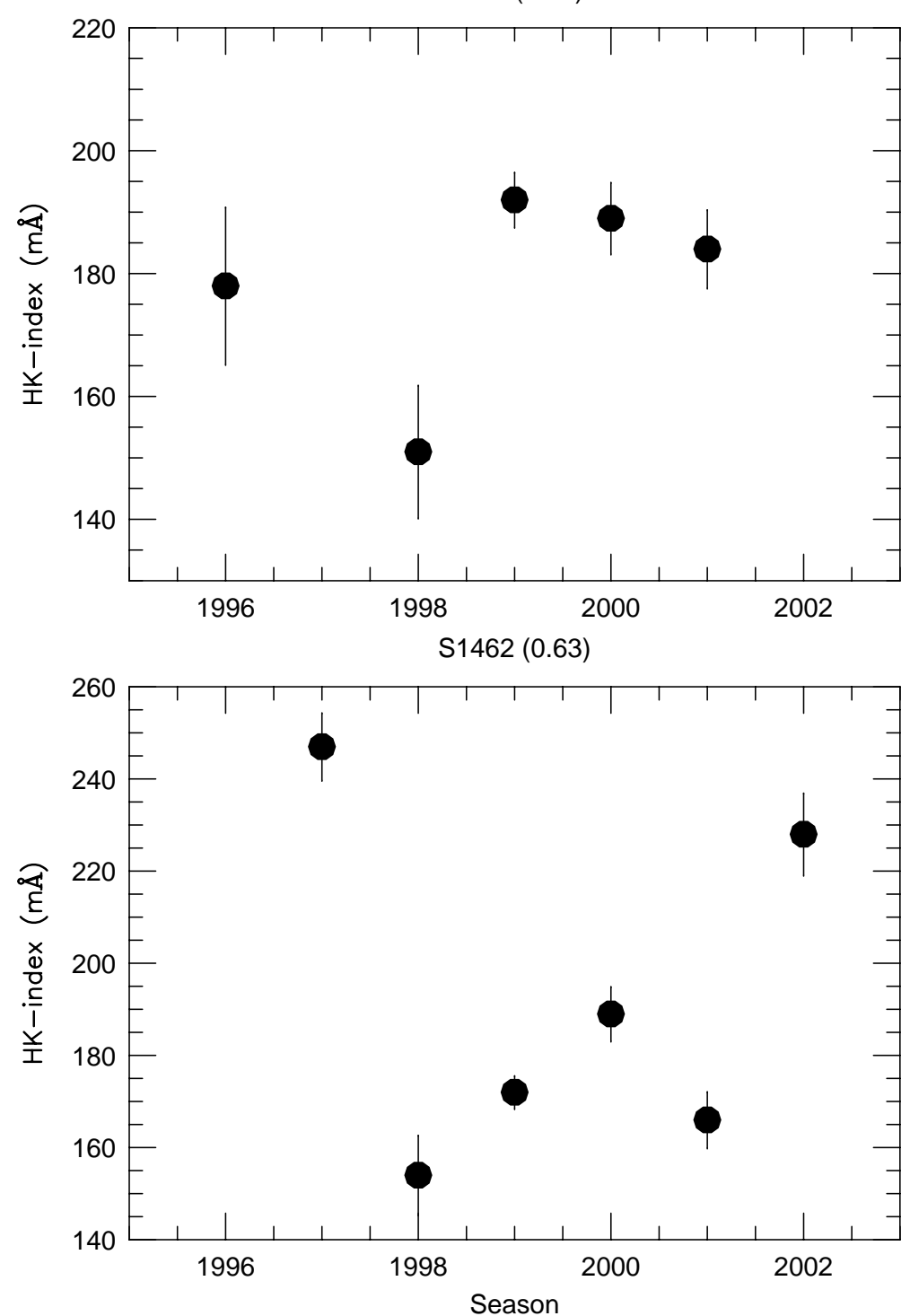
S1048 (0.64)
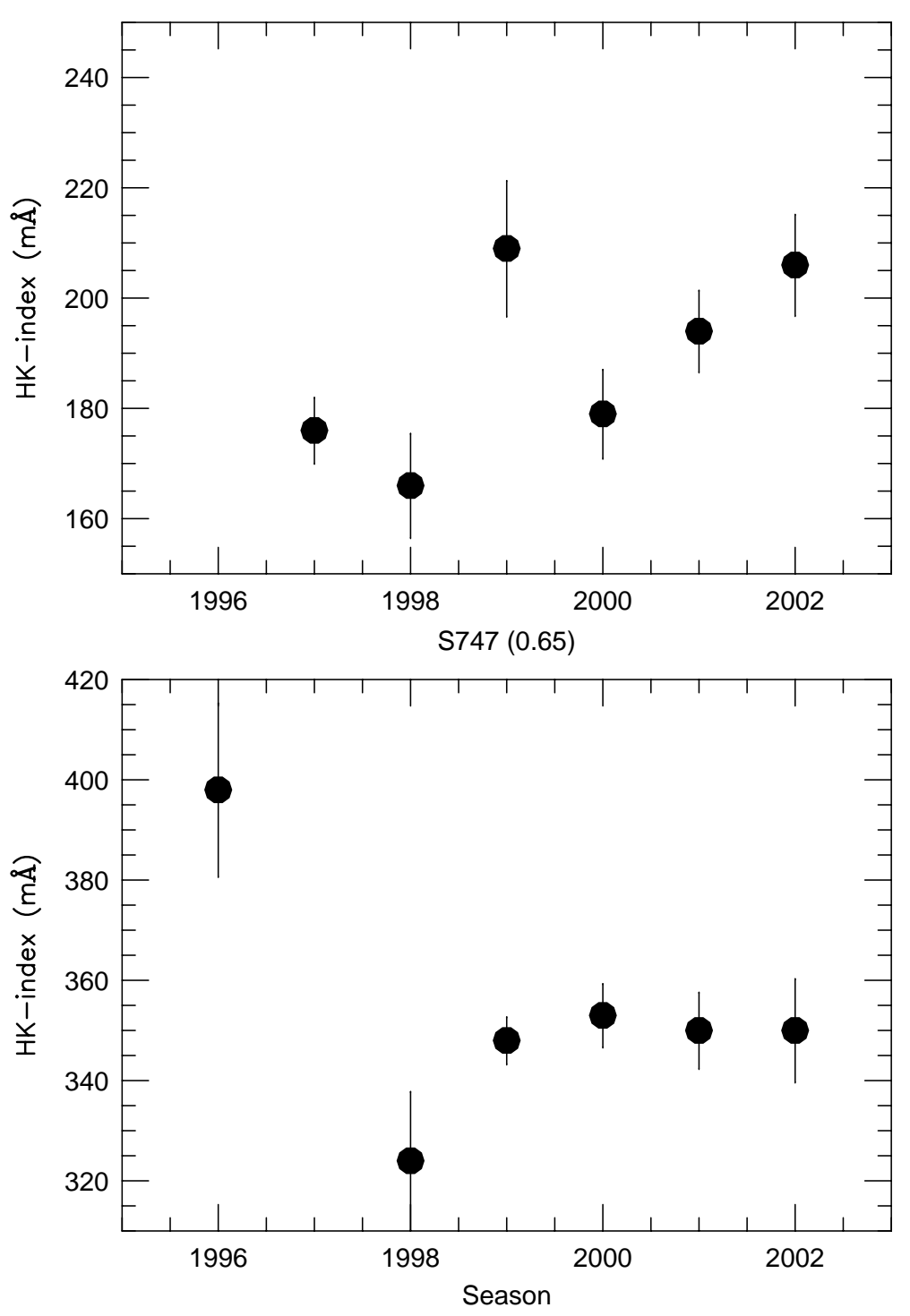

S1246 (0.64)

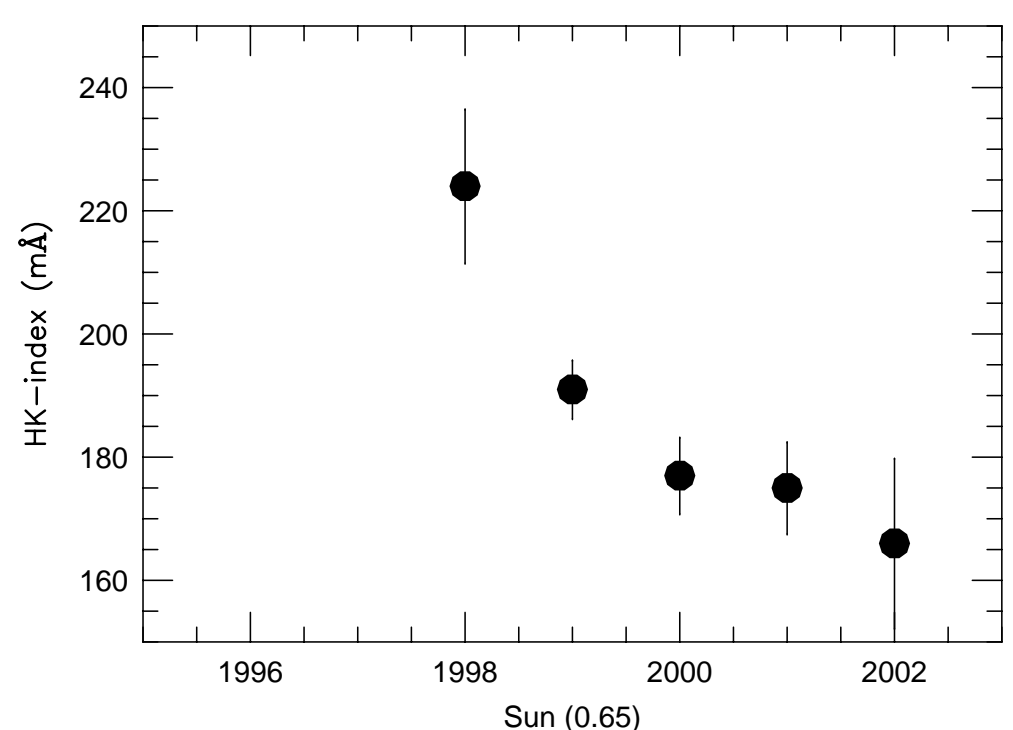

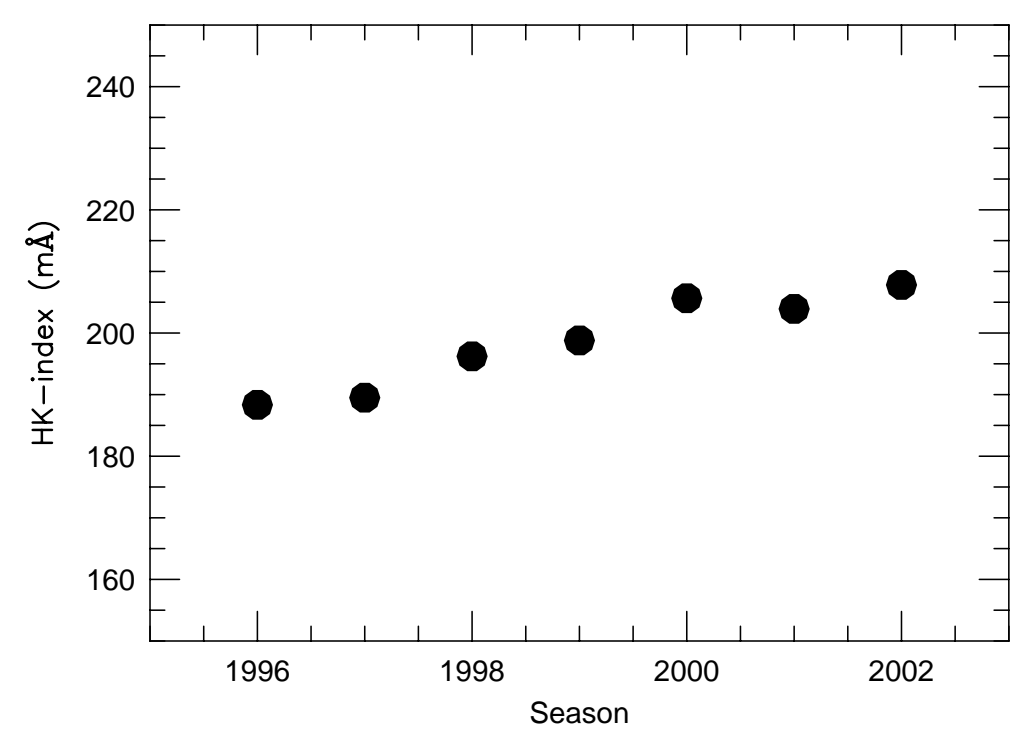


S785 (0.65)
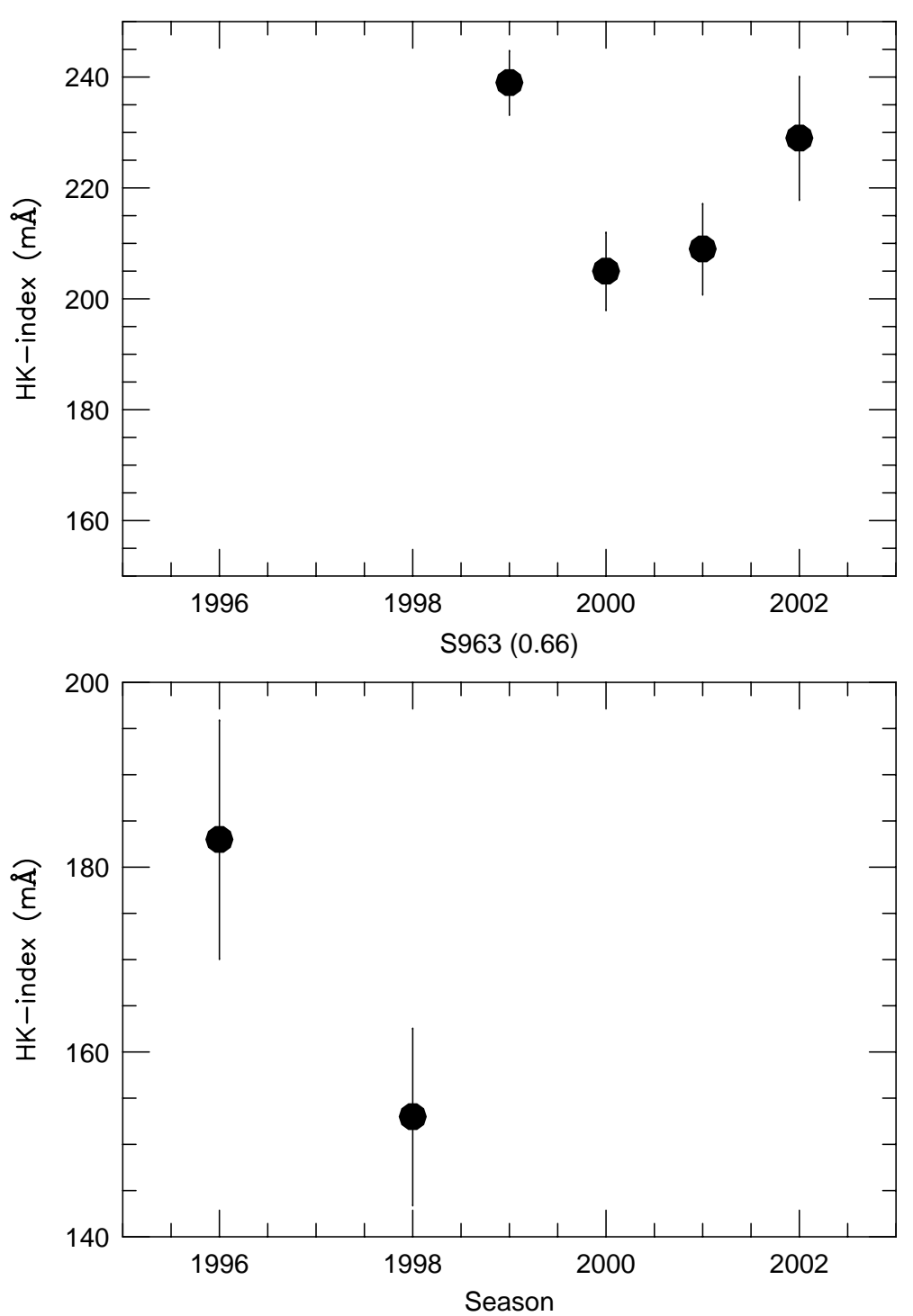

S746 (0.66)
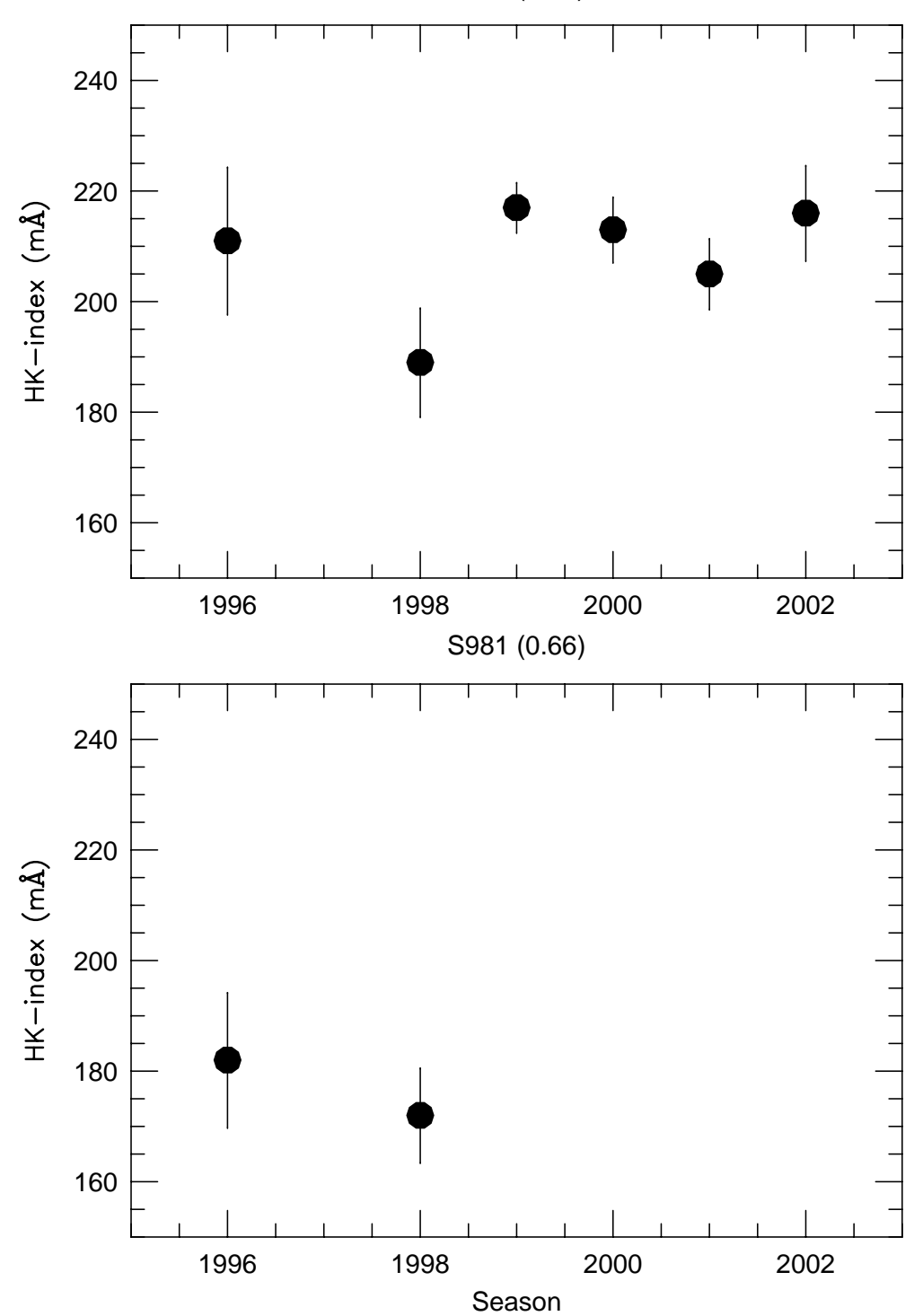
S1014 (0.66)
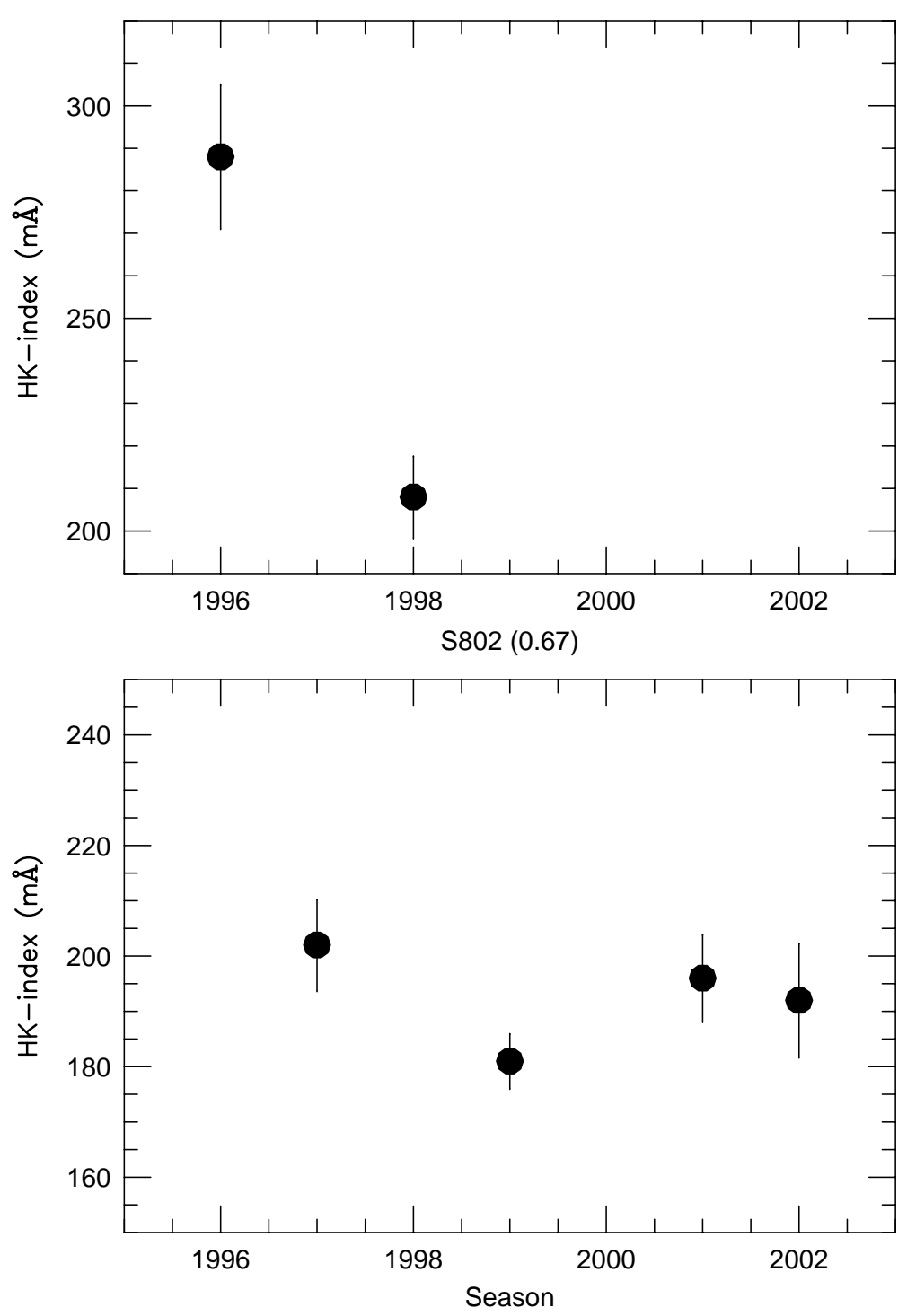

S801 (0.67)
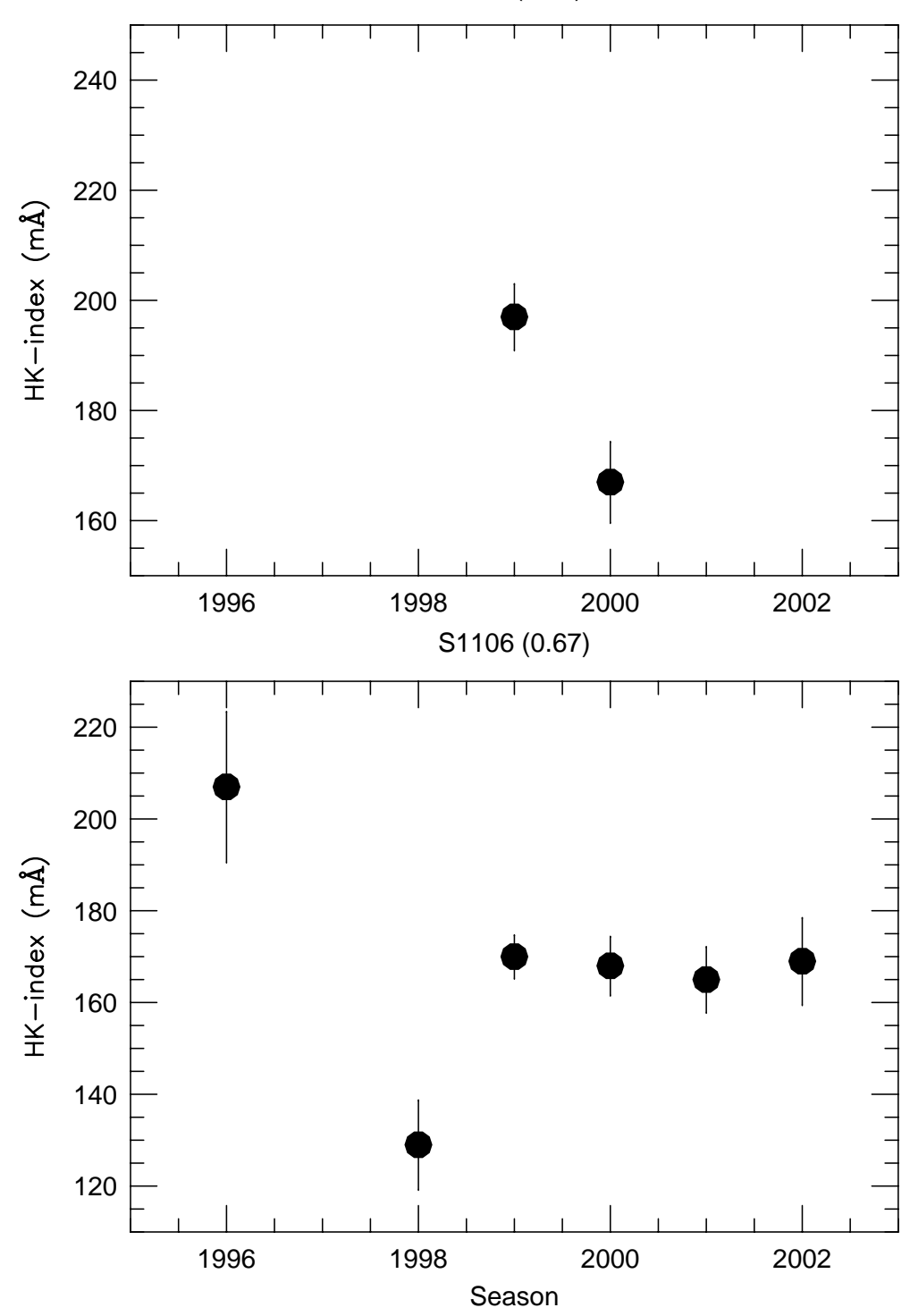
S1203 (0.67)
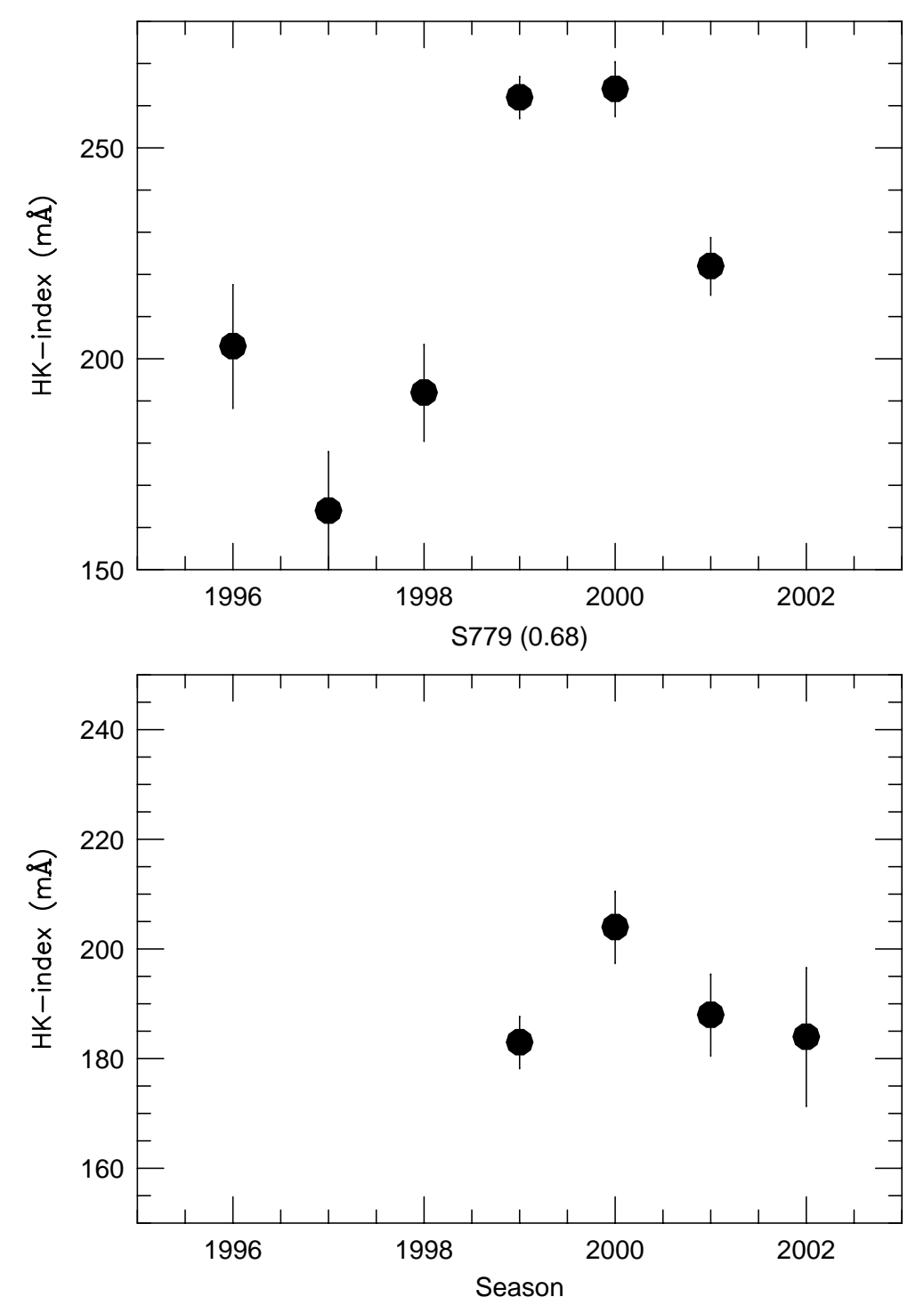

S1477 (0.67)
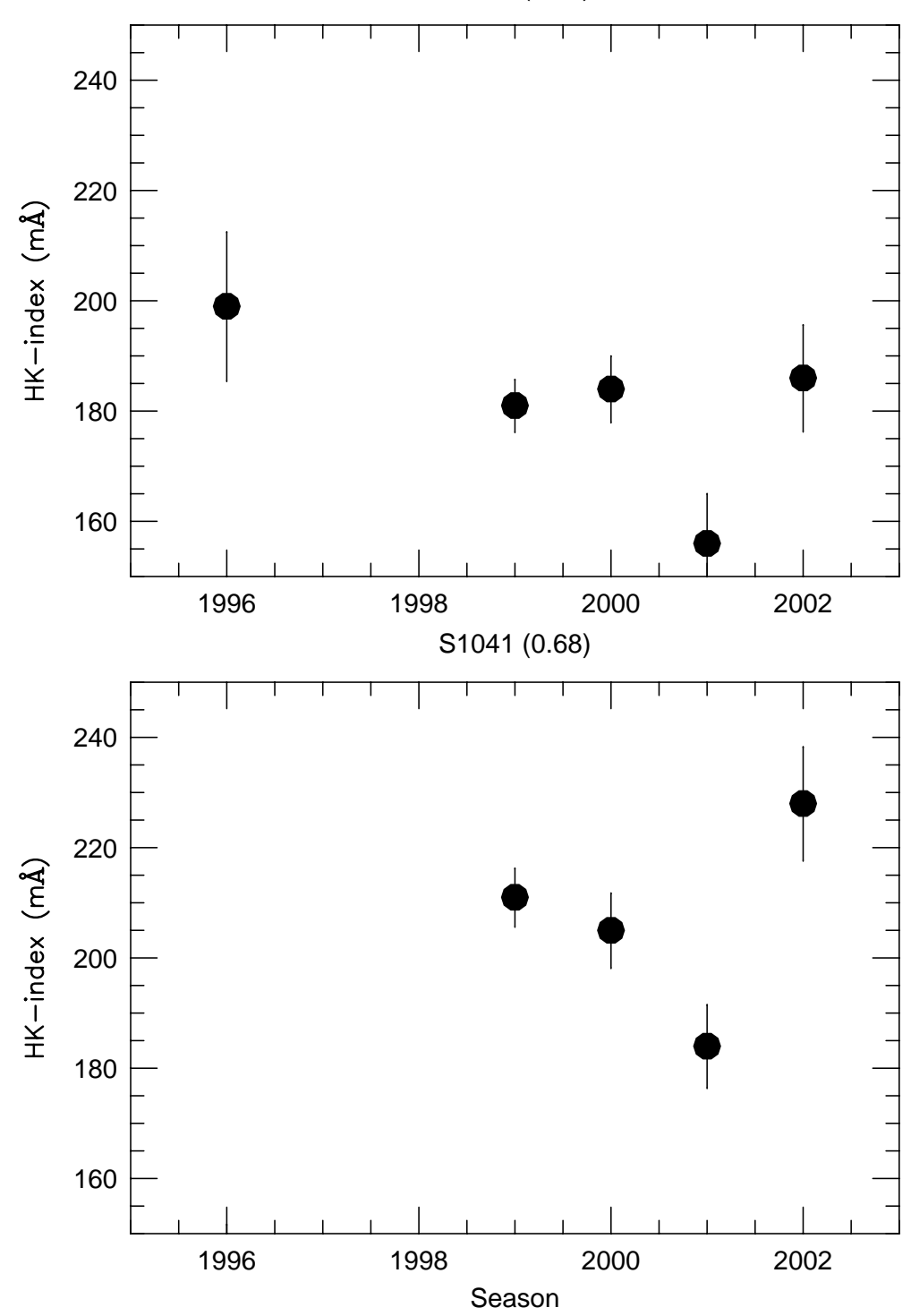
S1068 (0.70)

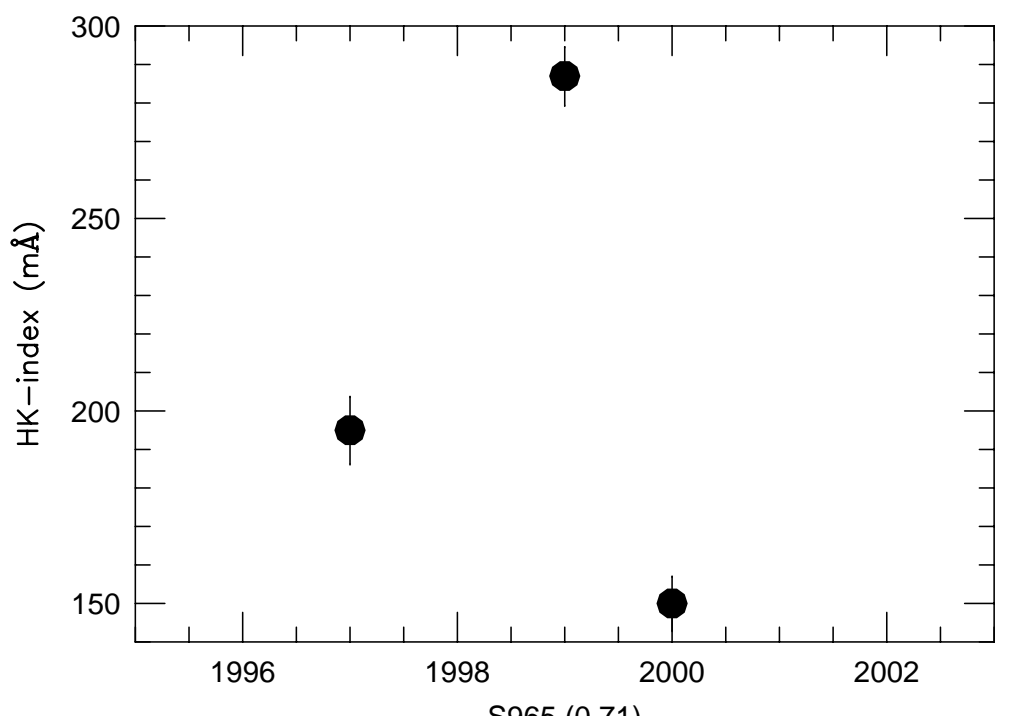

S965 (0.71)

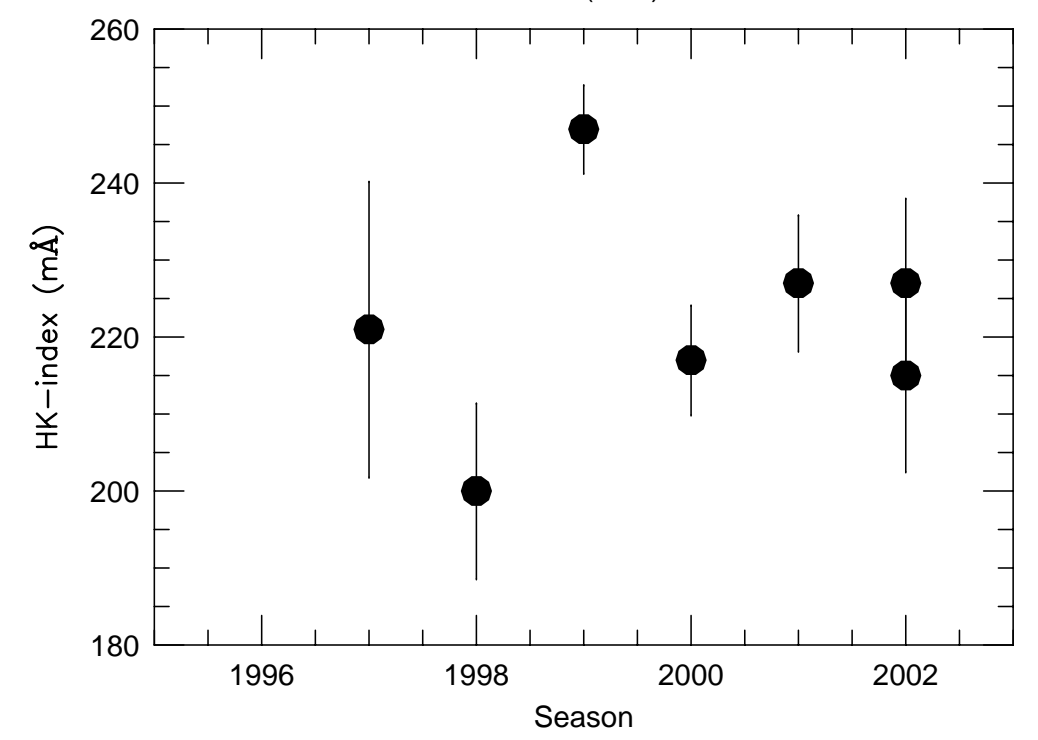

S1251 (0.70)

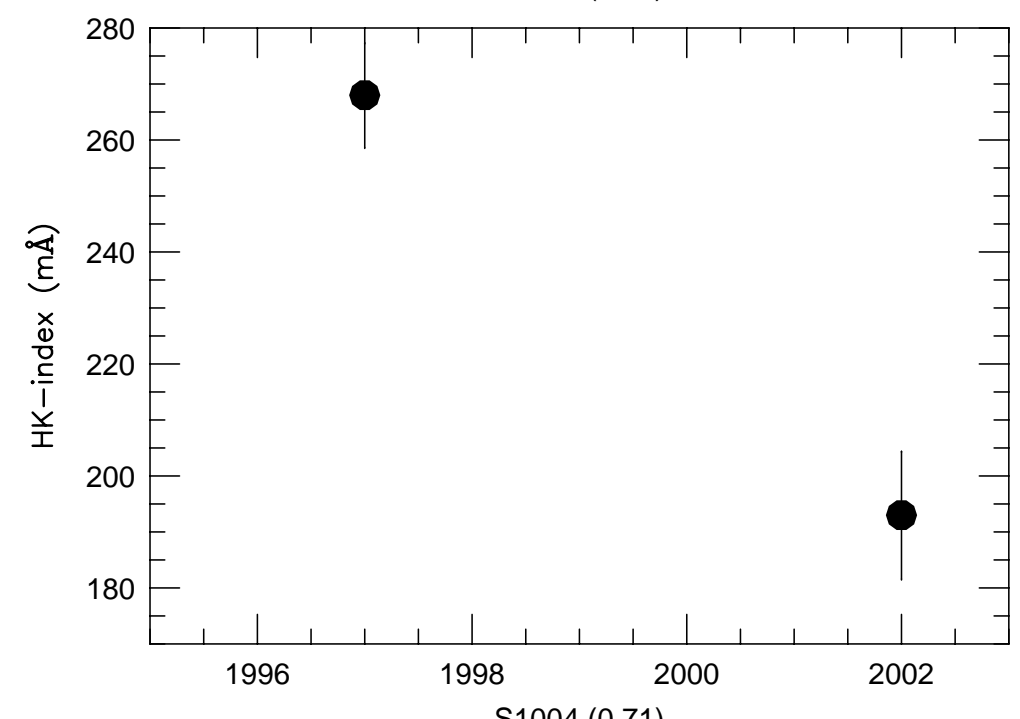

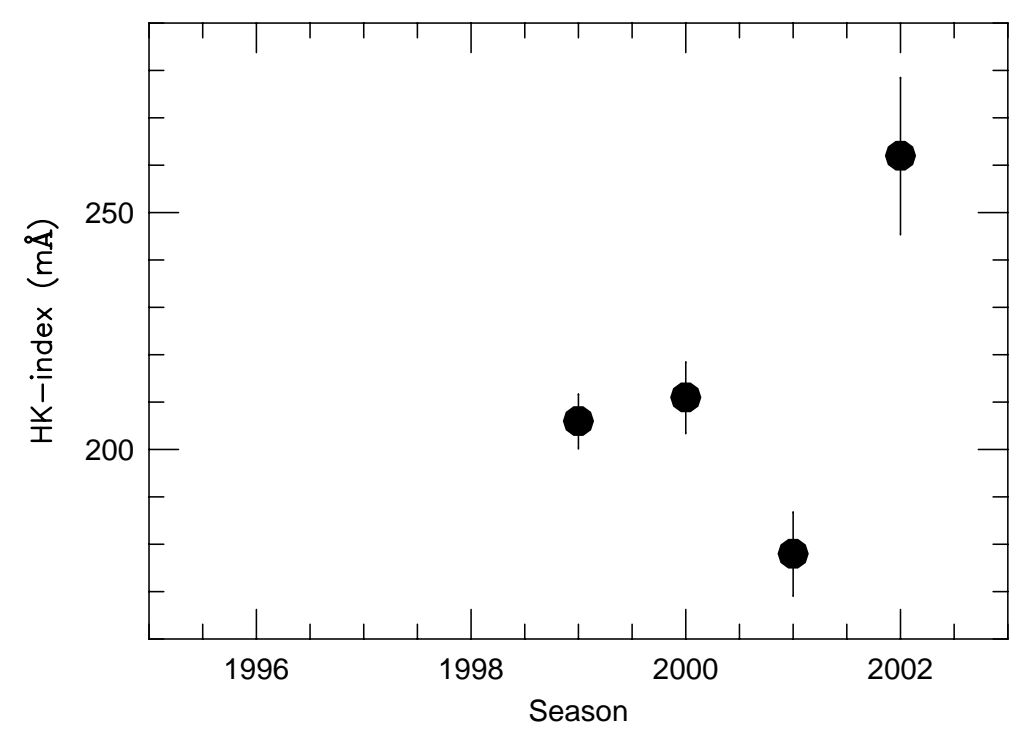


S1269 (0.71)

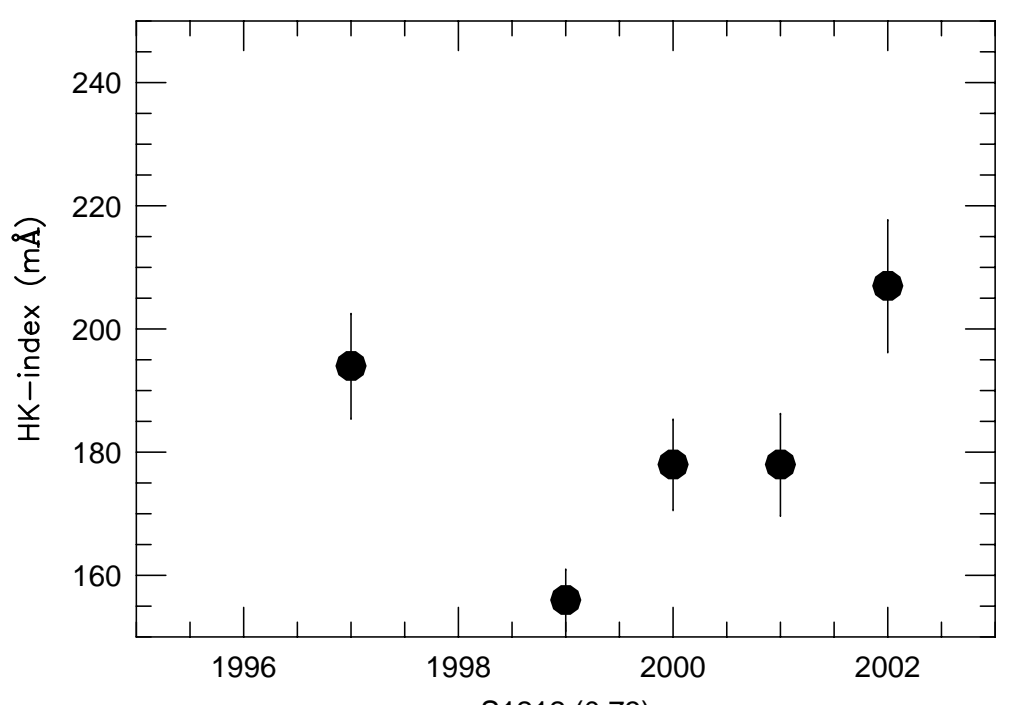

$\mathrm{S} 1212(0.73)$

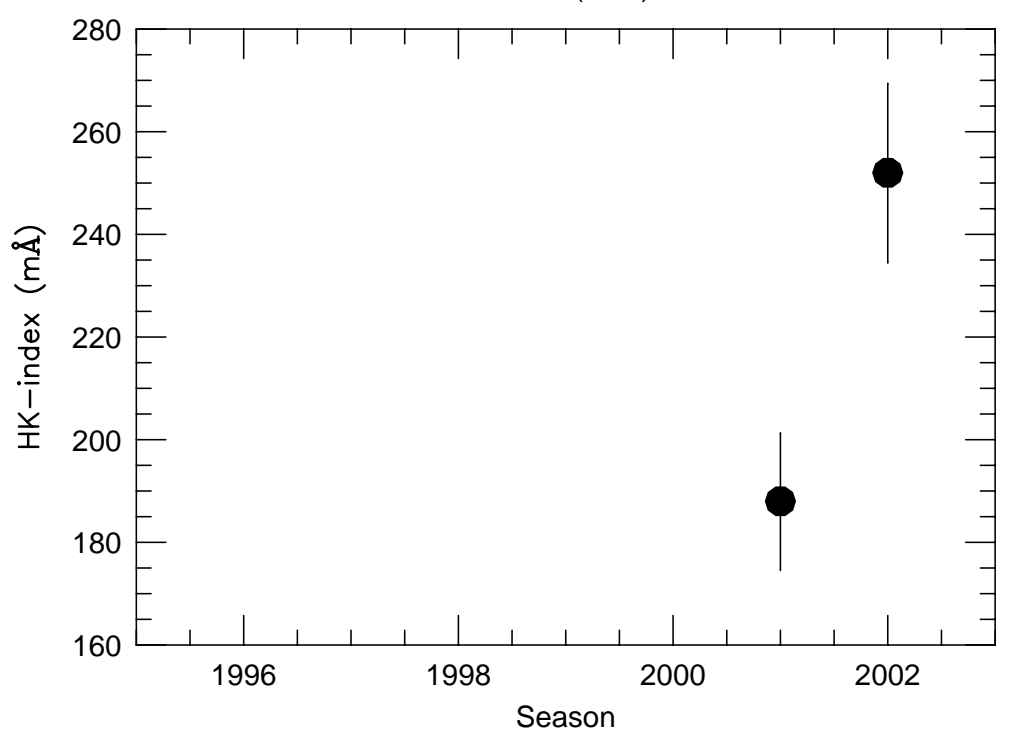

S1289 (0.71)
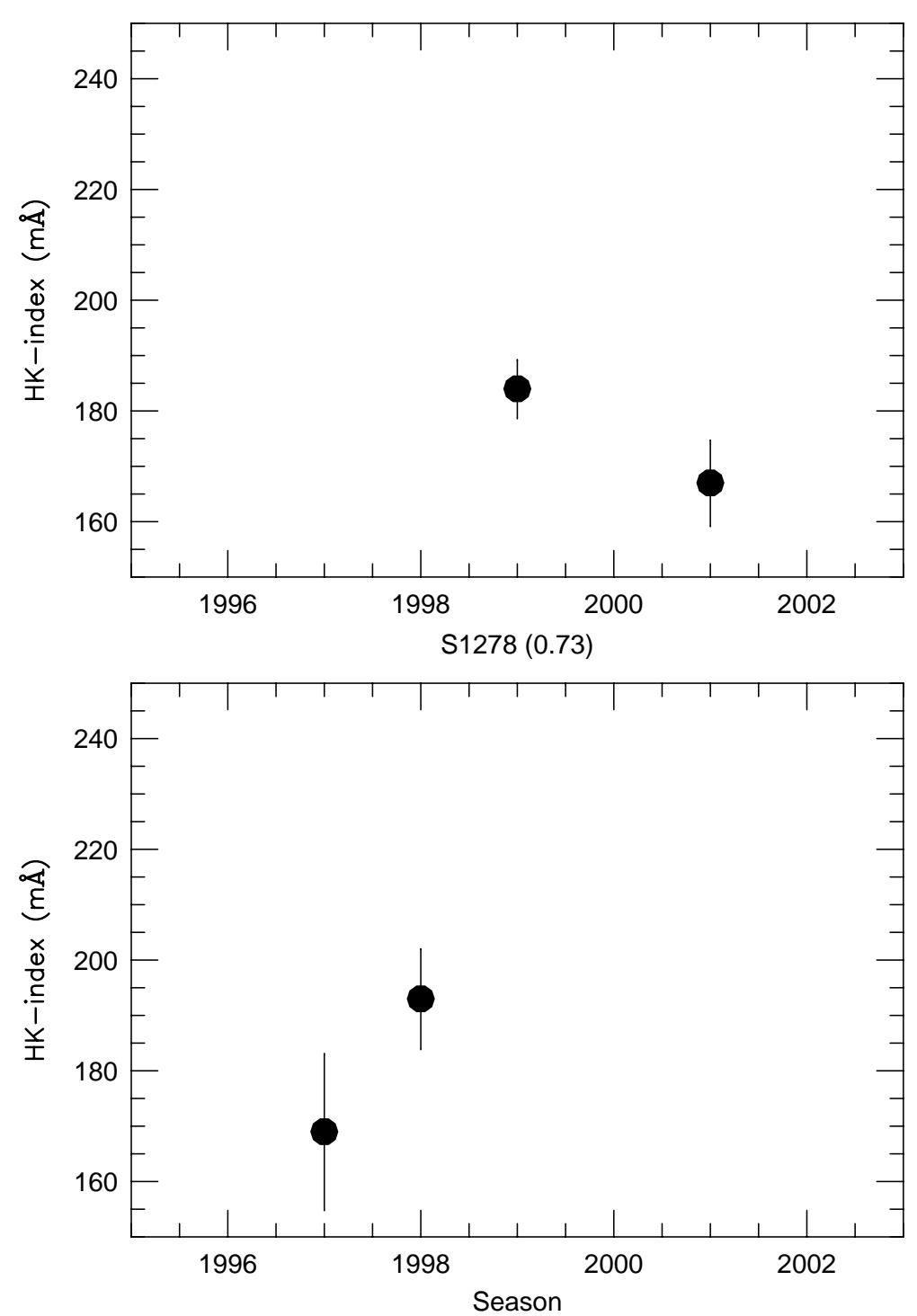
S1473 (0.73)
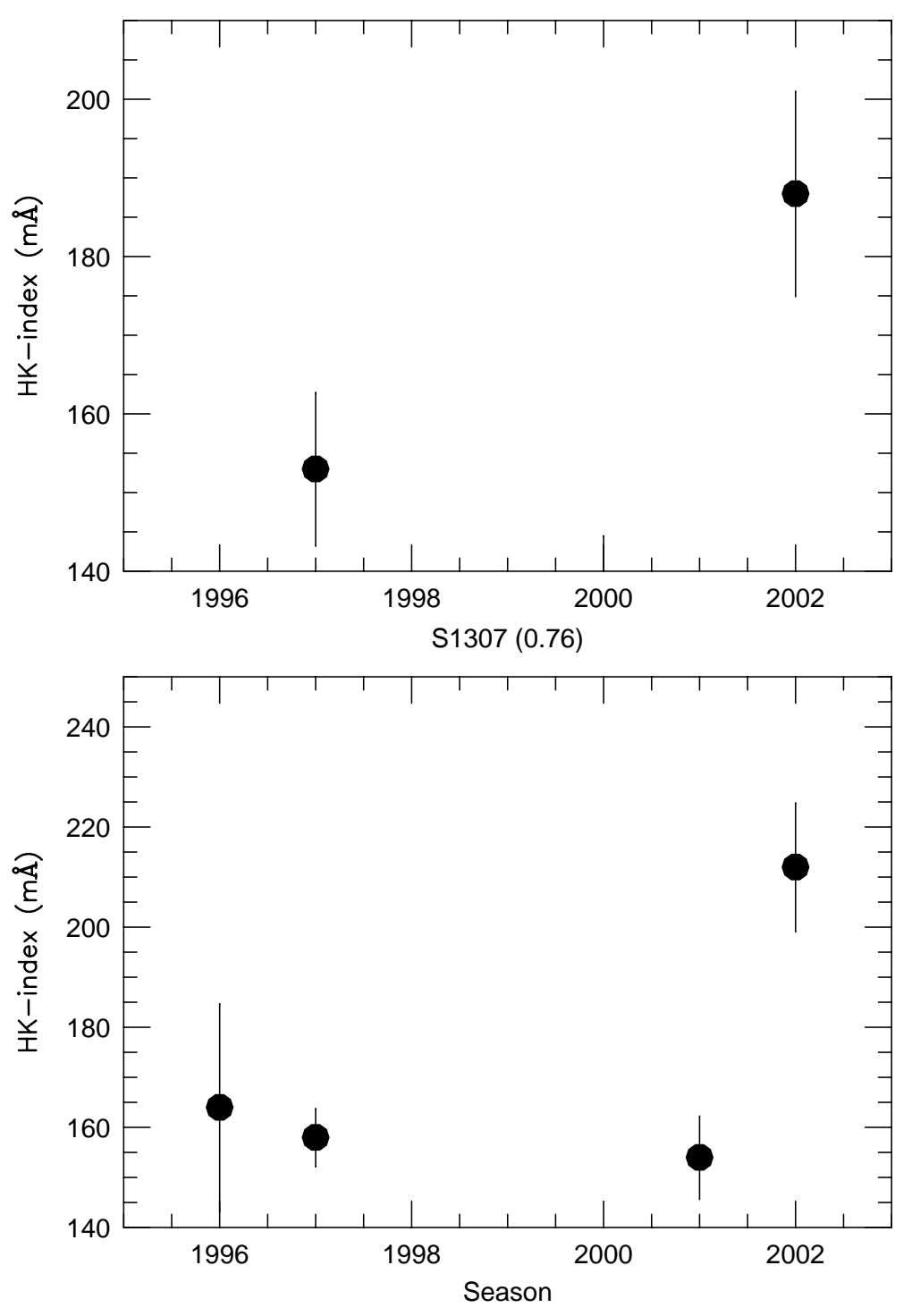

S1249 (0.74)

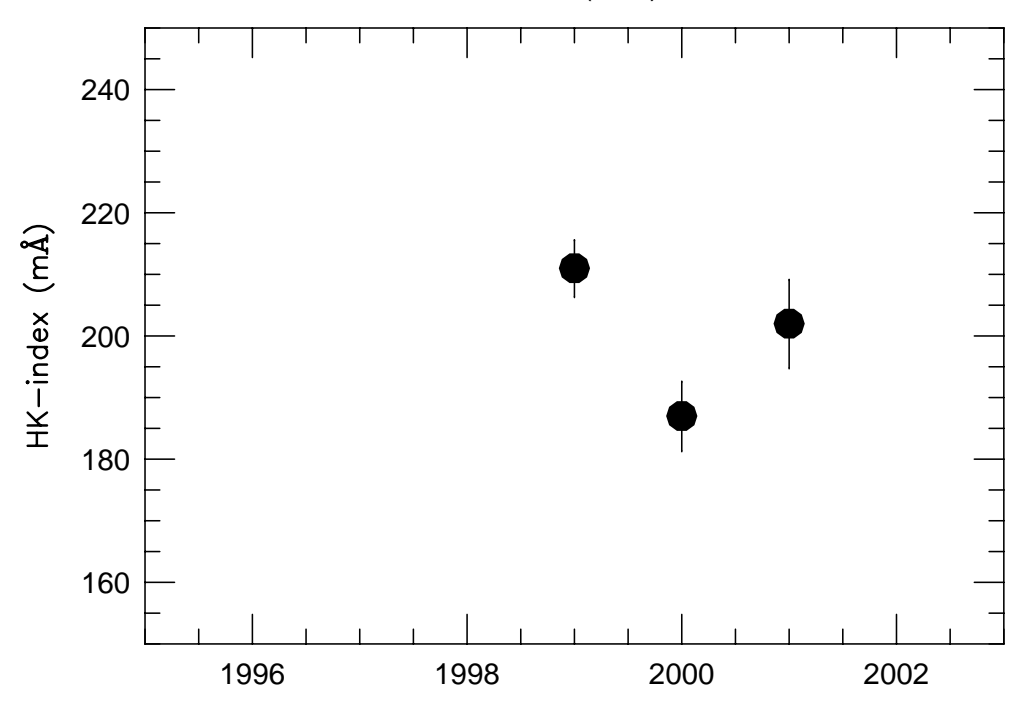


Sun (0.65)

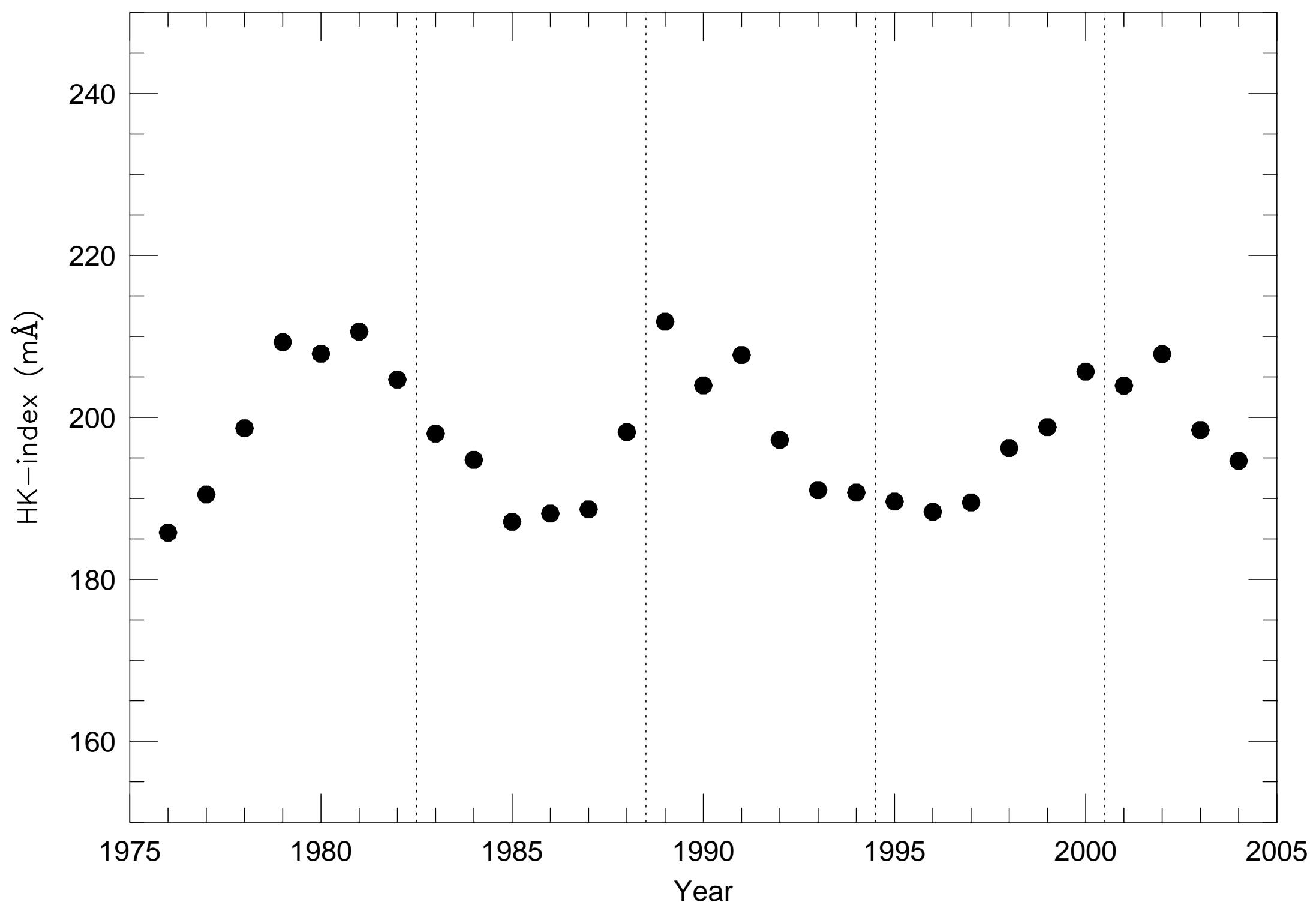




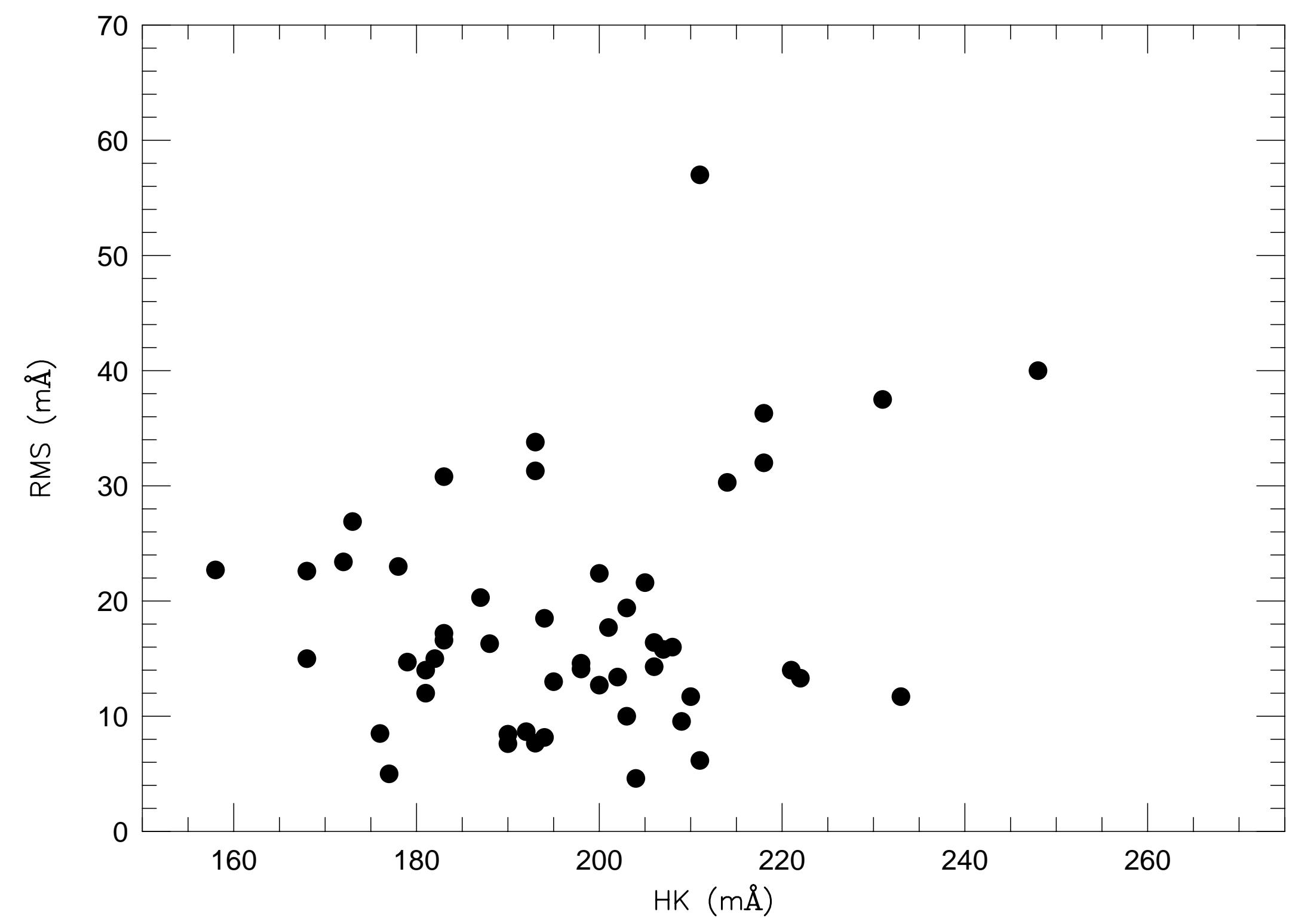




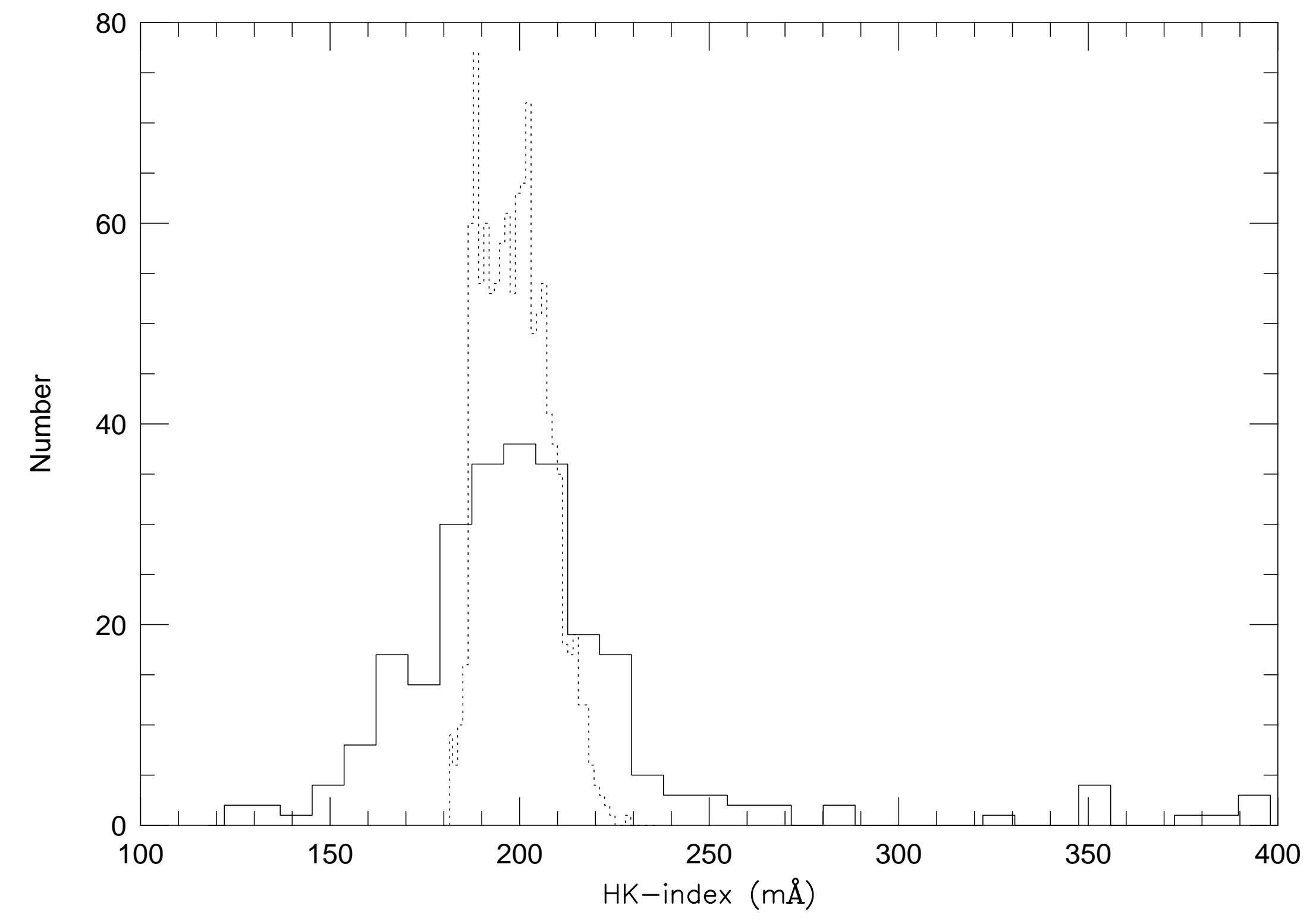




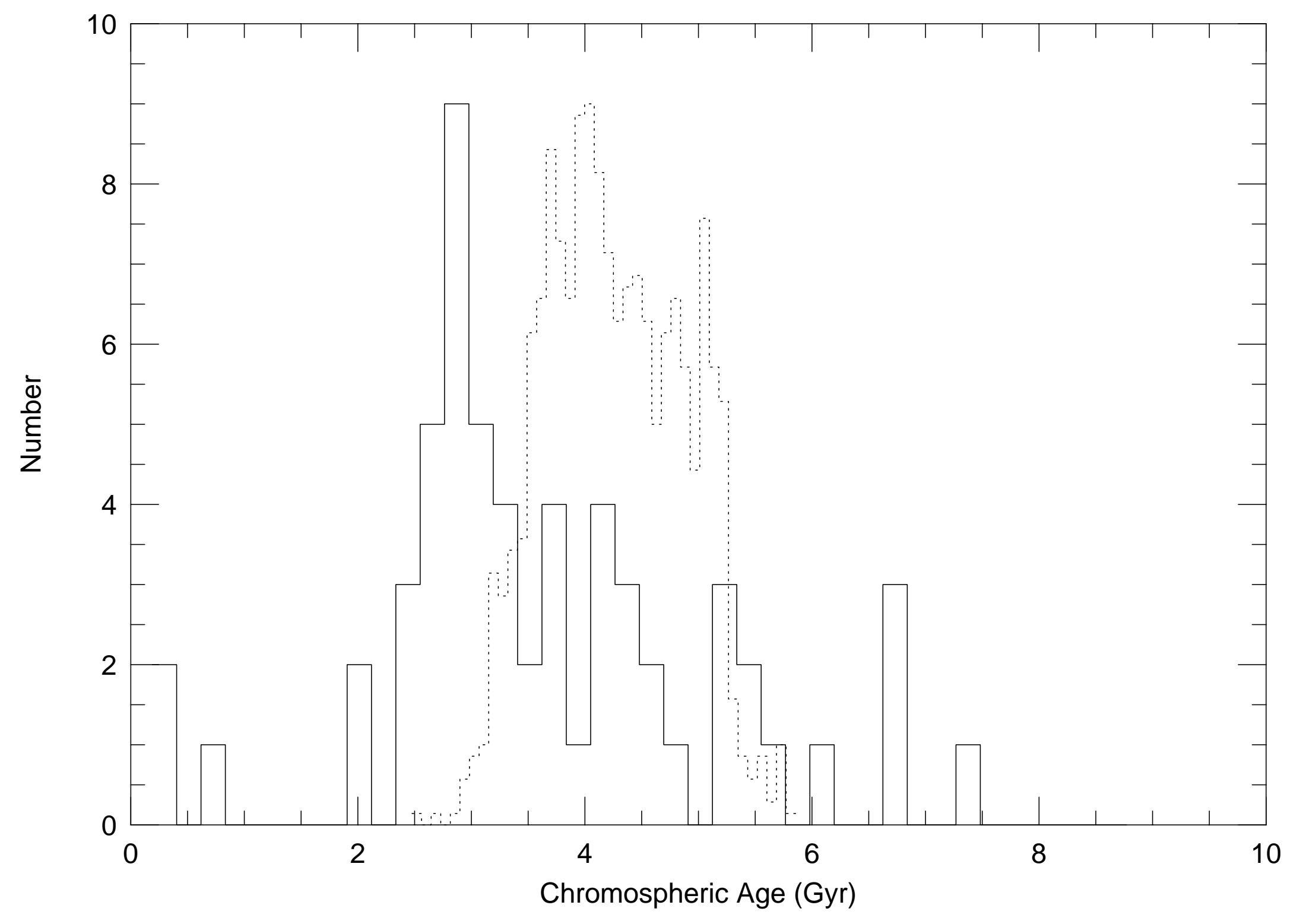




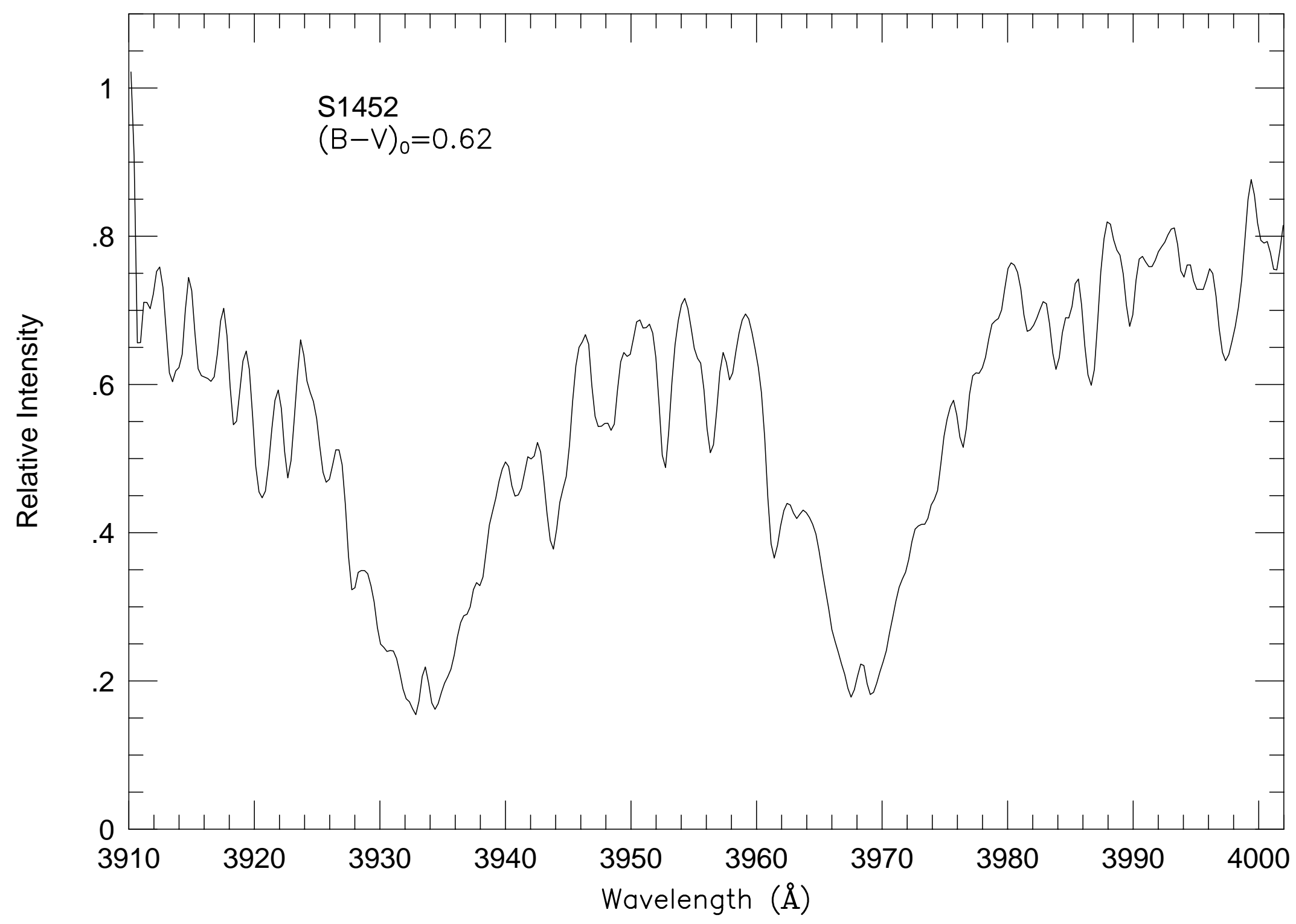

